\title{
REGULATION OF LYSOSOME BIOGENESIS BY PHOSPHOINOSITIDES AND PHAGOCYTOSIS
}

\author{
By \\ Christopher Choy \\ B.Sc., Ryerson University, 2012 \\ A dissertation \\ presented at Ryerson University \\ in partial fulfillment of the \\ requirements for the degree of \\ Doctor of Philosophy \\ in the program of \\ Molecular Science
}

Toronto, Ontario, Canada, 2018

(OChristopher Choy, 2018 


\section{AUTHOR'S DECLARATION FOR ELECTRONIC SUBMISSION OF A DISSERTATION}

I hereby declare that I am the sole author of this dissertation. This is a true copy of the dissertation, including any required final revisions, as accepted by my examiners.

I authorize Ryerson University to lend this dissertation to other institutions or individuals for the purpose of scholarly research

I further authorize Ryerson University to reproduce this dissertation by photocopying or by other means, in total or in part, at the request of other institutions or individuals for the purpose of scholarly research.

I understand that my dissertation may be made electronically available to the public. 


\title{
REGULATION OF LYSOSOME BIOGENESIS BY \\ PHOSPHOINOSITIDES AND PHAGOCYTOSIS
}

\author{
Christopher Choy \\ Doctor of Philosophy, 2018 \\ Molecular Science, Ryerson University
}

\begin{abstract}
Lysosomes are acidic organelles responsible for molecular degradation, energy balance, and pathogen clearance. Consequently, lysosome dysfunction is linked to numerous diseases, including lysosome storage diseases. Notably, enhancing lysosome biogenesis ameliorates cell function and helps clear metabolites. The transcription factor EB (TFEB) is a master regulator of lysosome biogenesis, and thus a potential therapeutic target. Among known regulators of TFEB, the mammalian target of rapamycin complex 1 (mTORC1) is best understood. In nutrient-rich cells, mTORC1 is activated and represses TFEB by phosphorylation. Upon starvation, mTORC1 is inactivated and TFEB enters the nucleus, upregulating lysosomal gene expression to enhance cellular degradation for energy recovery. Numerous other TFEB-dependent pathways have been identified. We aim to understand how TFEB is regulated in two additional contexts: in lysosome enlargement during phosphatidylinositol 3,5-bisphosphate $\left[\operatorname{Ptd} \operatorname{Ins}(3,5) P_{2}\right]$ depletion and in phagocytosis.
\end{abstract}

First, $\operatorname{PtdIns}(3,5) P_{2}$ is required for maintaining lysosome size by an incompletely understood mechanism. We hypothesized that TFEB-mediated lysosome biogenesis contributes de novo lysosomal material. Acute depletion of PtdIns(3,5)P $P_{2}$-synthesizing 
kinase PIKfyve induced TFEB nuclear accumulation. Despite increases in transcription, little to no protein translation was observed. Furthermore, $t \mathrm{fe}^{-1-}$ cells and cells blocked with cycloheximide were similar to wild-type cells, with regard to the number and size of lysosomes during PIKfyve inhibition cells, suggesting biosynthesis is not necessary for lysosome enlargement. However, TFEB still becomes active by an known mechanism. We show that TFEB nuclear localization during PIKfyve inhibition was not due to mTORC1 inactivation but may result from GSK3 inhibition.

Secondly, phagocytosis allows immune cells to sequester potential pathogens by engulfing them into phagosomes. These phagosomes are then degraded by the lysosome. We postulated that phagocytosis would enhance TFEB-mediated lysosome biogenesis to promote pathogen killing. Fc $\gamma$ receptor-mediated phagocytosis activated TFEB and increased biosynthesis of select lysosomal genes, augmenting existing lysosomes and enhancing proteolysis. To understand how TFEB was activated by the Fc $\gamma$ receptor, we inhibited key signaling and trafficking mediators. Particle internalization, phagosome formation, and phagosome maturation appear to be necessary for TFEB activation.

Overall, our work uncovers two additional mechanisms that may govern TFEBactivation. 


\section{Acknowledgements}

First, I acknowledge my supervisor and mentor, Dr. Roberto Botelho, whose guidance and support has been instrumental in my personal and professional growth. Thank you for taking a chance on me when I had no experience. Thank you for being patient with me and providing me with tough love that drove me to be my best.

I would also like to acknowledge my graduate committee: Dr. Warren Wakarchuk and Dr. Jeffrey Fillingham for challenging me to do better. Thank you for being a calm voice when I needed reassurance. In addition, I acknowledge Dr. Costin Antonescu who has provided additional mentorship during lab meetings and ad-hoc committees.

I am also grateful for the support provided by the Botelho and Antonescu lab family. Specifically, I thank Amra Saric, Shannon Ho, Grace Kim, and Monica Dayam for their roles in training me. I would also like to thank Dr. Matthew Gray, Golam Saffi, and all the volunteers and thesis students who have contributed to my thesis. Furthermore, I thank Victoria Hipolito, Krishna Chintaluri, Ralph Christian De Los Santos, Camilo Garay, Tracy Lackraj, and all those named above for making my time in graduate school enjoyable.

Finally, I thank my parents, my sister, and my partner, Cynthia Luong, for their unending love and support. 


\section{Table of Contents}

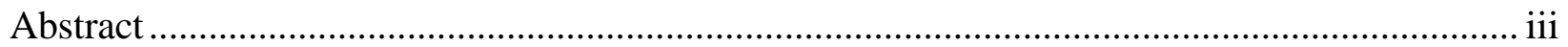

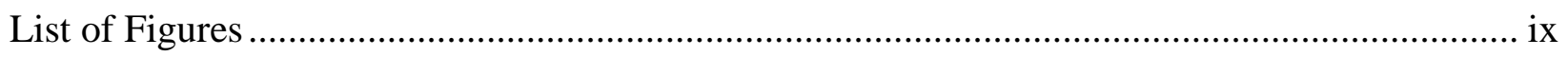

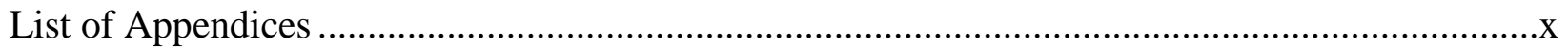

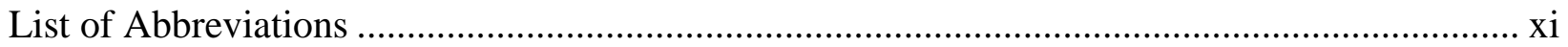

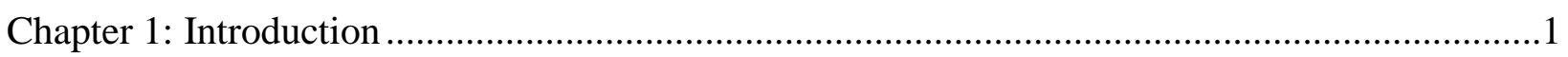

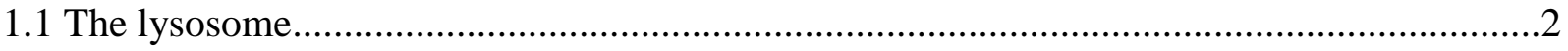

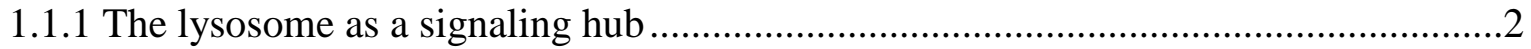

1.1.2 The MiT/TFE family of transcription factors ..................................................5

1.1.3 TFEB in autophagy and mTORC1 regulation.................................................6

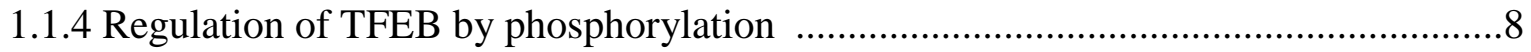

1.1.5 Regulation of TFEB by other post-translational modifications .............................10

1.2 Regulation of lysosome morphology by phosphatidylinositol 3,5-bisphosphate ..............12

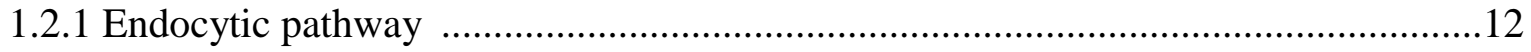

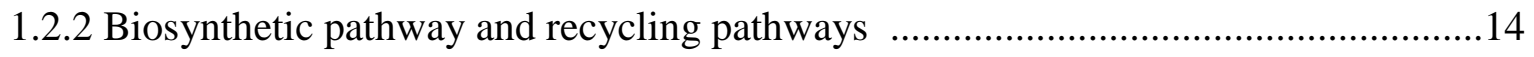

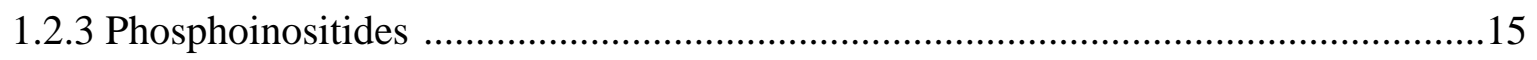

1.2.4 Phosphatidylinositol 3,5-bisphosphate ..............................................................17

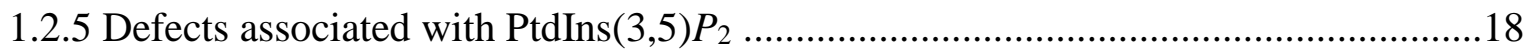

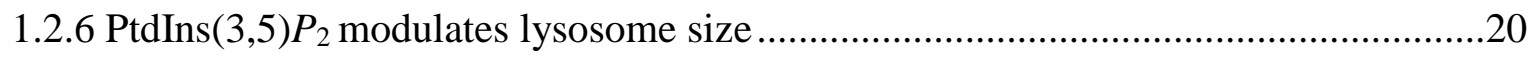

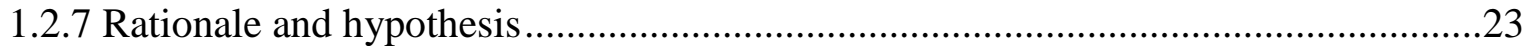

1.3 The regulation of lysosome biogenesis in phagocytosis ............................................24

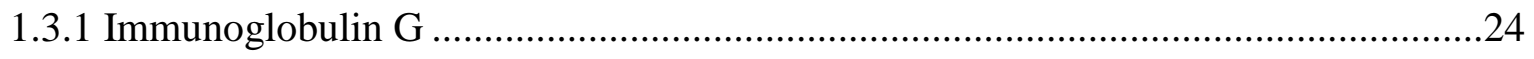

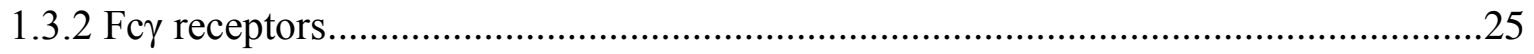

1.3.3 Phosphoinositide dynamics during phagocytosis ..............................................27

1.3.4 Actin polymerization via Rho GTPases .....................................................28

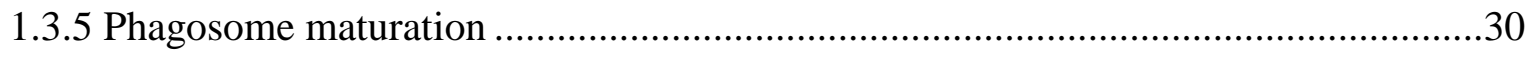

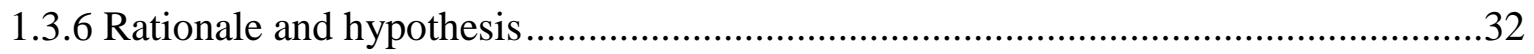

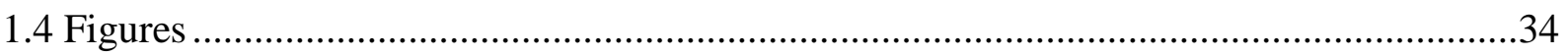

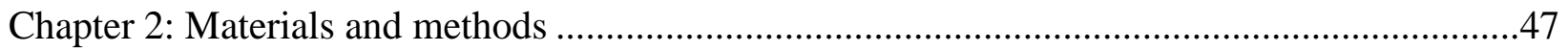

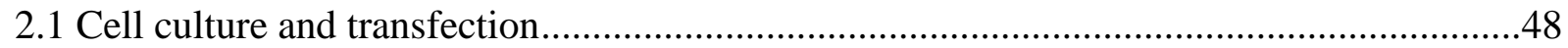


2.2 PIKfyve inhibition

2.3 Phagocytosis and endocytosis

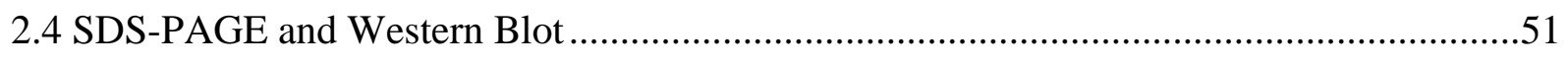

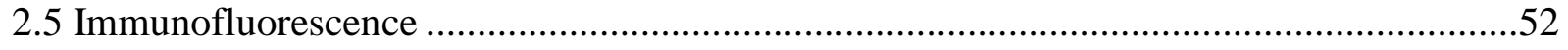

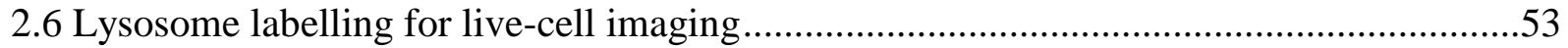

2.7 Epifluorescence and spinning disk confocal microscopy ...........................................53

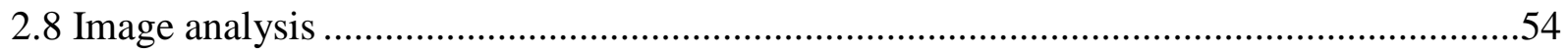

2.9 Quantitative Real Time Polymerase Chain Reaction .................................................55

2.10 Phosphoinositide labeling and measurement by HPLC-coupled flow scintillation ..........55

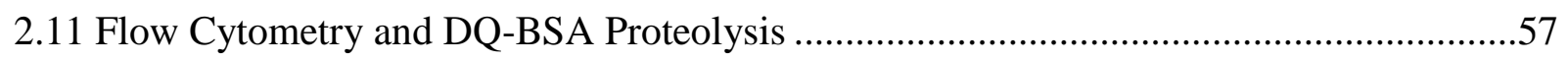

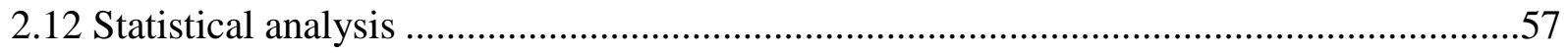

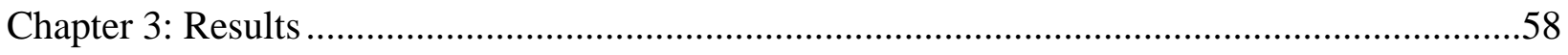

3.1 TFEB activation through PIKfyve inhibition does not contribute to lysosome enlargement

3.1.1 PIKfyve inhibition induces TFEB nuclear translocation ....................................59

3.1.2 PIKfyve inhibition increases dephosphorylation of TFEB .................................61

3.1.3 TFEB dephosphorylation may result from inhibition of GSK3 but not mTORC1 or

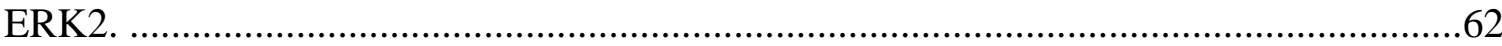

3.1.4 Acute PIKfyve inhibition does not increase lysosomal protein expression.............64

3.1.5 Biosynthesis is not required for acute lysosome enlargement in PIKfyve inhibited

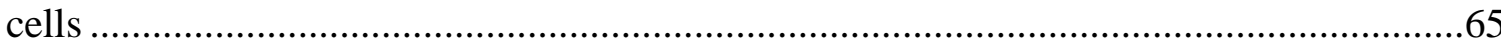

3.2 Phagocytosis induces TFEB nuclear localization and enhances proteolysis ...................69

3.2.1 Fc $\gamma$ receptor engagement leads to TFEB nuclear localization ..............................69

3.2.2 Lysosomes become more proteolytic after TFEB-induced lysosome biogenesis ......70

3.2.3 Phagocytosis leads to TFEB dephosphorylation .............................................. 71

3.2.4 Fc $\gamma$ receptor signaling leading to actin polymerization is required for TFEB nuclear

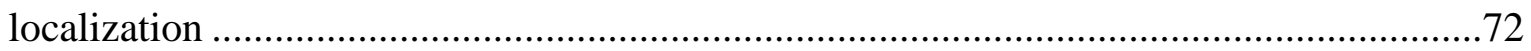

3.2.5 Particle internalisation but not phagosome maturation is required for TFEB

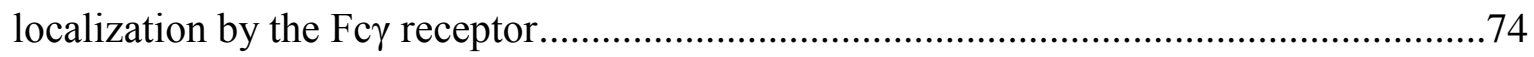

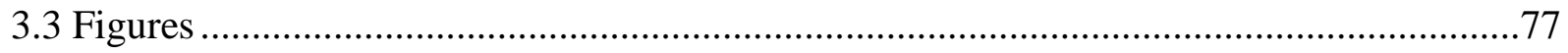

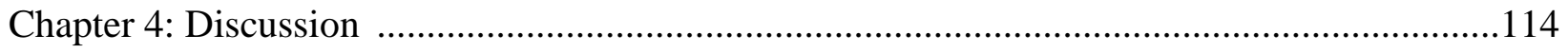

vii 


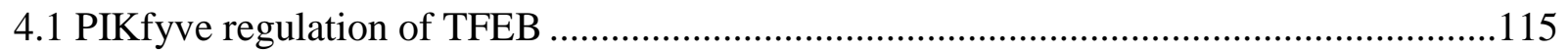

4.1.1 TFEB is not required for PIKfyve inhibition-induced lysosome enlargement ........115

4.1.2 PIKfyve regulation of mTORC 1 ...................................................................117

4.1.3 PIKfyve regulation of other known TFEB kinases ...........................................119

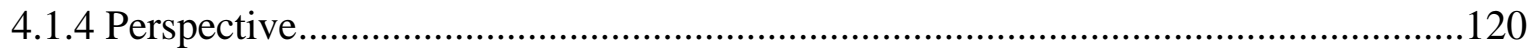

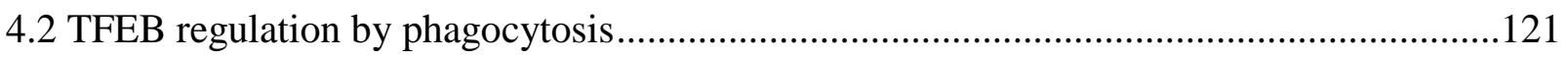

4.2.1 Phagocytosis activates TFEB to enhance proteolysis .......................................121

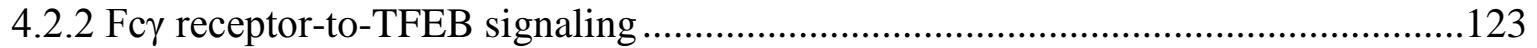

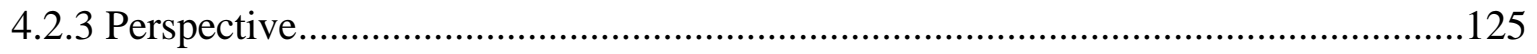

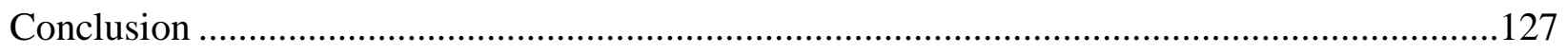

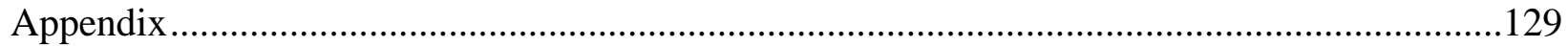

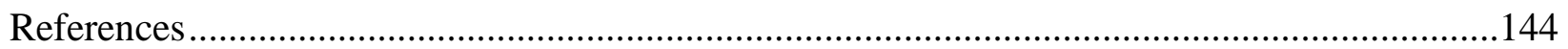




\section{List of Figures}

Figure 1.1.1: TFEB regulation by mTORC1 and nutrients .35

Figure 1.1.2: Regulation of TFEB by post-translational modifications.

Figure 1.2.1: Phosphatidylinositol structure and localization of its phosphorylated derivatives ..39

Figure 1.2.2: Synthesis and turnover of $\operatorname{PtdIns}(3,5) P_{2}$

Figure 1.3.1: Basic structure of typical immunoglobulin $\mathrm{G}$

Figure 1.3.2: Fc $\gamma$ receptor signaling .45

Figure 3.1.1: Apilimod depletes PtdIns(3,5) $P_{2}$ in RAW macrophages leading to enlarged lysosomes .78

Figure 3.1.2: PIKfyve inhibition with apilimod induces TFEB nuclear localization .80

Figure 3.1.3: PIKfyve inhibition also induces nuclear localization of other MiT/TFE family transcription factors .82

Figure 3.1.4: Apilimod treatment changes TFEB phosphorylation pattern ...............................84

Figure 3.1.5: PIKfyve and mTORC1 function independently .............................................86

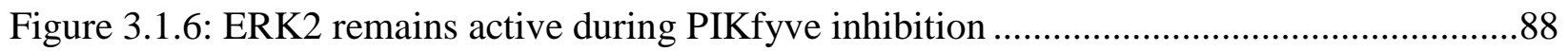

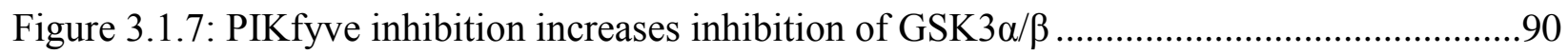

Figure 3.1.8: PIKfyve inhibition increases lysosome gene transcription but not translation ........92 Figure 3.1.9: Lysosome enlargement during PIKfyve inhibition is not abated by $t f e b^{-/}$and or $\mathrm{tfe}^{-\mathrm{-}-}$

Figure 3.1.10: Protein synthesis does not contribute to lysosome enlargement during PIKfyve inhibition

Figure 3.2.1: Engagement of the Fc $\gamma$ receptor induces TFEB nuclear localization .98

Figure 3.2.2: Cytokine treatment does not induce TFEB nuclear translocation 100

Figure 3.2.3: TFEB activation with Fc $\gamma$ receptor engagement enhances lysosome proteolysis ..102

Figure 3.2.4: TFEB phosphorylation changes following phagocytosis 104

Figure 3.2.5: mTORC1 activity does not decrease following phagocytosis 106

Figure 3.2.6: Fc $\gamma$ receptor signaling appears necessary for TFEB nuclear localization 108

Figure 3.2.7: Particle internalization is required for TFEB nuclear localization 110

Figure 3.2.8: Rab7 is required for TFEB nuclear localization with $E$. coli .112 


\section{List of Appendices}

Figure A1.1: Effect of constitutively active GSK3 $\beta$ on TFEB nuclear localization ..................130

Figure A1.2: Triple knockout of MiT/TFE family members does not reduce lysosome

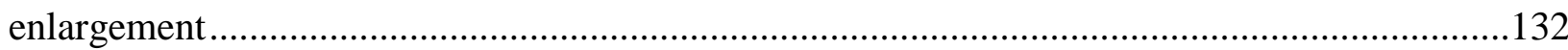

Figure A1.3: Fc $\gamma$ receptor engagement does not change lysosome number ............................134

Figure A1.4: Frustrated phagocytosis activates Fc $\gamma$ receptor signaling but does not activate TFEB

Figure A2.1: Fcy receptor phagocytosis increases protein abundance of some lysosomal proteins

Figure A2.2: Pre-stimulation of the Fc $\gamma$ receptor enhances bacterial killing ...........................140

Figure A2.3: TFEB nuclear localization depends on $\mathrm{Ca}^{2+}$ release from mucolipin-1 


\section{List of Abbreviations}

4E-BP1 - Eukaryotic translation initiation factor 4E-binding protein 1

AGS3 - activator of G-protein signaling 3

bHLH - basic helix loop helix

CASTOR - cytosolic arginine sensor for mTORC1 subunit 1

CLEAR - coordinated lysosomal enhancement and regulation

CPS - carboxypeptidase S

DAG - diacylglycerol

Deptor - DEP-domain-containing mTOR-interacting protein

EEA1 - early endosome antigen 1

ERK - extracellular signal-regulated kinase

ESCRT - endosomal sorting complexes required for transport

FYVE - (Fab1, YOTB, Vac1, EEA1)

GAP - GTPase-activating protein

$\mathrm{GEF}$ - guanine nucleotide exchange factor

GSK3 - glycogen synthase kinase 3

HLH - helix loop helix

HOPS - homotypic fusion and vacuole protein sorting

IgG - immunoglobulin G

IL - interleukin

$\mathrm{IP}_{3}$ - inositol trisphosphate

ITAM - immunoreceptor tyrosine-based activation motif

ITIM - immunoreceptor tyrosine-based inhibitory motif

LPS - lipopolysaccharide

LAT - linker of activated T cells

LZ - leucine zipper

M6P - mannose 6-phosphate

MiTF - melanogenesis associated transcription factor

mLST8 - mammalian lethal with Sec13 protein 8

mSin1 - mammalian stress-activated protein kinase interacting protein 
MTMR - myotubularin-related

mTOR - mechanistic target of rapamycin

mTORC1/2 - mTOR complex $1 / 2$

MVB - multivesicular body

NLS - nuclear localization sequence

$\mathrm{NPF}$ - nucleation promotion factor

PAMP - pathogen-associated membrane protein

$\mathrm{PH}$ - Pleckstrin homology

PLC/PLD - phospholipase C/D

$\mathrm{PKC} / \mathrm{PKD}$ - protein kinase C/D

PP2A - protein phosphatase $2 \mathrm{~A}$

PRAS40 - proline-rich Akt1 substrate of $40 \mathrm{kDa}$

PRR - pattern recognition receptors

PtdIns - phosphatidylinositol

PtdInsPs - phosphoinositides

PX - Phox homology

RANKL - receptor activator of nuclear factor kappa-B ligand

Raptor - regulatory associated protein of mTOR

Rictor - rapamycin insensitive component of TOR

S6K - ribosomal protein S6 kinase

SH2 - Src-homology 2

SNARE - soluble N-ethylmaleimide-sensitive factor attachment protein receptors

SREBP1 - sterol regulatory element-binding transcription factor 1

SUMO - small ubiquitin-like modifier

Syk - spleen tyrosine kinase

TPC1/2 - two pore channel $1 / 2$

TSC1/2 - tuberous sclerosis protein $1 / 2$

TFE3 - transcription factor E3

TFEB - transcription factor EB

TFEC - transcription factor EC

TRPML1 - transient receptor potential mucolipin-1 
TORC 1 - target of rapamycin

ULK1 - UNC-51-like kinase 1

V-ATPase - vacuolar ATPase

Vps - vacuolar protein sorting protein

WASp - Wiskott-Aldrich Syndrome protein 
Chapter 1: Introduction 


\subsection{The lysosome}

The lysosome is an acidic organelle and degradation center of the cell. The acidic lumen is generated and maintained by the vacuolar $\mathrm{H}^{+}$-ATPase (V-ATPase). Protons are pumped into the lysosome at the expensive of ATP to a $\mathrm{pH}$ of 4.5-5.0. This acidic environment serves as the optimal environment for over 60 hydrolytic enzymes, including proteases, lipases, and nucleases (Schröder, Wrocklage, Hasilik, \& Saftig, 2010). The concerted action of these hydrolases leads to the degradation of an array of complex macromolecules into their constituents. These macromolecules can be delivered to the lysosome by a variety of degradative pathways. Extracellular materials can be internalized through receptor-mediated endocytosis, phagocytosis or pinocytosis and are trafficked through the endocytic pathway (described in greater detail below) (Bohdanowicz \& Grinstein, 2013). Membrane components can also be trafficked through the endocytic pathway, sequestered and delivered as intraluminal vesicles (Henne, Buchkovich, \& Emr, 2011). Finally, soluble cytosolic macromolecules or organelles are delivered to the lysosome through autophagy (Saha, Panigrahi, Patil, \& Bhutia, 2018). The resulting degradation products, like amino acids, can then be reused by the cell and transported out of the lysosome through numerous transporters (Schröder et al., 2010). The lysosome is also known to contain many ions and solutes that facilitate other lysosomal functions, including endomembrane trafficking, lysosome exocytosis, and cellular signaling (Xiong \& Zhu, 2016).

\subsubsection{The lysosome as a signaling hub}

Beyond its degradative function, the lysosome participates in signaling by serving as a signaling platform or by activating signaling mediators from lysosomal ions. The most wellknown signaling hub found at the lysosome is the mechanistic target of rapamycin complex 1 
(mTORC1), which localizes directly to the lysosomal surface (Sancak et al., 2010). This complex is made up of the mTOR kinase at its core, associated with Raptor (regulatory associated protein of mTOR), PRAS40 (proline-rich Akt1 substrate of $40 \mathrm{kDa}$ ), mLST8 (mammalian lethal with Sec13 protein 8), and Deptor (DEP-domain-containing mTORinteracting protein). mTOR can also form a second non-lysosomal complex, mTORC2, when associated with Rictor (rapamycin insensitive component of TOR), mLST8, mSin1 (mammalian stress-activated protein kinase interacting protein) and Deptor (Saxton \& Sabatini, 2017). The activity of mTORC1 is dependent on two nutrient-based inputs that converge at the lysosome: amino acids and growth factors.

Amino acids influence the lysosomal localization of mTORC1 by acting on the Rag GTPases (Sancak et al., 2008). These GTPases act as heterodimers in opposing nucleotide binding states. When active, GTP-bound Rag A or B heterodimerize with GDP-bound Rag C or D (Sancak et al., 2008). Dedicated sensors for leucine and arginine sense the abundance of amino acids and influence the nucleotide binding state of Rag A/B. Specifically, cytosolic leucine and arginine are sensed by Sestrin2 and CASTOR respectively leading to inhibition of GATOR1, a GTPase-activating protein (GAP) for Rag A/B (Chantranupong et al., 2016; Saxton et al., 2016; Wolfson et al., 2016). Additionally, amino acids within the lysosome are also sensed. Arginine levels are sensed by the permease SLC38A9 and general amino acids are sensed by the VATPase (Rebsamen et al., 2015; S. Wang et al., 2015; Zoncu et al., 2011). Together, these two components activate the Ragulator complex, a guanine exchange factor (GEF) for Rag A/B (BarPeled, Schweitzer, Zoncu, \& Sabatini, 2012). The Ragulator complex also serves as a docking site for activated Rag GTPases, which brings mTORC1 to the lysosome through interactions with Raptor and Ragulator (Sancak et al., 2010). Once recruited to the lysosome, mTORC1 can 
then be activated by growth factors in a Rheb GTPase-dependent manner (Garami et al., 2003). Growth factor signaling leads to inhibition of the Rheb-specific GAP, tuberous sclerosis complex 1/2 (TSC1/2), leading to GTP-loading of Rheb and activation of mTORC1 (Garami et al., 2003).

In turn, activated mTORC1 promotes cell growth and proliferation by promoting processes like protein and lipid synthesis. Protein synthesis is stimulated through the phosphorylation of p70-S6 kinase (S6K) and eukaryotic translation initiation factor 4E-binding protein 1 (4E-BP1) (Brown et al., 1995; Brunn et al., 1997). Together, these factors upregulate ribosomes and other components of the translational machinery. Similarly, lipid synthesis is promoted through the activation and nuclear localization of the transcription factor sterol regulatory element-binding transcription factor 1 (SREBP1), a transcriptional regulator of lipid and sterol production (Porstmann et al., 2008). Simultaneously, mTORC1 also blocks catabolic processes by downregulating autophagy, in part by inhibiting substrates like ULK1 (Hosokawa et al., 2009).

In contrast to mTORC1, lysosomal ions also play a significant role in cellular signaling and membrane trafficking. The lumen of the lysosome is a significant storage site for many ions including $\mathrm{H}^{+}, \mathrm{Ca}^{2+}, \mathrm{Na}^{+}, \mathrm{Cl}^{-}$, and $\mathrm{Fe}^{+}$(Xiong \& Zhu, 2016). Specifically, the concentration of $\mathrm{Ca}^{2+}$ in the lysosome is around $500 \mu \mathrm{M}$, much greater than the cytosolic $\mathrm{Ca}^{2+}$ concentration (Christensen, Myers, \& Swanson, 2002). Release of $\mathrm{Ca}^{2+}$ from the lysosomal lumen can be facilitated by transient receptor potential mucolipin-1 (TRPML1) or P2X4 (Bo et al., 2003; Dong et al., 2010). The efflux of $\mathrm{Ca}^{2+}$ from the lysosome to the cytosol through TRPML1 can participate in cellular signaling by activating the protein phosphatase calcineurin (Medina et al., 2015). Common to both mTORC1 and lysosomal $\mathrm{Ca}^{2+}$-dependent calcineurin activation, lysosome biogenesis and autophagy can be controlled at a transcriptional level by regulating the 
transcription factor EB (TFEB), a member of the MiT/TFE family of transcription factors (Medina et al., 2015; Sardiello et al., 2009).

\subsubsection{The MiT/TFE family of transcription factors}

The MiT/TFE family of transcription factors are key biosynthetic regulators of lysosomes, autophagy, and other metabolic processes (Slade \& Pulinilkunnil, 2017;

Steingrímsson, Copeland, \& Jenkins, 2004). In mammalian cells, this family of transcription factors is composed of four members: MiTF, TFE3, TFEB, and TFEC (Hemesath et al., 1994). While MiTF and TFEC are expressed in specific tissues, TFEB and TFE3 are expressed more ubiquitously (Slade \& Pulinilkunnil, 2017). Structurally, these transcription factors contain a helix-loop-helix (HLH) domain and a leucine zipper that facilitate homo- and heterodimerization between the family members (Hemesath et al., 1994). The MiT/TFE family exclusively heterodimerize amongst themselves due to a three residue shift found within the leucine zipper, excluding other HLH transcription factors from dimerizing (Pogenberg et al., 2012). They also contain a basic domain that is necessary for DNA binding, specifically towards E-box motifs (5'CANNTG-3'), characteristic of HLH/leucine-zipper transcription factors (Aksan \& Goding, 1998; Hemesath et al., 1994; Massari \& Murre, 2000). Despite their similarity, only MiTF, TFEB and TFE3 contain an transactivation domain necessary for transcriptional activation (Beckmann, Su, \& Kadesch, 1990). Conversely, TFEC lacks this domain and restricts transcriptional activation when dimerized with other MiT/TFE family members (Zhao, Zhao, Zhou, Mattei, \& de Crombrugghe, 1993). These genes also exist in invertebrates, albeit their collective function appear to be carried out by a single homologous gene. Drosophila melanogaster have a single MiTF gene (Bouché et al., 2016) while Caenorhabditis elegans has 
HLH-30 (Visvikis et al., 2014). Of the previously mentioned mammalian MiT/TFE family members, TFEB was the first to be characterized as a regulator of lysosomal gene expression (Sardiello et al., 2009), although others have shown that MiTF and TFE3 can also enhance lysosomal biogenesis in a cooperative manner (Martina et al., 2014; Ploper et al., 2015). Thus, TFEB will be the focus for this thesis.

Analysis of several lysosomal genes identified a common element within their promotor sequence, which the Ballabio group named the coordinated lysosomal enhancement and regulation (CLEAR) motif (5'-GTCACGTGAC-3') (Sardiello et al., 2009). Genome-wide analysis revealed 471 genes with one or more CLEAR motifs that are collectively controlled by TFEB (Palmieri et al., 2011). While some genes are responsible for functions such as vesicular trafficking or immunity, the largest proportion of these genes relate directly to the lysosome and autophagy (Palmieri et al., 2011). Therefore, TFEB is widely regarded as a master regulator of lysosome biogenesis and autophagy. Research has since focused on understanding how TFEB can be regulated and the specific contexts that benefit from modulating lysosome biogenesis.

\section{$\underline{1.1 .3}$ TFEB in autophagy and mTORC1 regulation}

The role of TFEB is best understood under the context of amino acid starvation (Figure 1.1.1). When amino acids are present, TFEB is inactive and restricted from the nucleus. Conversely, when amino acids are withdrawn, TFEB enters the nucleus where it can activate transcription of CLEAR network genes (Roczniak-Ferguson et al., 2012). The details regarding amino acid starvation have been well documented with TFEB subjected to multiphasic regulation. 
The presence of amino acids promotes the phosphorylation of TFEB at S3 by MAP4K3, mediating the interaction between TFEB and the activated Rag GTPases, where Rag A/B is GTP loaded and Rag C/D is GDP loaded (Hsu et al., 2018; Martina \& Puertollano, 2013). The active Rags recruit TFEB and mTORC1 to the Ragulator complex at the lysosome, where mTORC1 is further activated by the GTPase Rheb (Martina \& Puertollano, 2013; Sancak et al., 2010). At the lysosome, mTORC1 directly phosphorylates TFEB on at least three residues: S122, S142, and S211 (Roczniak-Ferguson et al., 2012; Vega-Rubin-de-Celis, Peña-Llopis, Konda, \& Brugarolas, 2017). The most important phosphorylation site modulating nuclear localization appears to be S211. Phospho-S211 creates a binding site for the chaperone 14-3-3/YHWH, which masks the nuclear localization sequence (NLS) found at amino acids 241-252 of TFEB (RoczniakFerguson et al., 2012). As a result, TFEB remains in the cytosol and transcription of CLEAR network genes is restricted.

Conversely, amino acid starvation prevents the activation of Rag GTPases (Martina \& Puertollano, 2013). Here, Rag A/B are GDP-loaded and Rag C/D are GTP-loaded. mTORC1 is inactive and cannot phosphorylate TFEB. At the same time, amino acid starvation leads to the efflux of $\mathrm{Ca}^{2+}$ out of the lysosome, through the gated-calcium channel TRPML1 (Medina et al., 2015). Release of $\mathrm{Ca}^{2+}$ activates the protein phosphatase calcineurin, responsible for dephosphorylation of TFEB at S211 (Medina et al., 2015). Without phospho-S211, 14-3-3 cannot bind to TFEB and the NLS becomes exposed (Roczniak-Ferguson et al., 2012). Nuclear translocation of TFEB may be facilitated by importin-8 (Perera et al., 2015), where TFEB enhances transcription of CLEAR network genes. Therefore, increasing TFEB-mediated lysosomal biosynthesis promotes degradation of autophagic cargo to be recycled by the cell. 
Beyond nutritional stress, TFEB activation appears to have significant implications towards numerous cellular stressors, many of which rely on mTORC1 independent routes (Figure 1.1.2).

\subsubsection{Regulation of TFEB by phosphorylation}

There are at least six kinases and two phosphatases that can directly modulate TFEB phosphorylation thus far (Puertollano, Ferguson, Brugarolas, \& Ballabio, 2018). Like mTORC1, many phosphorylation events can control subcellular localization of TFEB. One such kinase is Akt, that phosphorylates TFEB at S467 and was investigated as a therapeutic target towards neurodegenerative diseases, including Huntington's disease, Parkinson's disease, and Alzheimer's disease (Palmieri et al., 2017; Sarkar, Davies, Huang, Tunnacliffe, \& Rubinsztein, 2007). These neurodegenerative diseases stem from the accumulation of aberrant protein aggregates in their cytosol that are typically cleared by autophagy. Thus, disruption to autophagy has been linked to disease on-set (Nixon, 2013). Many neurodegenerative diseases also appear to have impaired or insufficient TFEB activation. (Decressac et al., 2013; Polito et al., 2014; Xiao et al., 2014). To enhance autophagy in neurons, TFEB can be targeted by inhibiting Akt with trehalose, a non-reducing disaccharide (Palmieri et al., 2017; Sarkar et al., 2007). Akt inhibition with trehalose leads to TFEB dephosphorylation at S467 and nuclear localization, independently of mTORC1 (Palmieri et al., 2017).

Another kinase responsible for modulating TFEB localization is the glycogen synthase kinase $3 \beta$ (GSK3 $\beta$ ), which directly phosphorylates S134 and S138 (Y. Li et al., 2016; Marchand, Arsenault, Raymond-Fleury, Boisvert, \& Boucher, 2015). Inhibition of GSK3 $\beta$ leads to TFEB nuclear translocation, enhancing autophagy in cancer cells and neurodegenerative disorders (Y. Li et al., 2016; Marchand et al., 2015). Here, GSK3 $\beta$ may specifically regulate 
TFEB nuclear export (L. Li et al., 2018). Interestingly, TFEB has long been known to be associated with renal cell carcinoma due to chromosomal rearrangement, leading to TFEB overexpression (Argani et al., 2016; Kuiper et al., 2003). While TFEB activation may induce some cancers, TFEB activation is necessary to induce cell death in other cancers (Gayle et al., 2017). Nevertheless, the effect of GSK $3 \beta$ on TFEB can further be regulated upstream by protein kinase C- $\alpha(\mathrm{PKC} \alpha)$ or PKC $\delta$ (Y. Li et al., 2016). Here, enhanced PKC $\alpha$ or PKC $\delta$ activation can phosphorylate and inhibit GSK3 $\beta$, leading to TFEB activation.

Certain isoforms of PKC may also have important roles in TFEB-dependent immune function, including PKC $\alpha$ and/or PKC $\gamma$. Mouse macrophages challenged with Salmonella enterica or Staphylococcus aureus induced TFEB nuclear localization (Najibi, Labed, Visvikis, \& Irazoqui, 2016; Visvikis et al., 2014). This effect depended on the activity of multiple signaling regulators including phosphatidylcholine-specific phospholipase C (PLC), protein kinase D (PKD), and PKC $\alpha$ and/or PKC $\gamma$, independently of mTORC1 (Najibi et al., 2016). The authors speculate that this pathway may be downstream of G-protein coupled receptor activation (Najibi et al., 2016). Consistently, the activator of G-protein signaling 3 (AGS3) appears to increase expression upon stimulation with lipopolysaccharide (LPS) or live bacteria (Vural et al., 2016). However, contrary to the previous study, the effects of AGS3 appear to inhibit mTORC1 through inhibition of the Rheb via TSC1/2, similar to growth factor signaling (Vural et al., 2016). Nevertheless, immune signaling appears to be a potent activator of TFEB activation.

Other PKC isoforms can also regulate TFEB. Osteoclasts are bone absorbing cells that rely on lysosome exocytosis for reabsorption. Cytokine activation by receptor activator of nuclear factor kappa-B ligand (RANKL) in osteoclasts induces PKC $\beta$ activation and phosphorylation of TFEB at S462 and/or S463, S466/S467, and S469 (Ferron et al., 2013). 
Interestingly, TFEB phosphorylation by PKC $\beta$ stabilized the protein and total cellular accumulation of TFEB (Ferron et al., 2013).

Additionally, TFEB activation appears to play significant roles in the cell's response to other stressors. Induction of mitochondrial stress (Ivankovic, Chau, Schapira, \& Gegg, 2016; Nezich, Wang, Fogel, \& Youle, 2015), endoplasmic reticulum stress (Martina, Diab, Brady, \& Puertollano, 2016), or oxidative stress (Martina \& Puertollano, 2018) can all induce TFEB activation. During oxidative stress, S211 becomes dephosphorylated by protein phosphatase 2A (PP2A), inducing TFEB nuclear localization (Martina \& Puertollano, 2018). PP2A activity may also be activated during long term fasting in animal models, leading to TFEB dephosphorylation (Chen et al., 2017). S211 is not the only phospho-site with multiple regulators. The extracellular signal-regulated kinases 2 (ERK2) was suggested to phosphorylate S142, which prevented TFEB nuclear localization (Settembre et al., 2011). However, the exact role of ERK2 on S142 phosphorylation is not well understood as follow up studies with an ERK specific inhibitor did not induce TFEB activation (Settembre et al., 2012).

\subsubsection{Regulation of TFEB by other post-translational modifications}

While phosphorylation remains the dominant post-translational modification determining TFEB localization and stability, other post-translational modifications appear to alter activity of the transcription factor within the nucleus. Sumoylation was observed in MiTF, TFEB, and TFE3 (Miller, Levy, Davis, Razin, \& Fisher, 2005). The addition of small ubiquitin-like modifier (SUMO) to lysine residues altered their ability to bind specific promoters. Sumoylated MiTF at K182 and K316 displayed stronger binding to promoters with multiple E-box sequences, but was not required for heterodimerization or nuclear translocation (Miller et al., 2005). TFEB appears 
to retain a single sumo-site at K347, which was analogous to K316 of MiTF by sequence alignment (Miller et al., 2005). Additionally, acetylation of TFEB was also observed. Deacetylation at K116 by the nuclear deacetylase SIRT1 enhances TFEB-mediated transcription and promotes clearance of fibrillar amyloid beta plaques associated with Alzheimer's disease (Bao et al., 2016). Conversely, acetylation of TFEB at K91, K103, K116 and K430 was reported to enhance transcription of CLEAR network genes (J. Zhang et al., 2018). These residues may be modulated by acetyl-Coenzyme A acetyltransferase 1 and histone deacetylase (J. Zhang et al., 2018).

Together, TFEB activation appears to be a global adaptation response to cellular stress and a viable option to combat a variety of diseases. The current list of TFEB regulators and TFEB-dependent pathways is unlikely exhaustive. Here, we explore two possible pathways that may depend on TFEB activation. In section 1.2, we explore the role of TFEB in lysosome size dysregulation due to loss of a lysosomal lipid. In section 1.3, we examine the contribution of TFEB on immunoglobulin G-mediated phagocytosis. 


\subsection{Regulation of lysosome morphology by phosphatidylinositol 3,5-bisphosphate}

Lysosomes are highly heterogenous organelles. Their subcellular position can change, altering their $\mathrm{pH}$ and degradative capacity (Johnson, Ostrowski, Jaumouillé, \& Grinstein, 2016). While lysosome number may reach into the hundreds in mammalian cells, other species like Saccharomyces cerevisiae often have a single, multilobed vacuole (D Sbrissa, Ikonomov, \& Shisheva, 1999). Neutrophils have multiple lysosome-related organelles, each with specialized function (Cowland \& Borregaard, 2016). Lysosomes are also highly adaptive and can respond to their environment. Immune stimuli are sensed by dendritic cells, inducing reorganisation of spherical lysosomes into a vast tubular network (Mrakovic, Kay, Furuya, Brumell, \& Botelho, 2012). Evidently, lysosomes require exquisite regulation to ensure form and function. The number and size of lysosomes remain relatively constant in any given cell type under basal conditions. This is despite extracellular materials and newly synthesized proteins constantly being delivered towards the lysosome by vesicular traffic, driven by the endocytic pathway and biosynthetic pathway from the trans-Golgi network.

\subsubsection{Endocytic pathway}

Solutes may be captured from the extracellular milieu including cofactors like iron or growth factors like epidermal growth factor. Capture of these solutes can be facilitated by specific receptors by receptor-mediated endocytosis or non-specifically through pinocytosis. Additionally, invagination of the plasma membrane can be mediated by coat proteins like clathrin (Abe, Inoue, Galvez, Klein, \& Meyer, 2008). These plasma membrane-derived invaginations pinch and form endocytic vesicles in the cytosol where they fuse with early endosomes. Here, the cargo and their respective receptors are sorted towards divergent paths. 
Membrane bound proteins, like receptors, can be recycled and sorted back to the plasma membrane. In contrast, membrane proteins destined for degradation are ubiquitinated and sorted into intraluminal vesicles by the endosomal sorting complexes required for transport (ESCRT) complex (Katzmann, Babst, \& Emr, 2001). The early endosomes can also fuse with each other by interacting with early endosome antigen 1 (EEA1) (Christoforidis, McBride, Burgoyne, \& Zerial, 1999). Fission of early endosomes sends cargo to their respective destinations throughout the cell.

As the endosome matures towards the lysosome, early endosomal markers are exchanged for late endosomal markers, including exchange of Rab GTPases from Rab5 to Rab7 respectively (described in greater detail below in the context of phagocytosis) (Rink, Ghigo, Kalaidzidis, \& Zerial, 2005). The homotypic fusion and vacuole protein sorting (HOPS) complex facilitates homotypic fusion between Rab7 positive vesicles including late endosomes and lysosomes (Seals, Eitzen, Margolis, Wickner, \& Price, 2000). The resulting endolysosome facilitates the degradation of internalized solutes and formed intraluminal vesicles by the various hydrolases. While fusion with the lysosome is often discussed as the final trafficking step in the pathway, mounting evidence supports the recycling of "pure" lysosomes from hybrid endolysosomal degradative compartments (Bissig, Hurbain, Raposo, \& van Niel, 2017; Bright, Davis, \& Luzio, 2016; Krishna et al., 2016). Compared to the degradative endolysosomes, "pure" lysosomes may be hydrolytically inactive and may serve as terminal storage lysosomes that recapture and concentrate hydrolases for further rounds of fusion and degradation (Bissig et al., 2017; Bright et al., 2016). How endolysosomes are resolved remains relatively unknown. 


\subsubsection{Biosynthetic pathway and recycling pathways}

Newly synthesized materials are made by the ribosomes in the endoplasmic reticulum and further processed in the Golgi. Many luminal proteins are modified with glycan chains that feature a mannose 6-phosphate which is targeted for delivery by the mannose 6-phosphate receptor (Braulke \& Bonifacino, 2009). These cargos exit the trans-Golgi network and fuse with the early endosome. The mild acidity of the early endosome induces disassociation of the cargo from the receptor. Similar to the endocytic pathway, the cargo and receptor can have divergent paths; the cargo can progress through the endocytic pathway towards the lysosome while the receptor can be recycled back to the trans-Golgi network for further rounds of trafficking. Recycling is mediated by the retromer complex, a pentameric complex composed of two subcomplexes. The vacuolar protein sorting (Vps) trimer of Vps35, Vps29 and Vps26 mediate cargo selection, while the SNX-BAR dimer of SNX1/2 and SNX 5/6 facilitate membrane bending (Carlton et al., 2004; Seaman, McCaffery, \& Emr, 1998; Wassmer et al., 2006).

Alternatively, membrane proteins are sorted to the lysosome based on the presence of sorting signals in their cytosolic tails. These sorting signals interact with clathrin adaptor proteins, recruiting the coat protein and inducing vesicle formation (Dietrich, Kastrup, Nielsen, Ødum, \& Geisler, 1997; Ohno et al., 1995). These vesicles can either be transported to the plasma membrane and trafficked through the endocytic pathway to reach the lysosome, or be transported directly to endosomes or the lysosome (Mathews, Martinie, \& Fambrough, 1992). Despite the numerous trafficking pathways throughout the cell, organelles appear to maintain their identity and accurately target delivery of cargo. A key determinant in maintaining membrane identity are the phosphoinositides. 


\subsubsection{Phosphoinositides}

Phosphoinositides (PtdInsPs) are low abundance membrane lipids involved in numerous cellular functions, including signal transduction, membrane trafficking, and cell motility (Balla, 2013). Existing on the cytosolic leaflet of cellular membranes, phosphatidylinositol (PtdIns) and its phosphorylated derivatives (PtdInsP) constitute 10-20\% of all phospholipids. Structurally, PtdInsPs feature a glycerol backbone attached to two fatty acid tails and a myo-inositol headgroup (Figure 1.2.1A). The most common fatty acid tail combination is 1-stearyol-2aracadonl, 38 carbons long with 4 double bonds combined, accounting for $35-75 \%$ of PtdIns/Ps in primary mammalian cells (Hicks, DeLong, Thomas, Samuel, \& Cui, 2006; Milne, Ivanova, DeCamp, Hsueh, \& Brown, 2005). Other fatty acid tails with varying chain lengths and/or degrees of unsaturation can also exist, conferring molecular heterogeneity to PtdIns/P that may influence effector binding (Bone et al., 2017; Schmid, Wise, Mitchell, Nussbaum, \& Woscholski, 2004; Shulga, Anderson, Topham, \& Epand, 2012).

Meanwhile, most research focuses on functional differences in inositol headgroup phosphorylation. In mammalian cells, reversible phosphorylation at the 3 , 4, or 5 position can yield three mono- $[\operatorname{PtdIns}(3) P, \operatorname{PtdIns}(4) P, \operatorname{PtdIns}(5) P]$, three bis- $\left[\operatorname{PtdIns}(3,4) P_{2}, \operatorname{PtdIns}(3,5) P_{2}\right.$, PtdIns $\left.(4,5) P_{2}\right]$ and one tris-phosphorylated species $\left[\operatorname{PtdIns}(3,4,5) P_{3}\right]$. By comparison, only PtdIns(3) $P$, PtdIns(4) $P$, PtdIns(3,5) $P_{2}$ and $\operatorname{Ptd} \operatorname{Ins}(4,5) P_{2}$ are detected in the budding yeast Saccharomyces cerevisiae. The conversion between PtdInsP species is mediated by 19 kinases and 28 phosphatases. These enzymes are grouped based on substrate specificity and structural domains, encompassing 8 classes of kinases and 10 classes of phosphatases in mammals (Sasaki et al., 2009). 
Each PtdInsP localizes to multiple organelles and recruits specific effectors (Figure 1.2.1B). Some PtdInsPs are concentrated at specific organelles to facilitate basal function. For example, PtdIns(4,5) $P_{2}$ regulates clathrin-mediated endocytosis at the plasma membrane (Abe et al., 2008), PtdIns(4) $P$ is found at the trans-Golgi network regulating trans-Golgi network-toendosome traffic (Y. J. Wang et al., 2003), and PtdIns(3)P controls endosome fusion at the early endosome (Lawe et al., 2002). However, many PtdInsPs are transiently generated in response to various stimuli and appear on alternative organelles. For instance, $\operatorname{PtdIns}(4) P$ may also be found at late endosomes and autophagosomes, coordinating endosomal trafficking to the lysosome and autophagosome-lysosome fusion (Craige, Salazar, \& Faundez, 2008; Jovic et al., 2012; Minogue, 2006; H. Wang et al., 2015).

These PtdInsPs act as membrane markers, recruiting specific subsets of effectors which often contain PtdInsP binding domains (Hammond \& Balla, 2015). To elicit different effects, PtdInsPs found at different membranes rely on coincidence detection. For example, PtdIns(3)P can recruit multiple effectors including EEA1 or Vps5 and Vps17. EEA1 shows specificity for early endosomes, binding to PtdIns(3)P via the FYVE (Fab1, YOTB, Vac1, EEA1) domain (Mu et al., 1995; Stenmark, Aasland, Toh, \& D’Arrigo, 1996). Interaction with PtdIns(3)P is insufficient for EEA1 function; the small GTPase Rab5 is also required for EEA1-mediated fusion of early endosomes (Lawe et al., 2002). In contrast, retrograde trafficking in yeast requires PtdIns(3)P dependent recruitment of retromer subunits Vps5 and Vps17 (Horazdovsky et al., 1997; Seaman et al., 1998). These proteins have a Phox homology (PX) domain that has low affinity for PtdIns(3)P individually (Horazdovsky et al., 1997). However, dimerized Vps5 and Vps17 have greater avidity for PtdIns(3)P and are recruited to the retromer trimer subcomplex to facilitate retrograde traffic from the endosomes (Seaman et al., 1998; Yu \& Lemmon, 2001). 
Thus, PtdInsPs can confer organelles with an identity and is intimately involved in regulating organelle function.

\subsubsection{Phosphatidylinositol 3,5-bisphosphate}

Lysosomes and late endosomes, in particular, are enriched with PtdIns $(3,5) P_{2}$. The primary route of PtdIns(3,5)P $P_{2}$ synthesis relies on the lipid kinase PIKfyve (Fab1 in yeast) (Figure 1.2.2) (Gary, Wurmser, Bonangelino, Weisman, \& Emr, 1998; Sbrissa, Ikonomov, \&

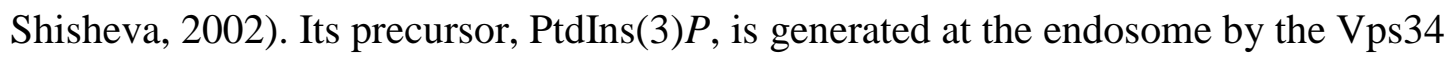
(Ikonomov et al., 2015; Schu et al., 1993). In turn, PtdIns(3)P is recognized by PIKfyve via its FYVE domain, and phosphorylates the inositol ring at the 5-position to yield $\operatorname{PtdIns}(3,5) P_{2}$ (Burd \& Emr, 1998; Ikonomov et al., 2015). Interestingly, PIKfyve forms a complex with its antagonizing phosphatase Sac3 (Fig4 in yeast), scaffolded by multiple copies of ArPIKfyve (Vac14 in yeast) (Alghamdi et al., 2013; Botelho, Efe, Teis, \& Emr, 2008; Gary et al., 2002; Natsuko Jin et al., 2008; Rudge, 2003; Sbrissa, Ikonomov, Fenner, \& Shisheva, 2008). Importantly, formation of the complete complex is necessary for PIKfyve activity; disruption to ArPIKfyve or Sac3 reduce PtdIns $(3,5) P_{2}$ levels, suggesting ArPIKfyve and Sac3 play positive regulatory functions on PIKfyve. In yeast, the Fab1 complex also includes Vac7 and Atg18, which positively and negatively regulate Fab1 function respectively (Dove et al., 2004; Natsuko Jin et al., 2008; Poirier, Eroglu, \& Marko, 2002). Alternatively, PtdIns(3,5) $P_{2}$ can be dephosphorylated by myotubularin-related (MTMR) 3-phosphatases to form PtdIns(5)P (Shen et al., 2009; D. M. Walker et al., 2001; Zolov et al., 2012). Regulation of PtdIns(3,5) $P_{2}$ synthesis is highly regulated and is required for proper cellular function. 


\subsubsection{Defects associated with PtdIns $(3,5) P_{2}$}

At an organism level, PtdIns $(3,5) P_{2}$ synthesis appears to be required for development. PIKfyve knockout lead to embryonic lethality (Ikonomov et al., 2011). Defective PtdIns(3,5) $P_{2}$ synthesis, induced by disrupting other components of the PIKfyve complex in mouse models, lead to reduced lifespan, neurological degeneration, insufficient myelination of axons and reduced coat colour (C. J. Ferguson et al., 2012; Lenk et al., 2016; Liggins et al., 2018). At a cellular level, defective PtdIns $(3,5) P_{2}$ synthesis abrogates certain trafficking pathways, lysosomal ion regulation, transcriptional regulation, and signaling at the lysosome.

One trafficking pathway dysregulated by the loss of $\operatorname{Ptd} \operatorname{Ins}(3,5) P_{2}$ is autophagy, the lysosome-dependent degradation of organelles or protein complexes. These materials are packaged into double membrane organelles known as autophagosomes that fuse with the lysosome. LC3-II is recruited to the autophagosomal membrane and is digested by the lysosome with the cargo of the autophagosome (Kabeya, 2000). Depletion of $\operatorname{PtdIns}(3,5) P_{2}$ by pharmacological inhibition of PIKfyve or in mutant primary mouse neuronal cells lead to an accumulation of autophagic markers including LC3-II (de Lartigue et al., 2009; Cole J. Ferguson, Lenk, \& Meisler, 2009; S. M. Kim et al., 2016). Furthermore, impairment of cargo degradation lead to a significant increase in LC3-II levels relative to PIKfyve inhibition alone (de Lartigue et al., 2009). Together, these results implicate PIKfyve in autophagosome degradation.

Lysosomal ion homeostasis is also regulated by $\operatorname{Ptd} \operatorname{Ins}(3,5) P_{2}$. Specifically, the $\mathrm{Ca}^{2+}$, $\mathrm{Na}^{+}$, and $\mathrm{K}^{+}$channels appear to be regulated by $\operatorname{PtdIns}(3,5) P_{2}$. The $\mathrm{Ca}^{2+}$ channel transient receptor potential ion channels mucolipin-1 (TRPML1) was shown to directly bind to PtdIns(3,5) $P_{2}$ via amino acids 1-69 of the N-terminal tail (Dong et al., 2010). Binding of TRPML1 by PtdIns $(3,5) P_{2}$ induced $\mathrm{Ca}^{2+}$ flux into the cytosol. Similarly, the $\mathrm{Na}^{+}$-selective two 
pore channel 1 and 2 (TPC1 \& TPC2) can also be activated by PtdIns(3,5) $P_{2}$ (X. Wang et al., 2012). In yeast, the $\mathrm{K}^{+} / \mathrm{H}^{+}$antiporter $\operatorname{Vnx} 1$ is also positively regulated by $\operatorname{Ptd} \operatorname{Ins}(3,5) P_{2}$ (Wilson, Scott, Dowell, \& Odorizzi, 2018). Assembly and activity of the V-ATPase was also suggested to be promoted by the presence of PtdIns $(3,5) P_{2}$, suggesting a role in acidification of the lysosome (S. C. Li et al., 2014). While others have suggested that lysosomes become basified (Dove et al., 2002; Jefferies et al., 2008), several groups showed that the yeast vacuole and mammalian lysosomes were not impaired when PtdIns(3,5) $P_{2}$ is lost (Ho, Choy, Wattson, Johnson, \& Botelho, 2015; Krishna et al., 2016; Wilson et al., 2018).

PtdIns(3,5) $P_{2}$ is also involved in transcriptional regulation in yeast. Tup1 and Cyc8 play dual roles in transcriptional repression and activation of over 150 genes, including the GAL1 gene in galactose metabolism (Han \& Emr, 2011). Here, the presence of glucose restricts galactose metabolism. Tup1-Cyc8 bind to promoters and recruit transcriptional repressors such as the DNA binding protein Mig1. When carbon sources shift, GAL1 gene is activated to promote galactose metabolism. PtdIns(3,5) $P_{2}$ at the vacuole recruits the repressive Tup1-Cyc8 complex and Cti6, facilitating their interaction. When associated with Cti6, Tup-Cyc8-Cti6 is a transcriptional activator and recruits other transcriptional activators like SAGA to promote activation of the same genes it previously suppressed (Han \& Emr, 2011). This conversion has been termed PtdIns(3,5)P $P_{2}$ dependent Tup1 conversion (PIPTC).

mTORC1 recruitment and activation may also be influenced by PtdIns(3,5) $P_{2}$ (Bridges et al., 2012; N. Jin et al., 2014). Adipocytes generate PtdIns(3,5) $P_{2}$ at the plasma membrane in response to insulin signaling, in a PI3-kinase class II $\alpha$ (PI3KC2 $\alpha$ ) dependent manner (Bridges et al., 2012). Recruitment of mTORC1 is mediated by direct interaction between $\operatorname{Ptd} \operatorname{Ins}(3,5) P_{2}$ and the WD40 domain of Raptor. This phenomenon is also observed in yeast, where TORC1 is 
recruited to the vacuole instead of the plasma membrane (N. Jin et al., 2014). Interestingly, PtdIns(3,5) $P_{2}$ also recruited substrates of TORC1, including the S6K yeast homolog Sch9.

\section{$\underline{1.2 .6 \text { PtdIns }(3,5) P_{2}} \underline{\text { modulates lysosome size }}$}

The most obvious phenotype from changes to PtdIns $(3,5) P_{2}$ levels is the modulation of lysosome size. Changes in lysosome size appear to be inversely correlated to the amount of PtdIns(3,5) $P_{2}$ in the cell. Perturbations to the PIKfyve/Fab1 complex lead to reduced PtdIns(3,5) $P_{2}$ levels and have swollen lysosomes (Rutherford, 2006; Yamamoto et al., 1995). The striking enlargement of lysosome size has been linked to several defects including lysosomal osmotic balance, retrograde traffic, intraluminal vesicle formation, and lysosome reformation.

Several lines of evidence suggest osmotic regulation is necessary for maintaining lysosome size. Strikingly, hyperosmotic shock increased PtdIns $(3,5) P_{2}$ levels 20 -fold in yeast, inducing vacuole fragmentation (Dove et al., 1997). The fragmentation of the vacuole was suggested to maintain cytosolic osmolarity by expelling water from the vacuole (Efe, Botelho, \& Emr, 2005). Furthermore, lysosome enlargement from PIKfyve inhibition can be abated by changes to various ion channels. Overexpression of TRPML1 or knockout of the subunits for a $\mathrm{Cl}^{-} / \mathrm{H}^{+}$exchanger, CLCN7 or OSTM1, abated lysosome enlargement during in fig $^{-/-}$or PIKfyve inhibited cells respectively (Dong et al., 2010; Gayle et al., 2017). Similarly, loss of function of the yeast $\mathrm{K}^{+} / \mathrm{H}^{+}$antiporter Vxn1 or V-ATPase subunit Vph1 also blocked lysosome enlargement during fablA (Wilson et al., 2018). Some of these channels may be necessary for $\mathrm{pH}$ regulation of the lysosome. Indeed, inhibition of the V-ATPase during PIKfyve inhibition can also block lysosome enlargement (Saveanu \& Lotersztajn, 2016; Schulze et al., 2017; Wilson et al., 2018). 
Proper membrane traffic is also necessary to maintain lysosome size. While endosomal trafficking to the lysosome and biosynthetic traffic from the Golgi to the lysosome appears normal, impaired retrograde traffic and MVB formation may contribute excess membrane that leads to lysosomal enlargement. (Bryant, Piper, Weisman, \& Stevens, 1998; Dove et al., 2002, 2004; Odorizzi, Babst, \& Emr, 1998; Rutherford, 2006). Retrograde traffic allows for recycling of receptors that deliver lysosome luminal components. In yeast, delivery of the hydrolase alkaline phosphatase bypasses the endosome-like prevacuolar compartment (PVC) to reach the vacuole directly and is retained on the vacuolar membrane. By fusing a retention sequence for recycling back to the trans-Golgi network, retention sequence alkaline phosphatase continues to traffic directly to the vacuole but is continuously retrieved from the vacuole and appears in the Golgi. Interestingly, mutation to vac7 induced accumulation of retention sequence ALP on the vacuolar membrane suggesting defects in recycling (Bryant et al., 1998). In mammalian cells, localization of cation independent mannose 6-phosphate receptor accumulated at the early endosome in PIKfyve knockdown cells, suggesting a failure in recycling to the trans-Golgi network (Rutherford, 2006).

A candidate protein regulating recycling is the Rab9 effector $\mathrm{p} 40$, which is necessary for retrograde traffic of the cation independent mannose 6-phosphate receptor (Ikonomov et al., 2003). Here, p40 was suggested to be phosphorylated by PIKfyve and interacts with Rab9 to promote retrograde traffic. Another key regulator of lysosome fission in yeast is Atg18. $\operatorname{atg} 184$ yeast have enlarged lysosomes while paradoxically having 5-10 times more $\operatorname{Ptd} \operatorname{Ins}(3,5) P_{2}$ compared to their wild type counterparts which persists even during hyperosmotic shock (Dove et al., 2004). Atg18 was suggested to be involved in membrane scission by the presence of an unstructured hydrophobic loop that folds into an $\alpha$-helix upon lipid binding (Gopaldass, Fauvet, 
Lashuel, Roux, \& Mayer, 2017). This $\alpha$-helix can insert into the membrane to promote membrane deformation. PtdIns $(3,5) P_{2}$ is necessary for oligomerization of Atg18, leading to tubulation of the membrane and subsequent membrane fission (Gopaldass et al., 2017; Scacioc et al., 2017). In mammalian cells, Atg18 has 4 homologs, the WD40 repeat protein interacting with phosphoinositides (WIPI) 1 through 4 (WIPI1-WIPI4) (Proikas-Cezanne, Takacs, Donnes, \& Kohlbacher, 2015). However, they have not been implicated in regulation of lysosome size (Krishna et al., 2016). Without the removal of membrane intended for retrograde traffic, the lysosomes appear to inherit the extra membrane contributing to lysosome enlargement.

MVB formation may also be impaired, leading to lysosome enlargement. Some hydrolases are expressed as transmembrane proteins and sorted into intraluminal vesicles by the ESCRT complex. Degradation of the vesicle liberates the hydrolase, allowing it to fill the vacuole. Using the polyphosphate phosphatase PHM5 as a model, Dove et al showed that PHM5 in $v a c 7 \Delta$ and $v a c 14 \Delta$ yeast was restricted to the limiting membrane of the vacuole, suggesting MVB formation was impaired (Dove et al., 2002). Similar defects can be seen tracking carboxypeptidase S (CPS) (Odorizzi et al., 1998). Without the ability to form intraluminal vesicles, membrane intended for degradation could contribute to lysosome enlargement.

Another possible reason for lysosome enlargement may be the inability for lysosomes to reform. Upon fusion with phagosomes and autophagosomes, lysosomes are reformed by various mechanisms, including budding and vesicle formation. Recent evidence suggests lysosomes are unable to reform when PIKfyve is inhibited (Bissig et al., 2017; Krishna et al., 2016). This failure to fission prevents endolysosomes from shrinking and may be a contributing factor to the swelling of lysosomes. 


\subsubsection{Rationale and hypothesis}

While the current understanding focuses on defects in membrane trafficking, the emergence of TFEB as a master regulator of lysosome biogenesis has yet to be considered. Two lines of evidence support a potential role for TFEB. First, TFEB activation by overexpression alone can increase lysosome number. Additionally, $\operatorname{Ptd} \operatorname{Ins}(3,5) P_{2}$ may indirectly modulate TFEB function through modulating mTORC1 activity (Bridges et al., 2012; N. Jin et al., 2014). In basal cells, PtdIns $(3,5) P_{2}$ is present and can activate mTORC1. In turn, mTORC1 suppresses TFEB function and lysosome biogenesis is off. Conversely, if PIKfyve is inhibited and PtdIns $(3,5) P_{2}$ levels decrease, mTORC1 can become less active promoting TFEB activity. Therefore, we hypothesize that PtdIns(3,5) $P_{2}$ can modulate TFEB nuclear localization may altering mTORC1 function. The resulting vacuolation phenotype may be enhanced by TFEB-mediated lysosome biogenesis.

To test this, we focus on three main objectives:

1) To determine whether TFEB and family members become active during PIKfyve inhibition;

2) To determine the signaling requirements for TFEB activation,

3) To quantify the relative contribution of TFEB on PIKfyve-induced lysosome enlargement. 


\subsection{The regulation of lysosome biogenesis in phagocytosis}

Phagocytosis is the actin-dependent engulfment of particles greater than $0.5 \mu \mathrm{m}$ in size. Unicellular eukaryotes, like the amoeba Dictyostelium, rely on phagocytosis to engulf bacteria as a food source (Cosson \& Soldati, 2008). In contrast, higher eukaryotes can employ phagocytosis for tissue maintenance. Various cell types, including epithelial cells, can phagocytose neighbouring apoptotic cells (Monks et al., 2005). Phagocytosis is also necessary for the immune system. Professional phagocytes, like macrophages, survey their environment and engulf any potential pathogens they encounter (Rabinovitch, 1995).

The recognition of various particles is mediated by interaction of specific receptors with ligands displayed on the surface of the particle. Phagocytes express many pattern recognition receptors (PRR) that collectively target conserved components of the pathogen, known as pathogen-associated molecular patterns (PAMP). For example, Gram negative bacteria can be targeted by the presence of cell wall component lipopolysaccharide (LPS). Recognition of LPS by scavenger receptor class A mediates phagocytosis of the bacteria (Peiser et al., 2002). Alternatively, phagocytes can recognize their prey through indirect means. Host-derived opsonins, including immunoglobulins and complement proteins, can bind to the pathogen and are recognized by a common set of receptors. Of the two, immunoglobulins target specific ligands on the pathogen. Immunoglobulins can be subdivided into five classes, with immunoglobulin $\mathrm{G}$ (IgG) representing the most abundant form (Vidarsson, Dekkers, \& Rispens, 2014).

\section{$\underline{1.3 .1 \text { Immunoglobulin } \mathrm{G}}$}

The best characterized opsonin-receptor pair is immunoglobulin $\mathrm{G}(\mathrm{IgG})$ and its corresponding Fc $\gamma$ receptor. IgG is a tetrameric protein consisting of two identical light chains 
and two identical heavy chains (Figure 1.3.1) (Gore, Andersson, Biran, Underwood, \& Riesenfeld, 2014). Each light chain consists of one variable region (VL) and one constant region (CL), while each heavy chain is composed of one variable region (VH) and three constant regions ( $\mathrm{CH} 1, \mathrm{CH} 2$, and $\mathrm{CH} 3)$. The resulting IgG molecule adopts a Y shape, with two fragment antigen binding $(\mathrm{Fab})$ regions and one fragment constant $(\mathrm{Fc})$ region. Each Fab region contains the variable region of one heavy chain and one light chain and the following constant region (VL-CL and VH-CH1). Together, these variable regions are responsible for binding of unique antigens. IgG can be subdivided into four subclasses in humans (IgG1, IgG2, IgG3, IgG4) and in mice (IgG1, IgG2a IgG2b IgG3). Major differences in the amino terminal $\mathrm{CH} 2$ domain and linker region between $\mathrm{CH} 1$ and $\mathrm{CH} 2$ can alter immune functions, such as complement or Fc $\gamma$ receptor binding (Canfield, 1991; Garred, Michaelsen, \& Aase, 1989). The Fc region, defined by the two $\mathrm{CH} 2-\mathrm{CH} 3$ regions of the heavy chain, is responsible for Fc $\gamma$ receptor binding. In addition, the presence of an N-linked glycan at Asn297 of the $\mathrm{CH} 2$ domain is required for Fc $\gamma$ receptor binding (M. R. Walker, Lund, Thompson, \& Jefferis, 1989).

\section{$\underline{1.3 .2 \mathrm{Fc} \gamma \text { receptors }}$}

The Fc $\gamma$ receptors are similarly diverse. Humans have three classes of Fc $\gamma \mathrm{R}$ subdivided into Fc $\gamma$ RI, Fc $\gamma$ RIIA, Fc $\gamma$ RIIB, Fc $\gamma$ RIIC, Fc $\gamma$ RIIIA, and Fc $\gamma$ RIIIB. Meanwhile, mice have four classes of Fc $\gamma$ R, composed of Fc $\gamma$ RI, Fc $\gamma$ RIIB, Fc $\gamma$ RIII, Fc $\gamma$ RIV. These Fc $\gamma$ R can be further classified based on whether they activate or inhibit signaling from the receptor. Receptors that activate signaling contain an immunoreceptor tyrosine-based activation motif (ITAM), characterized by tandem YxxI/L motifs. Meanwhile, inhibitory signals are mediated by the presence of an immunoreceptor tyrosine-based inhibition motif (ITIM). When the ITIM is 
activated, inositol and protein phosphatases are recruited and antagonize signaling from the ITAM (discussed further below) (D Cox, Dale, Kashiwada, Helgason, \& Greenberg, 2001; Joshi, Singh, Zulcic, \& Durden, 2014). The only receptor containing the inhibitory ITIM is FcyRIIB in both human and mouse. Therefore, relative expression of these receptors will dictate the types of responses from immune cells.

Of the receptors that activate signaling, only Fc $\gamma R I$ has high affinity for IgG while all others have lower affinity. Thus, engagement of the particle with a single $\mathrm{Fc} \gamma \mathrm{R}$ is unable to maintain ligand-receptor binding. Phagocytes actively probe their environment with actin-rich membrane ruffles and filopodia to aide engagement of multiple receptors with an opsonized particle (Flannagan, Harrison, Yip, Jaqaman, \& Grinstein, 2010). Engagement of a multivalent prey allows receptor clustering and initiation of downstream signaling starting with phosphorylation of ITAM domains (Sobota et al., 2005) (Figure 1.3.2). Phosphorylation of the tandem ITAMs is mediated by Src family kinases (Ghazizadeh, Bolen, \& Fleit, 1994; Hamada, Aoki, Akiyama, \& Toyoshima, 1993; Ibarrola et al., 1997; Suzuki et al., 2000). In turn, phosphoITAMs serve as a docking site for spleen tyrosine kinase (Syk) via interaction with Src homology 2 (SH2) binding domain (Johnson et al., 1995). Integrins form diffusion barriers around the phagocytic cup that block phosphatases like CD45 from dephosphorylating the ITAM and dampening the signal (Freeman et al., 2016). Activation of Syk leads to the recruitment of specific downstream activators or adaptor proteins. The linker of activated T cells (LAT), an adaptor, is recruited to activated Syk and is responsible for recruitment of PLC $\gamma$ and another adaptor, Grb2 (Tridandapani et al., 2000). Grb2 further recruits Gab2, which along with Syk recruit class I PI3K (PI3KCI) to generate PtdIns $(3,4,5) P_{3}(\mathrm{Gu}$, Botelho, Yu, Grinstein, \& Neel, 
2003). Recruitment of PLC $\gamma$ and PI3KCI illustrate the importance of phosphoinositide signaling during phagocytosis.

\subsubsection{Phosphoinositide dynamics during phagocytosis}

PtdInsPs at the plasma membrane play significant roles for the coordination of actin polymerization during phagocytosis. Coordination of $\operatorname{Ptd} \operatorname{Ins}(4,5) P_{2}$ and $\operatorname{Ptd} \operatorname{Ins}(3,4,5) P_{3}$ levels promote actin assembly and disassembly at specific times during the internalization process. Upon Fc $\gamma$ receptor engagement, PtdIns $(4,5) P_{2}$ levels appear to increase through recruitment of the PtdIns(4)P 5-kinase PIPKI $\alpha$ (Botelho et al., 2000; Mao et al., 2009). To further support synthesis of PtdIns $(4,5) P_{2}$, PLD is necessary for phagocytosis and appears to recruit and activate PIP5K (Divecha et al., 2000; Kusner, Hall, \& Jackson, 1999). Local synthesis of PtdIns(4,5)P $P_{2}$ induces actin polymerization and pseudopod extension around the particle (Scott et al., 2005). As pseudopods extend, PtdIns $(4,5) P_{2}$ synthesis follows pseudopod extension, with a concurrent loss of PtdIns(4,5) $P_{2}$ at the base of the phagocytic cup (Scott et al., 2005). Loss of PtdIns(4,5) $P_{2}$ and actin disassembly is required for phagosome closure (Fairn et al., 2009; Scott et al., 2005) and appears to be dependent on the recruitment of phospholipase $\mathrm{C} \gamma$ (PLC $\gamma$ ) to LAT (Tridandapani et al., 2000).

Alternatively, PtdIns $(4,5) P_{2}$ can also be turned over into PtdIns(3,4,5)P $P_{3}$. Recruitment of the regulatory subunit of PI3KCI, p85, is mediated by SH2 interaction with Syk and Gab2 (Gu et al., 2003; Moon et al., 2005). Not only does PI3KCI deplete PtdIns(4,5) $P_{2}$ from the phagocytic cup, $\operatorname{Ptd} \operatorname{Ins}(3,4,5) P_{3}$ also appears to be necessary for phagocytosis, particularly for larger particles (Schlam et al., 2015; Vieira et al., 2001). One possibility is that PtdIns(3,4,5) $P_{3}$ recruits myosin X, which may be necessary for phagosome closure (Dianne Cox et al., 2002). 
Furthermore, the spatiotemporal generation of $\operatorname{PtdIns}(4,5) P_{2}$ or $\operatorname{PtdIns}(3,4,5) P_{3}$ are necessary to regulate the Rho GTPases at distinct stages of internalization.

\subsubsection{Actin polymerization via Rho GTPases}

Key molecular switches that control actin dynamics are the Rho GTPases. These small GTPases are part of a larger superfamily of Ras GTPases, that include the Rab GTPases necessary for membrane trafficking (described below) and Rag GTPases involved in recruitment of mTORC1 and TFEB to the lysosome. Typically, these GTPases switch between an inactive GDP-bound form and an active GTP-bound form. One of the exceptions Rag C/D that is considered active when bound to GDP. Nevertheless, GDP-bound GTPases are considered off due to their inability to bind to effector proteins. While GDP-bound, some GTPases can interact with guanine dissociation inhibitors (GDI), which inhibits activation by GEFs and may mask the membrane-anchoring prenyl group (Dransart, Morin, Cherfils, \& Olofsson, 2005). To become active, GDP is removed by guanine nucleotide exchange factors (GEFs) and replaced with GTP. GTPases with prenyl groups may be deposited into their target membranes. There, the active GTPase is now in a conformation that allow binding to effectors. Although GTPases have hydrolytic activity towards GTP, their enzymatic activity is slow and inefficient. GTPase activating proteins (GAPs) help to induce hydrolysis of the $\gamma$-phosphate of GTP, returning the GTPase to an inactive state.

The GTPases involved in actin polymerization during Fc $\gamma$ receptor-mediated phagocytosis include those of the Rho family: Cdc42, Rac1 and Rac2. Cdc42 is activated first to initiate actin polymerization, with Rac1 following shortly thereafter and then Rac2 (Hoppe, 2004). Interestingly, Rac2 only becomes active at the phagocytic cup (Hoppe, 2004). Activation 
of Cdc42 depends on recruitment of the adaptor Nck, which can be phosphorylated by Src family kinases (Dart, Donnelly, Holden, Way, \& Caron, 2012). This in turn recruits the Cdc42 GEF intersectin-1, Cdc42 itself, and nucleation promotion factor (NPF) Wiskott-Aldrich Syndrome protein (WASp) to promote actin polymerization (Humphries, Donnelly, \& Way, 2014). Additionally, WASp is stabilized at the plasma membrane by the presence of $\operatorname{PtdIns}(4,5) P_{2}$ (Higgs \& Pollard, 2000; Pauker et al., 2014). In contrast, activation of Rac1/2 is thought to be mediated by the GEFs Dock180-ELMO and Vav. Activated ITAMs recruit the adaptor protein CrkII, which in turn recruits the Rac-GEF Dock180-ELMO (Lee, Cosio, Ireton, \& Grinstein, 2007). On the other hand, Vav may be directly activated by Syk (Deckert, Tartare-Deckert, Couture, Mustelin, \& Altman, 1996; Hall et al., 2006). Rac1, together with PtdIns(4,5) $P_{2}$ and Nck1, can activate N-WASp to promote actin polymerization (Tomasevic et al., 2007).

Together, activated WASp and N-WASp interact with the Arp2/3 complex, which leads to branched-actin polymerization (May, Caron, Hall, \& Machesky, 2000). Arp2/3 is a sevensubunit complex, featuring the actin-like Arp2 and Arp3. Activation of WASp by Rho-GTPases relives autoinhibition exposing two domains necessary for actin assembly (A. S. Kim, Kakalis, Abdul-Manan, Liu, \& Rosen, 2000). The C-terminal VCA domain of WASp interacts with Arp2/3, inducing a conformational change, while the N-terminal Wiskott-Aldrich syndrome protein homology 2 (WH-2) domain binds and delivers actin to Arp2/3 (Machesky \& Insall, 1998). Together, Arp $2 / 3$ and the G-actin monomer form a trimer that can nucleate actin polymerization from an existing actin filament. As actin polymerizes and pushes the plasma membrane, additional $\mathrm{Fc} \gamma$ receptors engage the particle and promote zippering of the plasma membrane around the particle (Swanson \& Baer, 1995). 
As described above, phagosome closure requires actin depolymerization and PtdIns(4,5) $P_{2}$ turnover. PtdIns $(4,5) P_{2}$ must be depleted from the membrane to stop Cdc42 and Rac1-mediated WASp/N-WASp activation. PtdIns $(4,5) P_{2}$ can be turned over by PLC $\gamma$, which forms diacylglycerol (DAG) and inositol trisphosphate ( $\left.\mathrm{IP}_{3}\right)$ (Tridandapani et al., 2000). Alternatively, PtdIns $(3,4,5) P_{3}$ generation by PI3KCI is required to recruit GAPs for Cdc42 and Rac1 (Schlam et al., 2015). As PtdIns(4,5) $P_{2}$ is consumed and Rho GTPases are deactivated, actin depolymerizes from the phagocytic cup. Subsequent closure of the phagosome may be mediated by myosin X (Dianne Cox et al., 2002).

\subsubsection{Phagosome maturation}

Immediately following phagocytic cup closure, the particle encapsulated in a nascent phagosome that resembles the plasma membrane and extracellular milieu. This phagosome is illequipped to digest the particle and must undergo maturation to acquire degradative properties. Phagosome maturation involves step-wise fusion with early endosomes, late endosomes, and lysosomes, a process that largely mirrors the endocytic pathway. Fusion of phagosomes with early endosomes requires the recruitment of the Rab5 GTPase (Vieira et al., 2003). Rab5 recruitment is mediated by the presence of Rab20 at the phagosome, which recruits the Rab5 GEF Rabex-5 (Pei, Repnik, Griffiths, \& Gutierrez, 2014). GTP-bound Rab5 enhances its own activation by recruiting Radaptin-5, which activates Rabex-5, promoting a positive-feedback loop to promote Rab5 activity (Stenmark, Vitale, Ullrich, \& Zerial, 1995; Z. Zhang et al., 2014). Activated Rab5 recruits additional effectors to promote fusion with the early endosome. A key step is the acquisition of PtdIns(3)P at the phagosome, which depends on indirect recruitment of Vps34 by Rab5. By interacting with p150 of the Vps34 complex, Rab5 recruits the class III 
PI3K, enriching the phagosome with PtdIns(3)P. Next, EEA1 interacts with both PtdIns(3)P and Rab5 to facilitate fusion between early endosomes and phagosomes by coordinating the early endosomal soluble N-ethylmaleimide-sensitive factor attachment protein receptors (SNAREs) syntaxin-13 and potentially syntaxin-6 (Christoforidis et al., 1999; Collins, Schreiber, Grinstein, \& Trimble, 2002; Lawe et al., 2002; McBride et al., 1999; Simonsen, Gaullier, D’Arrigo, \& Stenmark, 1999).

Further maturation involves Rab switching from Rab5 to Rab7. The Mon1, complexed with Ccz1, is recruited to GTP-bound Rab5 and has two major functions (Poteryaev, Datta, Ackema, Zerial, \& Spang, 2010). First, Rabex-5 is displaced terminating the positive feedback loop of Rab5 activation (Poteryaev et al., 2010). Second, Rab7 is recruited to the phagosome where Mon1-Ccz1 acts as the GEF for Rab7 activation (Cabrera et al., 2014; Kinchen \& Ravichandran, 2010; Nordmann et al., 2010; Yasuda et al., 2016). Rab7 is essential for phagosome maturation but the proceeding steps leading to lysosome fusion are less clearly defined (Harrison, Bucci, Vieira, Schroer, \& Grinstein, 2003).

Evidence suggests Rab7 is necessary for several roles in maturation. Transport of the phagosome towards the lysosome is mediated by the microtubules and the minus-end directed motor protein complex dynein-dynactin (Johansson et al., 2007). Rab7 interacts with the adaptor Rab7-interacting lysosomal protein and oxysterol-binding protein-related protein 1L, which in turn interact with dynein-dynactin (Harrison et al., 2003; Johansson et al., 2007). Association with RILP and dynein-dynactin may also be necessary for phagosome membrane tabulation towards the lysosome for the direct acquisition of the V-ATPase pump (Harrison et al., 2003; Sun-Wada, Tabata, Kawamura, Aoyama, \& Wada, 2009). Fusion with of the phagosome with late endosomes may be facilitated by the HOPS complex, which features two Rab7 binding 
subunits. Similar to EEA1 above, HOPs may also coordinate SNAREs, including SNAP-23 and syntaxin 7 (Collins et al., 2002; Sakurai et al., 2012).

Another key determinant in phagosome maturation is the turnover of $\operatorname{PtdIns}(3) P$ to PtdIns(3,5) $P_{2}$. Independently of Rab switching, PtdIns(3) $P$ appears to be synthesized until the $\mathrm{pH}$ of the phagosome decreases, inducing Vps34 inactivation and turnover of PtdIns(3)P to PtdIns(3,5) $P_{2}$ (Naufer et al., 2018). PtdIns(3,5) $P_{2}$, also appears to be necessary for phagosomelysosome fusion. Disrupting PtdIns(3,5) $P_{2}$ synthesis lead to delayed acquisition of lysosomal markers and reduced degradation (G. H. E. Kim, Dayam, Prashar, Terebiznik, \& Botelho, 2014). This effect is in part due to reduced activity of the PtdIns $(3,5) P_{2}$-gated calcium channel, TRPML1. TRPML1 silencing or chelation of calcium lead to phagosomes docked to lysosomes that were unable to fuse (Dayam, Saric, Shilliday, \& Botelho, 2015). Once phagosomes fully fuse with the lysosomes, the particle is fully degraded by the various hydrolases and acidic lumen.

\subsubsection{Rationale and hypothesis}

Lysosome biogenesis during starvation enhances degradation of autophagosomes to liberate energy to enhance survival (Roczniak-Ferguson et al., 2012). Similarly, phagocytes may use the same strategy in response to pathogens to be degraded. Making more lysosomes may enhance degradation and prepare the immune system for subsequent waves of pathogens. We hypothesize that macrophages challenged with IgG-opsonized latex beads will enhance lysosome biogenesis, priming the cell to be more proteolytic.

To test this, we focus on three main objectives:

1) To determine if Fc $\gamma$ receptor engagement is sufficient for TFEB activation; 
2) To determine whether Fc $\gamma$ receptor engagement enhances proteolytic potential of the macrophage;

3) To elucidate the signaling pathway that connects the Fc $\gamma$ receptor with TFEB. 


\subsection{Figures}


A

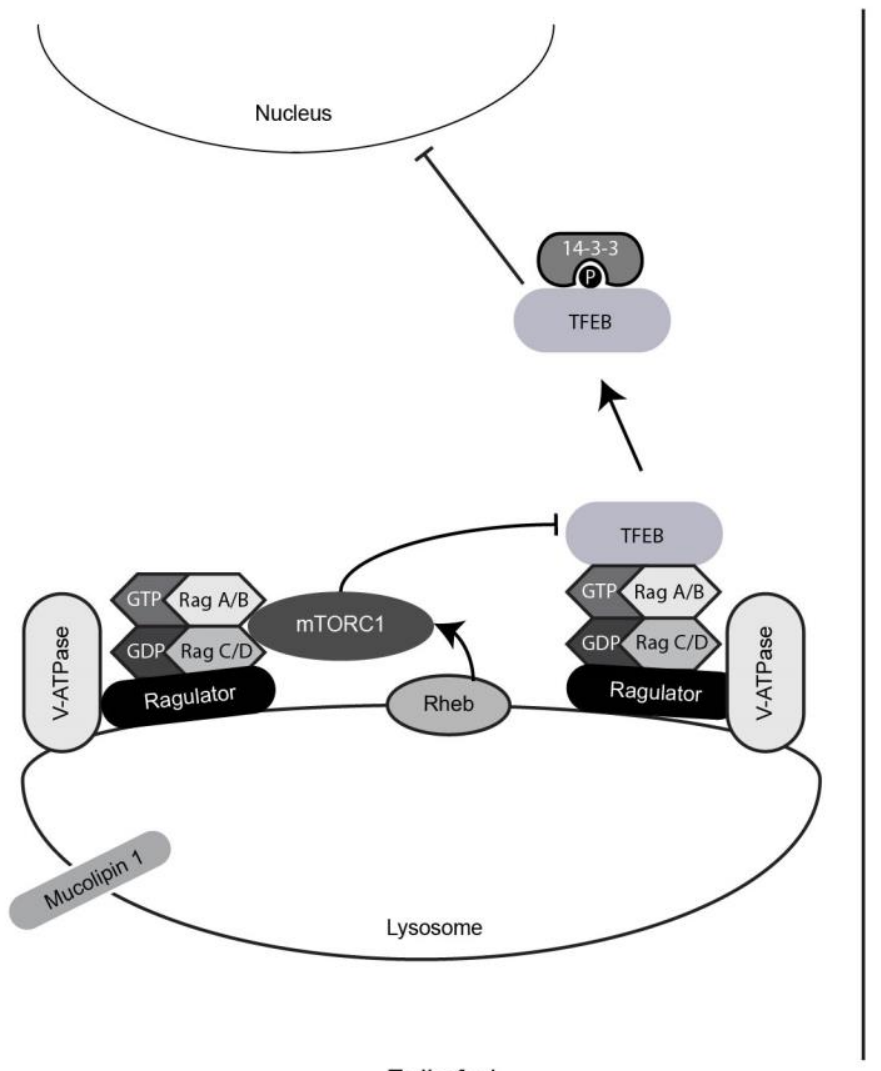

Fully fed
B

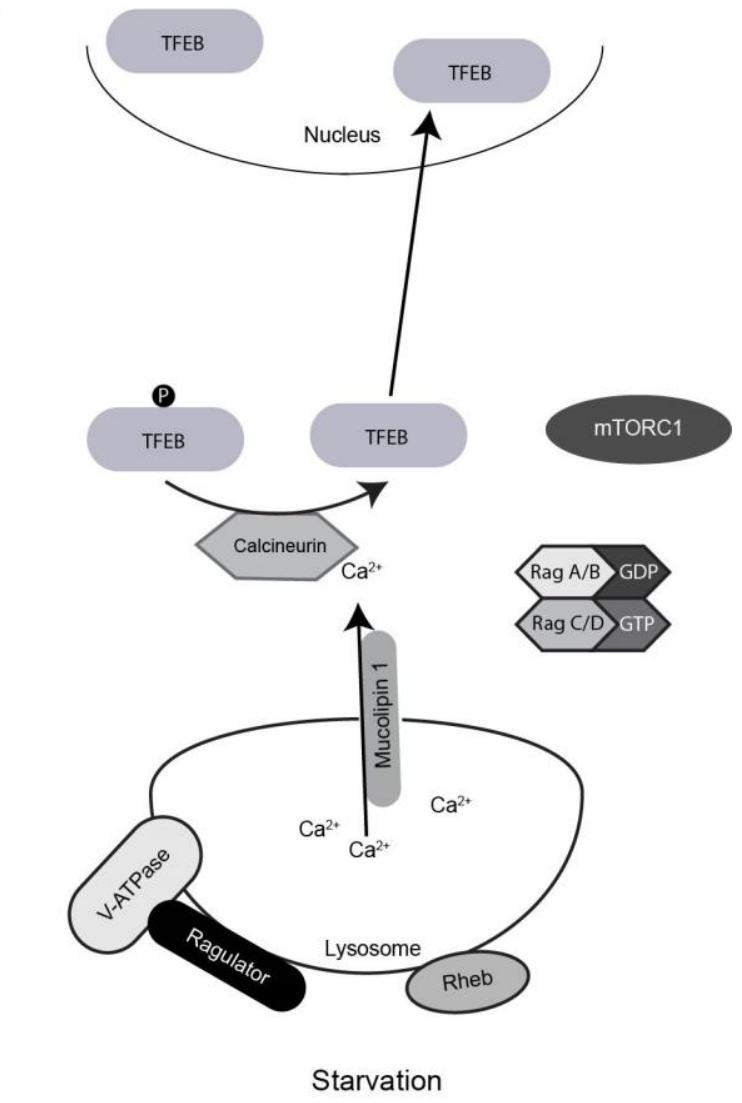


Figure 1.1.1: TFEB regulation by mTORC1 and nutrients. The best understood regulator of TFEB is mTORC1 in response to nutrients. (A) When amino acids are available, Rag GTPases recruit mTORC1 and TFEB to the lysosomal surface. mTORC1 is further activated by Rheb in response to growth factors, and phosphorylate TFEB on S211, among others. Phosphorylated TFEB associates with 14-3-3, which blocks the nuclear localization sequence, leading to cytosolic sequestration. (B) Under starvation conditions, Rag GTPases are off and mTORC1 is inhibited. Here, the calcium channel mucolipin-1 becomes active and releases lysosomal calcium to promote calcineurin activity. This phosphatase dephosphorylates TFEB at S211, allowing TFEB to enter the nucleus. 


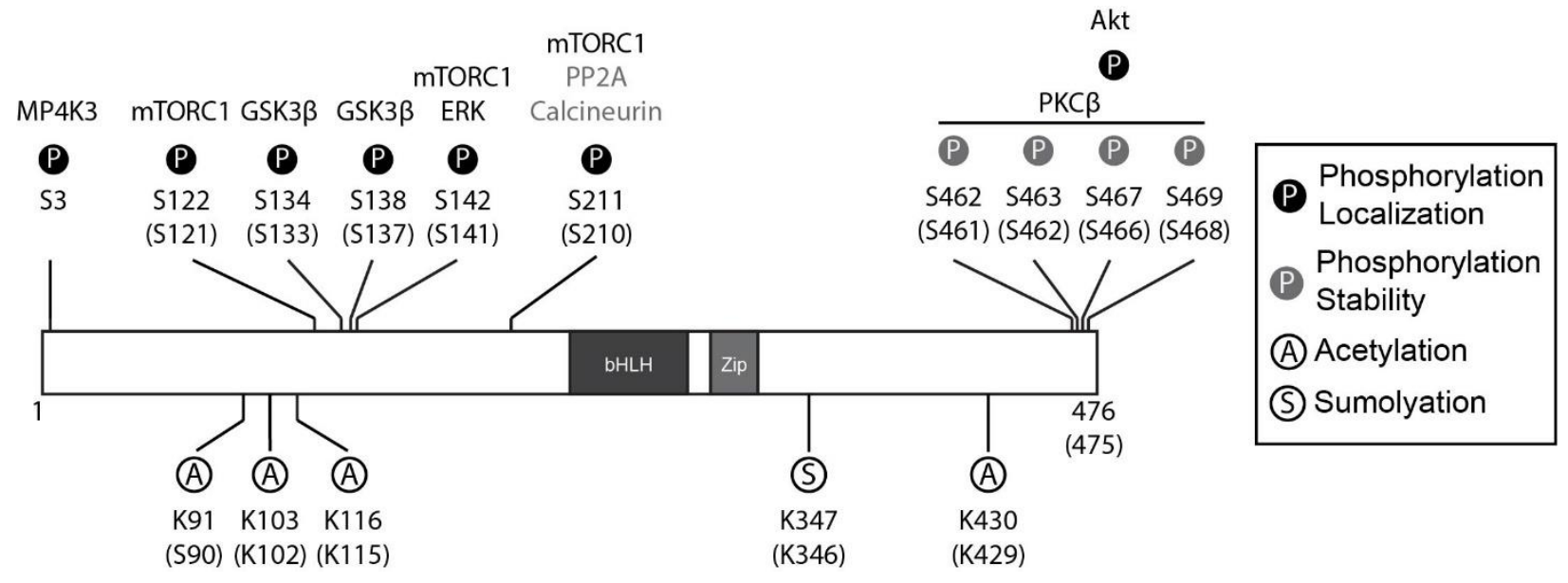


Figure 1.1.2: Regulation of TFEB by post-translational modifications. TFEB is known to be phosphorylated by at six kinases and two phosphatases. Many of these phosphates modulate nuclear/cytosolic localization (black circles with P). PKC $\beta$ appears to be unique, modulating TFEB stability instead (grey circles with P). TFEB is also known to be modified with SUMO (white circle with S) and acetyl (white circle with A) groups, altering their DNA binding properties. The relative positions and residues are indicated along the protein. Indicated in parentheses is the residue numbering in mouse, if different from human. 
A
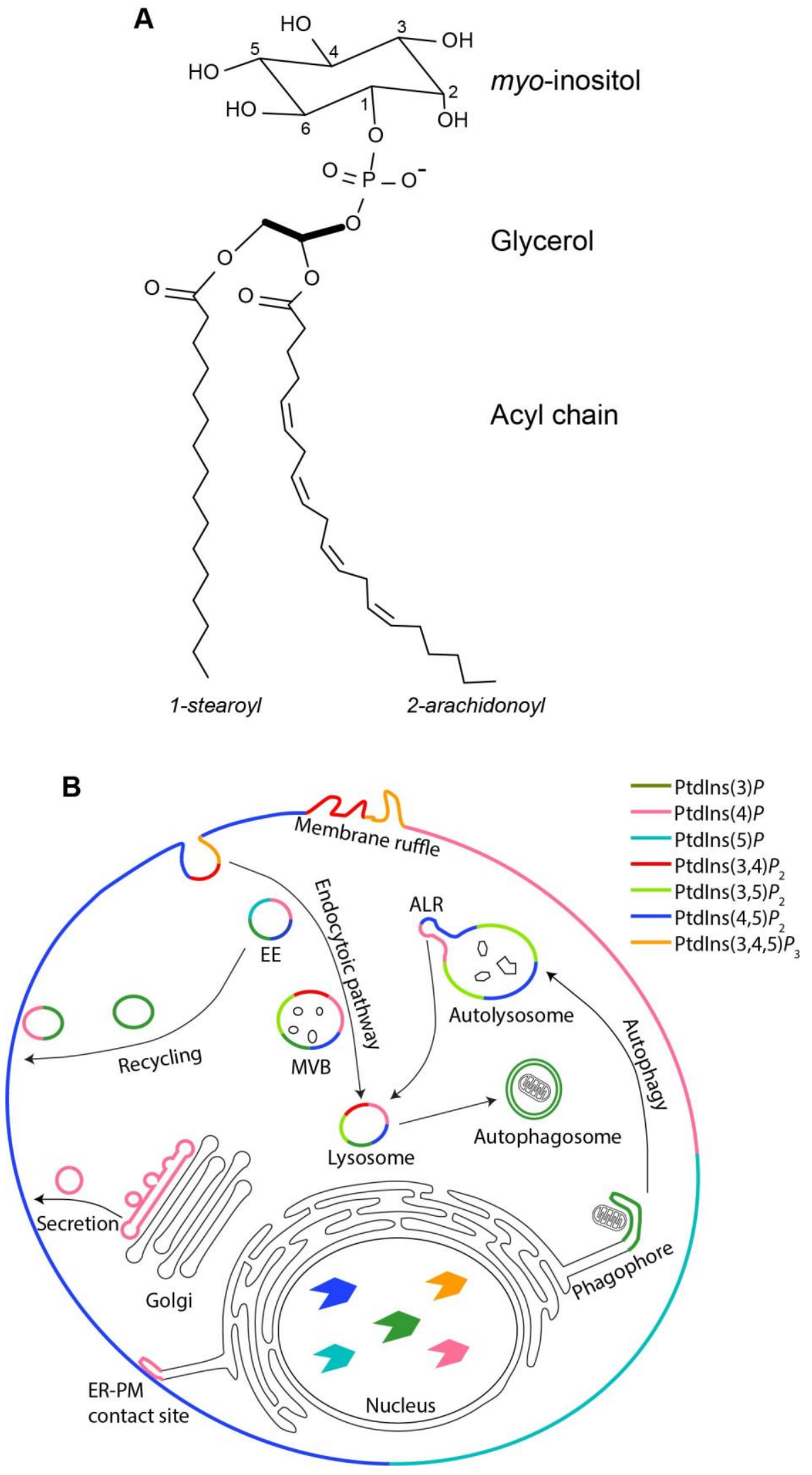
Figure 1.2.1: Phosphatidylinositol structure and localization of its phosphorylated derivatives. (A) Phosphatidylinositol is composed of a glycerol backbone, a myo-inositol head group, and two fatty acid tails. These fatty acids are most commonly 1-stearyol-2-aracadonl.

Phosphorylation of the headgroup can occur at positions 3 and/or 4 and/or 5, yielding 7 phosphorylated derivatives or PtdInsPs. (B) These PtdInsPs are typically thought to have very narrow localization throughout the cell. However, each organelle typically hosts multiple PtdInsPs, which can be generated in response to a specific stimulus or accumulate to regulate basal function of the organelle. This figure is published in (Choy, Han, \& Botelho, 2017). 


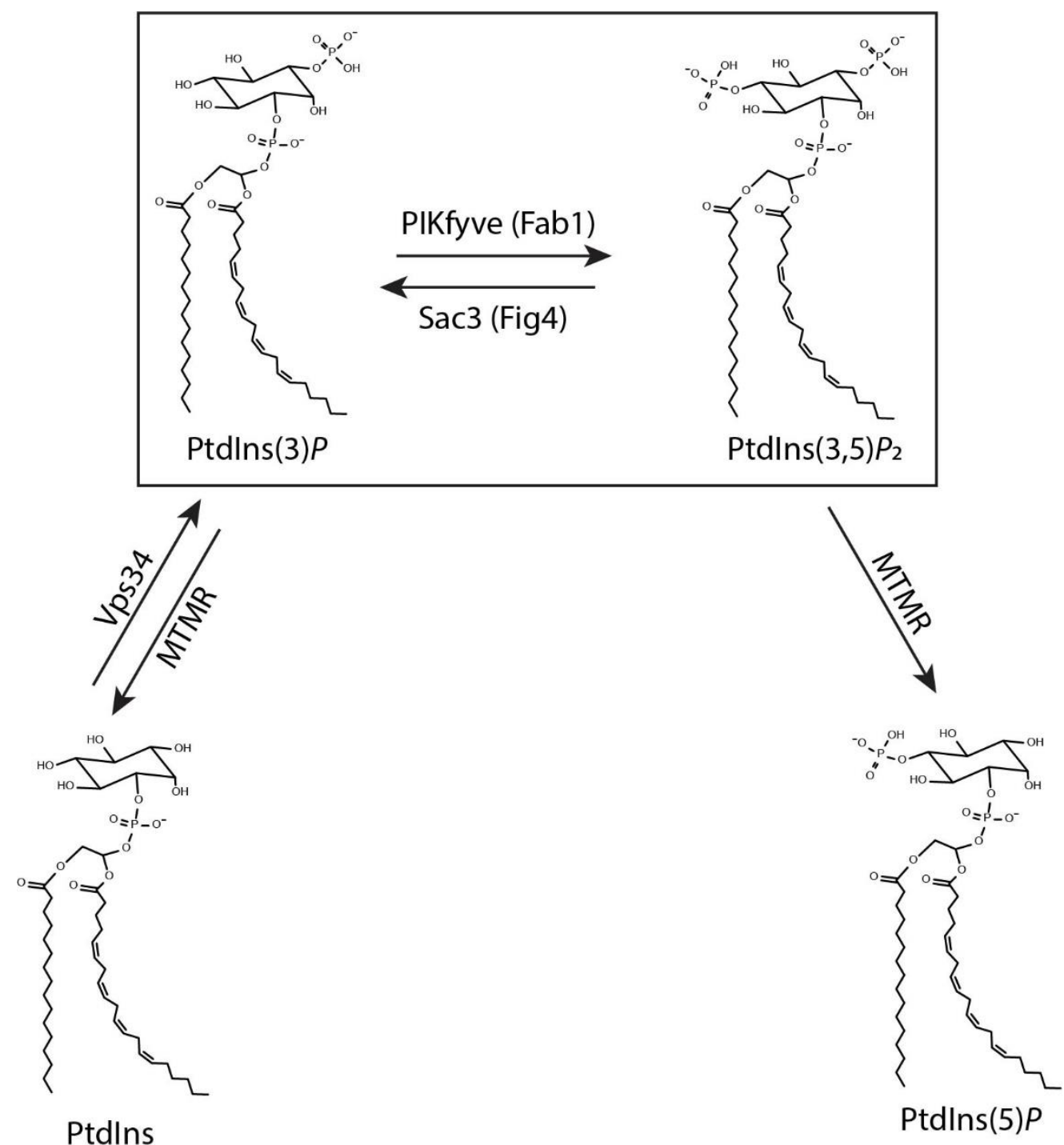


Figure 1.2.2: Synthesis and turnover of $\operatorname{PtdIns}(3,5) P_{2}$. The conversion of PtdInsP is often mediated by numerous kinases and phosphatases. However, $\operatorname{Ptd} \operatorname{Ins}(3,5) P_{2}$ is thought to be synthesized by a single pathway. Endosomal PtdIns is phosphorylated by Vps34 to generate PtdIns(3) $P$. This PtdIns(3) $P$ is the primary precursor for PtdIns(3,5) $P_{2}$ and is phosphorylated by the PIKfyve kinase. PtdIns(3,5) $P_{2}$ can be turned over by the 5-phosphatase Sac3 into PtdIns(3)P, or by 3-phosphatases MTMR to yield PtdIns(5)P. 


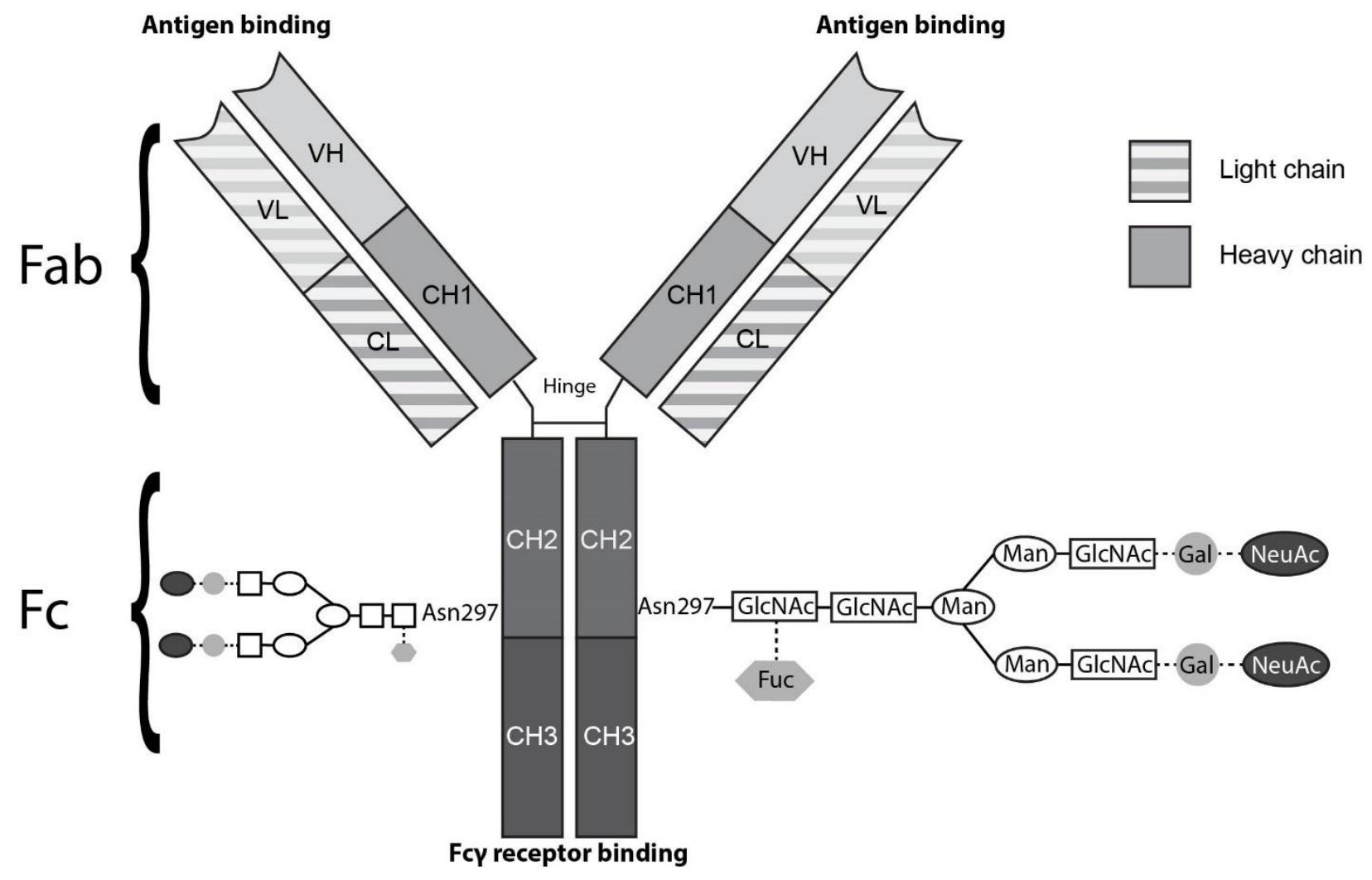


Figure 1.3.1: Basic structure of typical immunoglobulin G. IgG is made up of two heavy chains and two light chains. The heavy chain is made up of 4 regions, one variable region (VH) and three constant regions (CH1-3). Meanwhile, the light chain is made up of two regions, one variable (VL) and one constant (CL) region. The variable regions form heterodimers and facilitate antigen binding (Fab region). In contrast, the $\mathrm{CH} 2-\mathrm{CH} 3$ domains of the two heavy chains dimerize and are responsible for Fc $\gamma$ receptor binding. A critical feature of the $\mathrm{CH} 2$ domain is the presence of a glycan chain at Asn297 that is required for Fc $\gamma$ receptor binding. Different IgG isoforms often differ in their hinge region and can bind to Fc $\gamma$ receptor isoforms with different affinities. 


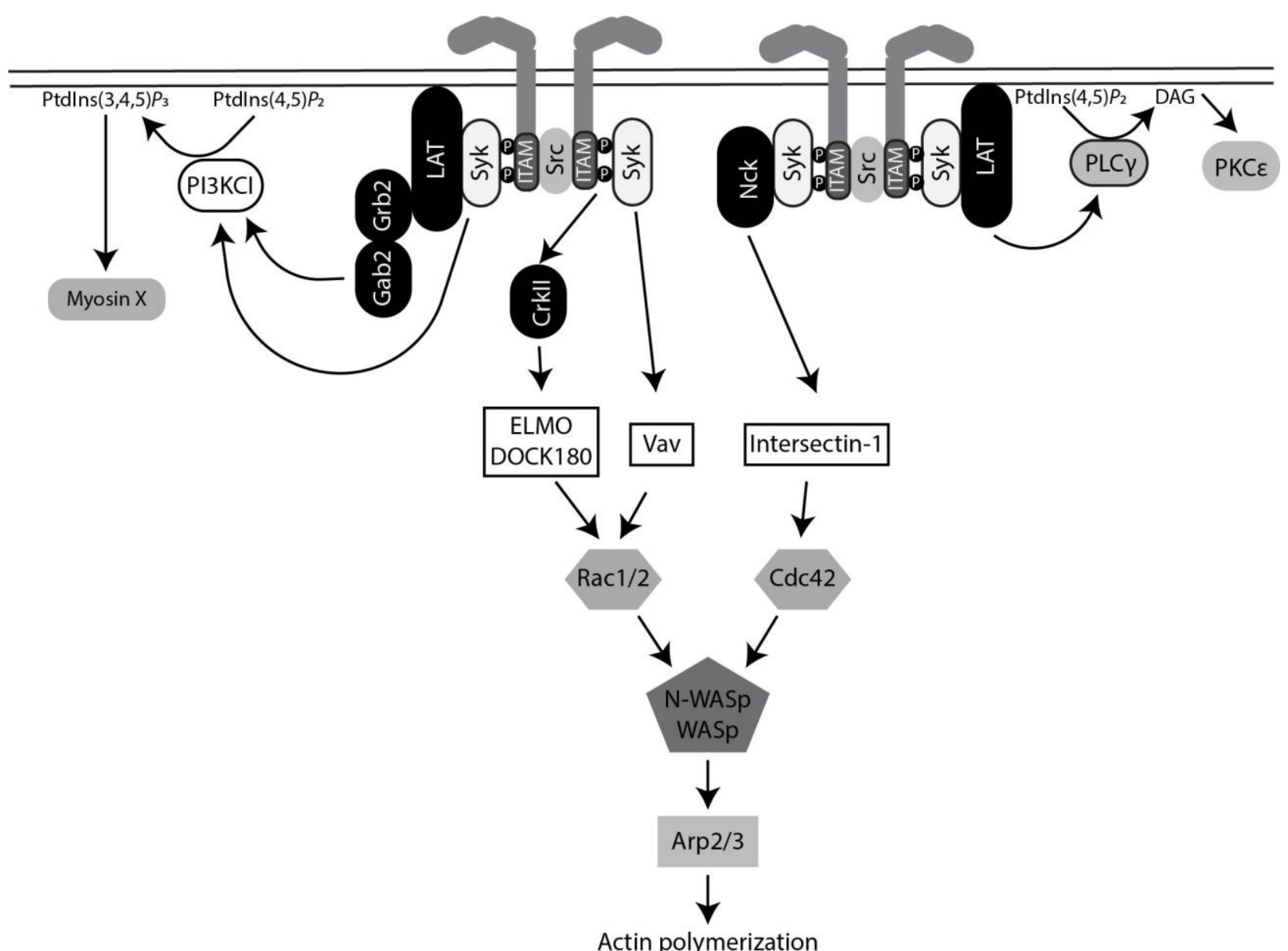


Figure 1.3.2: Fc $\gamma$ receptor signaling. Activation of Fc $\gamma$ receptor by multivalent IgG leads receptor clustering and activation of ITAM domains by Src-family kinases. Phosphorylation of the ITAM recruits Syk, followed by numerous adaptor proteins and other signaling mediators. The combined effect of various lipid and protein kinases, GTPases, and actin nucleators is the actindependent extension of pseudopods around the particle leading to particle internalization. 
Chapter 2: Materials and methods 


\section{$\underline{2.1 \text { Cell culture and transfection }}$}

RAW 264.7 murine macrophages were purchased from ATCC (Manassas, VA). RAW macrophages with CRISPR knockouts of TFEB, TFE3, or TFEB and TFE3 were gifts provided by Dr. Rosa Puertollano at the National Institutes of Health (Pastore et al., 2016). HeLa cells were purchased from ATCC. All cell lines were cultured in the presence of Dulbecco's Modified Eagle's Medium (DMEM; Wisent, St. Bruno, QC or Gibco, Waltham, MA), supplemented with 5-10\% fetal bovine serum (Wisent or Gibco), at $37^{\circ} \mathrm{C}$ and $5 \% \mathrm{CO}_{2}$.

Plasmids encoding TFEB-GFP, TFE3-GFP, and MiTF-GFP were gifts provided by Dr. Shawn Ferguson at Yale University (Roczniak-Ferguson et al., 2012). cDNA plasmids were introduced into RAW cells using FuGENE HD (Promega, Madison, WI) following manufacturer's guidelines at a 3.5:1 ratio of FuGENE HD to DNA. Briefly, $1.1 \mu \mathrm{g}$ of DNA was diluted in water or serum-free DMEM to a concentration of $20 \mu \mathrm{g} / \mathrm{mL}$ and complexed with 3.9 $\mu \mathrm{L}$ of FuGENE HD. The transfection complex was mixed and incubated for $10 \mathrm{~min}$ before addition to RAW macrophages per $1 \mathrm{~mL}$ of complete media. Transfection reagents were washed off after $4 \mathrm{~h}$ and replaced with complete media. Experiments were performed $24 \mathrm{~h}$ posttransfection.

Gene silencing was performed against Rab7 with individual siRNA oligonucleotides (Dharmachon, Lafayette, CO) by electroporation using the Neon transfection system (Invitrogen, Waltham, MA). Briefly, $\sim 6 \times 10^{6} \mathrm{RAW}$ cells were grown to confluence in T-25 flasks. Cells were washed with in PBS, scraped and pelleted at $500 \mathrm{x} g$ for $5 \mathrm{~min}$. The cell pellet was resuspended in resuspension buffer $\mathrm{R}$ at a ratio of $100 \mu \mathrm{L}$ per $1 \times 10^{6}$ cells and $200 \mathrm{nM}$ of siRNA was added. The following oligonucleotides against mouse Rab7a (gene ID: 19349) were used: oligo 1 (open reading frame [ORF] J-040859-17-0002) or oligo 2 (ORF J-040859-18-0002). Cells were then 
electroporated in $100 \mathrm{~mL}$ volumes with 1 pulse at $1730 \mathrm{~V}$ for $20 \mathrm{~ms}$. After $24 \mathrm{hr}$, cells were subjected to an additional round of knockdown and experiments were performed $48 \mathrm{~h}$ after initial knockdown.

\section{$\underline{2.2 \text { PIKfyve inhibition }}$}

Cells were treated with apilimod (20 nM unless otherwise stated, Toronto Research Chemicals, Toronto, ON) at indicated times. Torin1 (100 nM, Tocris Bioscience, Minneapolis, $\mathrm{MN}$ ) was used as a positive control of TFEB nuclear localization for $1 \mathrm{~h}$, unless otherwise stated.

\subsection{Phagocytosis and endocytosis}

IgG opsonized beads were prepared by incubating $3.0 \mathrm{mg} / \mathrm{mL}$ of $\mathrm{IgG}$ from human sera (Sigma Aldridge, St. Louis, MO) with PBS in the presence of $15.0 \mathrm{mg} / \mathrm{mL}$ of beads. Depending on the application, $3.0 \mu \mathrm{m}$ polystyrene beads (Sigma Aldridge), $3.87 \mu \mathrm{m}$ or $21.25 \mu \mathrm{m}$ crosslinked poly(styrene/divinylbenzene) beads (Bangs laboratories, Fishers, IN) were used. Beads were opsonized by rotating end-over-end for $1 \mathrm{~h}$ at room temperature or overnight at $4^{\circ} \mathrm{C}$. Opsonized beads were briefly spun down and the supernatant was discarded. The bead pellet was washed with $1 \mathrm{~mL}$ PBS and spun again. The final pellet was resuspended in $50 \mu \mathrm{L}$ of PBS. For imaging purposes, $5 \mu \mathrm{L}$ of beads per $1 \mathrm{~mL}$ of complete DMEM was added to the cells, while $50 \mu \mathrm{L}$ of beads per $1 \mathrm{~mL}$ of complete DMEM was added for Western blotting.

Escherichia coli strain DH5- $\alpha$ was prepared by inoculating single colonies into liquid LB, supplemented with ampicillin $(100 \mu \mathrm{g} / \mathrm{mL})$ where necessary. The culture was incubated overnight at $37^{\circ} \mathrm{C}$ at $200 \mathrm{rpm}$ overnight. Overnight cultures pelleted, washed with PBS, and 
resuspended in PBS. E. coli was added to cells at a concentration of 0.06 OD per mL of complete DMEM for imaging or 0.6 OD per mL of complete DMEM for Western blot.

Inhibitors of Fc $\gamma$ receptor signaling were added 10-30 min prior to addition of IgG-

opsonized beads. Cells were inhibited with U-73122 (2 $\mu \mathrm{M}$; U6756; Sigma Aldridge), dasatinib

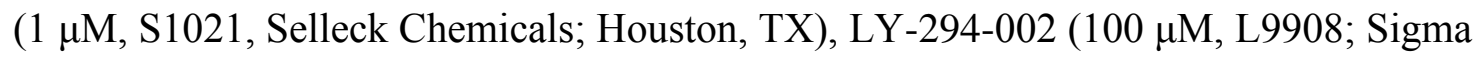
Aldridge), U0126 (10 $\mu \mathrm{M}$, Cell Signaling Technologies, Danvers, MA), cytochalisin D (10 $\mu \mathrm{M}$, EMD Millipore, Burlington, MA), ZSTK474 (1 $\mu \mathrm{M}$; S1072; Selleck). Inhibitors were present in the cell culture media throughout the experiment.

Phagocytic particles were parachuted onto RAW cells at $500 \mathrm{x} g$ for $5 \mathrm{~min}$ and allowed internalize for $30 \mathrm{~min}$ at $37^{\circ} \mathrm{C}$ with $5 \% \mathrm{CO}_{2}$. Uninternalized particles were aspirated and fresh, complete media was added. The internalized particles were allowed to undergo maturation for the times indicated. The reactions were stopped by the addition of ice cold $4 \%$ PFA for imaging or 2x Laemmli sample buffer for Western blotting.

Heat-aggregated $\mathrm{IgG}$ were generated by heating $1.67 \mathrm{mg} / \mathrm{mL} \mathrm{IgG} \mathrm{in} \mathrm{PBS} \mathrm{at} 62^{\circ} \mathrm{C}$ for 20 min. Large aggregates were pelleted at $15,000 \times \mathrm{g}$ for $10 \mathrm{~min}$ and the supernatant was collected. Aggregated IgG was added to cells to a final concentration of $0.2 \mathrm{mg} / \mathrm{mL}$ of complete DMEM and endocytosed for $20-30 \mathrm{~min}$ at $37^{\circ} \mathrm{C}$ and $5 \% \mathrm{CO}_{2}$. External aggregated $\mathrm{IgG}$ was aspirated and fresh DMEM was added to allow for trafficking to the lysosome for $60 \mathrm{~min}$.

Where necessary, torin1 (100 nM) was used as a positive control for TFEB nuclear localization for $1 \mathrm{~h}$, unless otherwise stated. 


\subsection{SDS-PAGE and Western Blot}

Whole cell lysates were generated with 2x Laemmli sample buffer, or 1\% Triton X-100 in PBS and mixed 1:1 with 2x Laemmli sample buffer. Lysates were supplemented with protease inhibitor (Sigma-Aldrich) and/or PhosSTOP (Roche, Basel, Switzerland) where necessary. Lysates were passed through 27-gauge needle until smooth and boiled. Puromycylation assays were performed with the addition of $10 \mu \mathrm{g} / \mathrm{mL}$ of puromycin (EMD Millipore) $15 \mathrm{~min}$ before cell lysis. Samples that required dephosphorylation were lysed in 1\% Triton X-100. Lysates were cleared by centrifugation at $16,000 \times \mathrm{x}$ for $10 \mathrm{~min}$ then subjected to Lambda protein phosphatase (New England Biolabs, Ipswich, MA) as per manufacturer's instruction. Briefly, $20 \mu \mathrm{g}$ of protein was incubated with $50 \mathrm{mM}$ HEPES, $100 \mathrm{mM} \mathrm{NaCl}, 2 \mathrm{mM}$ DTT, 0.01\% Brij 25, $1 \mathrm{mM} \mathrm{MnCl}$, and 8 units $/ \mu \mathrm{L}$ of Lambda phosphatase at $30^{\circ} \mathrm{C}$ for $60 \mathrm{~min}$. The reaction as stopped with $5 \mathrm{x}$ Laemmli sample buffer and heated.

Most samples were resolved by SDS-PAGE though 8-12\% acrylamide resolving gel. To assay for TFEB mobility shift, we used an 8\% acrylamide gel supplemented with $12.5 \mu \mathrm{M}$ Phostag acrylamide (Wako Chemicals U.S.A., Richmond, VA) and $25 \mu \mathrm{M} \mathrm{MnCl}_{2}$. Phos-tag gels were washed three times with $100 \mathrm{mM}$ EDTA for $10 \mathrm{~min}$ each, and once with transfer buffer $(25 \mathrm{mM}$ Tris-HCl, 192 mM glycine, 20\% methanol). Proteins were transferred to PVDF and blocked with $5 \%$ skim milk or $3 \%$ BSA in Tris-buffered saline (1.5 mM NaCl, $500 \mu \mathrm{M}$ Tris, $\mathrm{pH} 7.5)$ with 0.1\% Tween-20 (TBST). Membranes were then incubated with primary and HRP-conjugated secondary antibodies in the presence of the blocking buffer. Proteins were visualized using Clarity Western enhanced chemiluminescence blotting substrates (Bio-Rad Laboratories, Hercules, CA) with a ChemiDoc XRS+ or ChemiDoc Touch imaging system (Bio-Rad). Proteins 
were quantified using Image Lab software (Bio-Rad) by normalizing against loading control and then normalizing against vehicle-treated control samples.

Rabbit monoclonal antibodies used were against cathepsin D (1:1000, GTX62603, GeneTex Inc., Irvine, CA), HSP60 (1:1000, 12165, Cell Signaling Technologies), p70-S6 kinase (1:1000, 2708, Cell Signaling), Rab7 (1:1000, 9367, Cell Signaling), p44/42 MAPK (1:1000, 4695, Cell Signaling), phospho-Thr202/Tyr204 p44/42 MAPK (1:1000, 4377, Cell Signaling), and GSK3 $\alpha / \beta(1: 1000,5676$, Cell Signaling). Rabbit polyclonal antibodies were used against ATP6V1H (1:1000, GTX110778, GeneTex), TFEB (1:2000, A303-673A, Bethyl Laboratories, Montgomery, TX), phospho-Thr389-p70-S6 kinase (1:1000, 9205, Cell Signaling), $\beta$-actin (1:1000, 4967, Cell Signaling), phospho-Ser9 GSK3ß (1:1000, 9336, Cell Signaling), phosphoSer21/9 GSK3 $\alpha / \beta(1: 1000,9331$, Cell Signaling), and phospho-S142 TFEB (1:500, ABE1971-I, EMD Millipore). Rat monoclonal antibodies were used against LAMP1 (1:200, 1D4B, Developmental Studies Hybridoma Bank, Iowa City, IO). Mouse monoclonal antibodies were used against puromycin (1:1000, 540411, EMD Millipore), and p53 (1:1000, 2524, Cell Signaling). Secondary HRP-conjugated antibodies were raised in donkey (1:10000, Bethyl).

\section{$\underline{2.5 \text { Immunofluorescence }}$}

Cells were fixed with $4 \%$ ice cold paraformaldehyde in PBS for $20 \mathrm{~min}$ at room temperature, followed by incubation with $50 \mathrm{mM}$ L-glycine in PBS for $20 \mathrm{~min}$. Cells were then permeabilized and concurrently blocked using $0.2 \%$ Triton X-100 and 2\% BSA in PBS for 60 min. Alternatively, cells were fixed and permeabilized with ice cold methanol for 5 min at $-20^{\circ} \mathrm{C}$, followed by blocking with 2\% BSA in PBS for 60 min. Primary antibodies at the indicated concentrations were applied for $60 \mathrm{~min}$, followed by secondary antibodies. were applied in PBS 
at the concentrations indicated for $60 \mathrm{~min}$, followed by 3 PBS washes for 5 min each. Secondary antibodies were applied for $60 \mathrm{~min}$ at their respective concentrations in PBS, followed by 5 PBS washes for $5 \mathrm{~min}$ each. Where necessary, DAPI (Roche) was applied to stain the nucleus at 5 ng/mL in PBS. Cells were then mounted onto glass slides with Dako fluorescent mounting media (Dako, Santa Clara, CA). Rabbit monoclonal antibodies were used against TFEB (1:250, A303673A, Bethyl). Donkey polyclonal antibodies conjugated to Dylight (1:500, Bethyl) or cyanine (1:1000, Jackson Immunoresearch, West Grove, PA) fluorescent dyes were used as secondary antibodies.

\subsection{Lysosome labelling for live-cell imaging}

Lysosomes for manual counting were treated with inhibitors before microscopy. Cells with fluorescently labelled lysosomes were incubated with $2.5 \mathrm{mg} / \mathrm{mL}$ of Lucifer yellow (Invitrogen) in complete media for $2 \mathrm{~h}$ at $37^{\circ} \mathrm{C}$ and $5 \% \mathrm{CO}_{2}$. Cells were washed with PBS 3 times, and fresh complete media was added to the cells. The fluid phase marker was allowed to traffic to the lysosome for $1 \mathrm{~h}$ prior to application of inhibitors. Following treatment, cells were taken to the microscope and imaged.

\subsection{Epifluorescence and spinning disk confocal microscopy}

Cells transfected with GFP-fused MiT/TFE family members and manual counting of lysosomes were imaged with a Leica DM5000B system and a DFC350FX camera controlled by Leica application suite (Leica Microsystems Inc., Concord, ON). Endogenous TFEB with PIKfyve inhibition in RAW cells were imaged with an Olympus IX83 microscope with a Hamamatsu ORCA-Flash4.0 digital camera controlled by CellSens Dimensions software 
(Olympus Canada Inc., Richmond Hill, ON). These images were processed by deconvolution using a constrained iterative in CellSens Dimensions. All other imaging of live and fixed cells used a Quorum DisKovery spinning disc confocal microscope system with a Leica DMi8 microscope equipped with an Andor Zyla 4.2 Megapixel sCMOS camera or Andor iXON 897 EMCCD camera, controlled by Quorum Wave FC powered by MetaMorph software (Quorum Technologies, Guelf, ON). Live-cell imaging was done using cell-specific serum-free media without phenol red in a $5 \% \mathrm{CO}_{2}$ champers at $37^{\circ} \mathrm{C}$.

\section{$\underline{2.8 \text { Image analysis }}$}

Cells transfected with GFP-fused TFEB, TFE3, or MiTF were imaged and quantified by comparing the mean cytosolic and nuclear fluorescence intensity using ImageJ. Cells with greater signal in the cytosol over the nucleus were considered cytosolic while cells with greater nuclear signal compared to the cytosol were counted as nuclear. Quantifications were expressed as the percentage of cells having greater mean fluorescence intensity in the nucleus compared to the cytosol. Cells stained for endogenous TFEB were quantified by taking the ratio of the mean nuclear fluorescence, as indicated by DAPI counter-stain, over the mean cytosolic fluorescence on ImageJ.

To manually quantify vacuole diameter, vacuoles were measured on ImageJ and defined with a minimum diameter of $1.5 \mu \mathrm{m}$. The number of vacuoles and diameter were scored against the total number to get the mean vacuole number and diameter. To determine the volume and number of fluorescently-labelled lysosomes, Z-stacks were imported into Icy bioimage software, equipped with 3D analysis and split ROI plugin. Regions of interest were drawn around whole cells and using the spot detector tool, bright spots were detected over a dark background with a 
scale of 3 ( 7 pixels) with 140-sensitivity. Spots were automatically split using the split ROI plugin, and volume information was extracted using the 3D analysis plugin. Alternatively, Zstacks were imported into Volocity (Volocity 6.3.0, Perkin Elmer, Waltham, MA). Images were thresholded with a value $2 \mathrm{x}$ the mean background signal, excluding particles less than $0.3 \mu \mathrm{m}^{3}$. The number and volume of lysosomes were averaged over the total number of cells analyzed.

\subsection{Quantitative Real Time Polymerase Chain Reaction}

Total RNA was extracted from RAW cells with GeneJET RNA purification kit (Thermo Fisher Scientific, Waltham, MA) as per manufacturer's instruction. Equal quantities of RNA were reverse transcribed with SuperScript VILO cDNA synthesis kit (Invitrogen) following manufacturer's guidelines. The resulting cDNA was diluted 1:100 and amplified for quantitative PCR on a Step One Plus Real-Time PCR thermal cycler (Applied Biosystems, Waltham, MA) with Step One Software (V2.2.2, Applied Biosystems) in the presence of TaqMan Fast Advanced Master Mix (Invitrogen). The TaqMan gene expression assays (Invitrogen) for the reference gene ABT1 (Mm00803824_m1) and target genes CTSD (Mm00515586_m1), ATP6V1H (Mm00505548_m1), ATP6V1D (Mm00445832_m1), LAMP1 (Mm00495262_m1), and MCOLN1 (Mm00522550_m1) were assayed in technical triplicates. Target gene expression was determined by relative quantification ( $\Delta \Delta \mathrm{Ct}$ method) to ABT1 and the vehicle-treated control sample.

2.10 Phosphoinositide labeling and measurement by HPLC-coupled flow scintillation

RAW cells were cultured for $24 \mathrm{~h}$ in inositol-free DMEM (MP biomedical, Santa Ana, $\mathrm{CA}$ ) with $10 \mu \mathrm{Ci} / \mathrm{mL}$ myo-[2- $\left.{ }^{3} \mathrm{H}(\mathrm{N})\right]$ inositol (Perkin Elmer), 10\% dialyzed FBS (Gibco), $4 \mathrm{mM}$ 
L-glutamine (Sigma Aldrich), and 1x insulin-transferrin-selenium-ethanolamine (Gibco). Cells were then drug treated with $20 \mathrm{nM}$ apilimod or $100 \mathrm{nM}$ torin 1 for $1 \mathrm{hr}$. Reactions were stopped with $600 \mu \mathrm{L}$ of $4.5 \%$ perchloric acid (v/v) on ice for $15 \mathrm{~min}$. The precipitated phospholipids were scraped and pelleted at $12,000 \times \mathrm{g}$ for $10 \mathrm{~min}$ at $4^{\circ} \mathrm{C}$. Pellets were washed with $1 \mathrm{~mL}$ of ice cold $0.1 \mathrm{M}$ EDTA, pH 8.8 and pelleted again. The phospholipid pellet was resuspended in $50 \mu \mathrm{L}$ of water and deacylated in the presence of $500 \mu \mathrm{L}$ of $45.7 \%$ methanol: $10.7 \%$ methylamine: $11.4 \%$ 1-butanol (v/v) for $20 \mathrm{~min}$ at room temperature followed by incubation at $53^{\circ} \mathrm{C}$ for $50 \mathrm{~min}$. Sample were vacuum-dried and washed twice by resuspending in $300 \mu \mathrm{L}$ of water and drying. The final dried pellet was resuspended in $450 \mu \mathrm{L}$ of water. The gro-inositol was extracted in the presence of $300 \mu \mathrm{L}$ of $80 \%$ 1-butanol, $16 \%$ ethyl ether, and $4 \%$ ethyl formate. The samples were vortexed for $5 \mathrm{~min}$, and the layers were separated by centrifugation at 12,000 x $\mathrm{g}$ for $2 \mathrm{~min}$. The bottom, aqueous layer was collected and extracted twice more. The final aqueous layer was vacuum-dried and resuspended in $50 \mu \mathrm{L}$ of water. Equal counts of ${ }^{3} \mathrm{H}$ were separated by HPLC (Agilent Technologies, Santa Clara, CA) through a strong anion exchange 4.6 x 250-mm column (Phenomenex, Torrance, CA or Hihrom, Berkshire, England) with a flow rate of $1 \mathrm{~mL} / \mathrm{min}$ and subjected to a gradient of water (buffer A) and $1 \mathrm{M}\left(\mathrm{NH}_{4}\right)_{2} \mathrm{HPO}_{4}, \mathrm{pH} 3.8$ (adjusted with phosphoric acid) (buffer B) as follows: 0\% B for $5 \mathrm{~min}, 0$ to $2 \% \mathrm{~B}$ for $15 \mathrm{~min}, 2 \% \mathrm{~B}$ for $80 \mathrm{~min}$, 2 to $10 \% \mathrm{~B}$ for $20 \mathrm{~min}, 10 \% \mathrm{~B}$ for $30 \mathrm{~min}, 10$ to $80 \%$ B for $10 \mathrm{~min}, 80 \%$ B for $5 \mathrm{~min}, 80$ to $0 \% \mathrm{~B}$ for 5 min. The radiolabeled eluate was detected by $\beta$-RAM 4 (LabLogic, Brandon, FL) with a 1:2 ratio of eluate to scintillant (LabLogic) and analyzed by Laura 4 software. PtdInsPs were identified by time of elution and each PtdInsP peak was normalized against the parent phosphatidylinositol peak. Relative abundance of PtdInsPs from each treatment condition was then compared against their respective abundance in the vehicle-treated control condition. 


\subsection{Flow Cytometry and DQ-BSA Proteolysis}

Cells were pre-treated with heat-aggregated IgG continuously for the times indicated prior to lysosome labelling. Lysosomes were co-labeled with $2 \mu \mathrm{M}$ fixable Alexa Fluor 647conjugated $10 \mathrm{kDa}$ dextran (Invitrogen) and $10 \mu \mathrm{g} / \mathrm{ml} \mathrm{DQ}$ Green BSA (Invitrogen) for 15 min, followed by a wash with PBS and allowed to traffic to the lysosome for $1 \mathrm{~h}$ in cell culture media. Cells were chilled with ice cold PBS, scraped, and analyzed for whole-cell fluorescence using the BD FACSCalibur flow cytometer (BD Biosciences, Franklin Lakes, NJ), where 10,000 events were counted per sample per condition using the channels for their respective fluorescence profiles. Background signal was determined with non-labeled cells. The DQ-BSA signal was then normalized against dextran specific signal.

\section{$\underline{2.12 \text { Statistical analysis }}$}

All experiments were done at a minimum of three independent biological replicates, unless otherwise stated. Microscopy images were analyzed for at least 30 cells in each replicate. In most cases, Student's t-tests were used to compare a sample against their control condition with statistical significance of $\alpha=0.05$. One-way ANOVA was used for three or more conditions with $\alpha=0.05$. When samples were normalized, multiple t-tests with Bonferroni correction were applied to increase confidence. 
Chapter 3: Results 
$\underline{3.1 \text { TFEB activation through PIKfyve inhibition does not contribute to lysosome enlargement. }}$

\subsubsection{PIKfyve inhibition induces TFEB nuclear translocation.}

PIKfyve is responsible for generating PtdIns(3,5) $P_{2}$, a lysosomal lipid necessary for maintaining many lysosome functions including lysosome size (Ikonomov et al., 2015; Rutherford, 2006; Yamamoto et al., 1995). Loss of PtdIns(3,5) $P_{2}$ leads to enlarged lysosomes, a process currently thought to occur due to endomembrane trafficking defects (Bissig et al., 2017; Dove et al., 2002; Rutherford, 2006). However, increased lysosome biogenesis via TFEB activation had not been considered. We hypothesized that inhibition of PIKfyve would induce TFEB activation and enhanced de novo lysosome biosynthesis that contributes to lysosome enlargement. Specifically, loss of PIKfyve activity may inhibit mTORC1 function, leading to

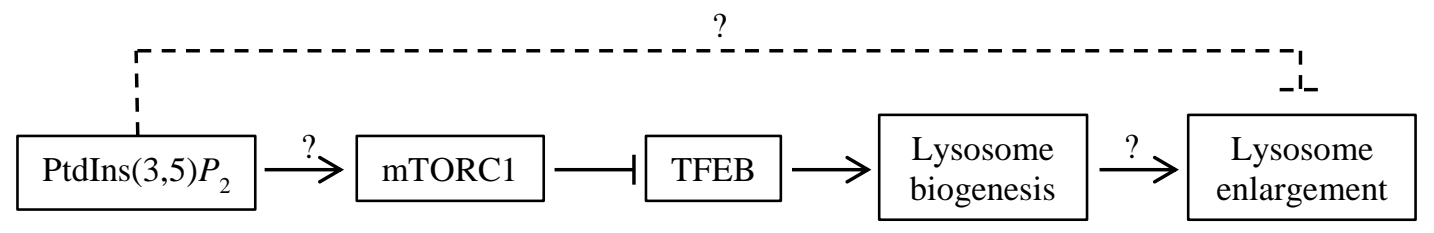

TFEB nuclear localization. To investigate the possible role of TFEB-mediated lysosome biogenesis on during PIKfyve inhibition, we employed the murine RAW 264.7 macrophage cell line. These cells are professional phagocytes and rely on their lysosomes for pathogen clearance (Schlam et al., 2015; Sigola, Fuentes, Millis, Vapenik, \& Murira, 2016). Previous work in our lab showed that inhibition of PIKfyve in RAW cells induces the vacuolation phenotype that can be seen under light microscopy, providing an observable and easily quantifiable phenotype (Ho et al., 2015; G. H. E. Kim et al., 2014).

To deplete PtdIns(3,5) $P_{2}$ in RAW cells, we pharmacologically inhibit the PIKfyve kinase by using apilimod (Figure 3.1.1A). Apilimod was explored as a possible therapeutic for autoimmune diseases, including Crohn's disease and rheumatoid arthritis, where expression of 
the proinflammatory cytokines interleukin-12 (IL-12) and IL-23 are elevated. Use of apilimod reduced IL-12/23 levels in part by binding to the kinase domain of PIKfyve, blocking lipid kinase activity and reducing PtdIns(3,5) $P_{2}$ levels (Cai et al., 2013). In our system, PtdIns(3,5) $P_{2}$ was reduced by $>75 \%$ with $20 \mathrm{nM}$ apilimod for $1 \mathrm{~h}$ (Figure 3.1.1B) (Ho et al., 2015). Concurrently, depletion of PtdIns(3,5) $P_{2}$ lead to enlarged lysosomes that were visible through DIC optics (Figure 3.1.1C) or fluorescently labelled lysosomes (Figure 3.1.1D).

Using these conditions, we tracked TFEB localization in RAW cells by expressing TFEB-GFP or staining for endogenous TFEB. Cells expressing TFEB-GFP increased nuclear localization from $22.5 \pm 7.5 \%$ in control cells to $100 \pm 0 \%$ of cells after $1 \mathrm{~h}$ of apilimod exposure (Figure 3.1.2A). For comparison, we induced TFEB nuclear localization by blocking mTORC1 with torin1, an ATP-competitive inhibitor for mTOR (Thoreen et al., 2009). TFEB-GFP nuclear localization with torin 1 was comparable to apilimod treatment, with $97.0 \pm 3.0 \%$ having nuclear TFEB-GFP (Figure 3.1.2A). Similarly, apilimod treatment increased the nuclear-to-cytosolic ratio of endogenous TFEB to $0.92 \pm 0.03$ compared to $0.55 \pm 0.04$ in vehicle-treated cells (Figure 3.1.2B). Additionally, we checked whether PIKfyve inhibition would induce TFEB nuclear localization in other cell types. In HeLa cells, TFEB nuclear-to-cytosolic ratio also increased from $0.57 \pm 0.09$ in control cells to $1.15 \pm 0.07$ in apilimod-treated cells (Figure 3.1.2C), suggesting apilimod-induced TFEB nuclear localization is not exclusive to macrophages.

Finally, since MiTF and TFE3 appear to cooperate with TFEB in many scenarios (Martina et al., 2014; Ploper et al., 2015), we evaluated their localization in RAW cells. Both TFE3-GFP (Figure 3.1.3A) and MiTF-GFP (Figure 3.1.3B) localized to the nucleus in $>90 \%$ of cells after apilimod treatment compared to $>40 \%$ in vehicle-treated cells. Together, these results 
show that PIKfyve inhibition with apilimod induces nuclear localization of MiT/TFE family members and suggest further cooperativity by these family members.

\subsubsection{PIKfyve inhibition increases dephosphorylation of TFEB}

TFEB nuclear localization is primarily driven by dephosphorylation of key serine residues throughout the protein (Puertollano et al., 2018). Traditionally, others have relied on gel mobility shifts to detect changes in TFEB phosphorylation in whole cell lysates (Puertollano et al., 2018; Roczniak-Ferguson et al., 2012; Settembre et al., 2011), where increased gel mobility is assumed to result from TFEB dephosphorylation. To confirm that TFEB phosphorylation status changed in PIKfyve inhibited cells, we supplemented our SDS-PAGE gels with Phos-tag acrylamide. When complexed with a divalent cation, either $\mathrm{Mn}^{2+}$ or $\mathrm{Zn}^{2+}$, Phos-tag separates proteins by phosphorylation abundance or position with much higher resolution than typical SDS-PAGE, in addition to molecular weight (Kinoshita, Kinoshita-Kikuta, Takiyama, \& Koike, 2006). Therefore, a single band can be resolved into multiple bands based on phosphorylation, with the least phosphorylated species running the fastest.

Running whole cell lysates through these Phos-tag gels resulted in a distinctive banding pattern (Figure 3.1.4, lane 2). To confirm that the antibody is specific for TFEB, we also ran a whole cell lysate from RAW cells knocked out for $t f e b^{-/-}$by CRISPR/Cas9 (Figure 3.1.4, lane 1). Qualitatively, apilimod-treated RAW cells had a distinct TFEB banding pattern relative to the vehicle-treated control (Figure 3.1.4, lane $4 \& 2$ respectively). Interestingly, the pattern observed with apilimod treatment was similar to the torin 1 treatment (Figure 3.1.4, lane $4 \& 3$

respectively). For comparison, we subjected the whole cell lysates to lambda protein phosphatase to dephosphorylate TFEB further (Figure 3.1.4, lane 5, 6 \& 7). In all cases, application of lambda 
phosphatase collapsed all bands into a single, faster running band, suggesting that TFEB retains some phosphorylation with apilimod or torin1.

\subsubsection{TFEB dephosphorylation may result from inhibition of GSK3 but not mTORC1 or ERK2.}

mTORC1 is reported to be recruited and activated by $\operatorname{PtdIns}(3,5) P_{2}$ in yeast and adipocytes (Bridges et al., 2012; N. Jin et al., 2014). Thus, activation of TFEB in PIKfyveinhibited cells may result from inhibition of mTORC1 during PtdIns(3,5) $P_{2}$ depletion. To test this, we evaluated the phosphorylation status of p70-S6K (S6K hereafter) as a reporter for mTORC1 activity. When active, mTORC1 phosphorylates S6K at Thr389 (Pearson et al., 1995). Under resting conditions, cells were fully fed with amino acids and growth factors and S6K is phosphorylated (Figure 3.1.5A, lane 1). When mTORC1 was inhibited with torin1, S6K phosphorylation significantly decreased to $5.6 \pm 4.4 \%$ of control (Figure 3.1.5A, lane 2). In comparison, S6K phosphorylation was not significantly reduced in apilimod-treated cells compared to the vehicle-treated control cells, suggesting that mTORC1 remains active during PIKfyve inhibition (Figure 3.1.5A, lane 3, $4 \& 5$ ). This is consistent with previous reports that PIKfyve inhibition does not block mTORC1 activity (Krishna et al., 2016; W. Wang et al., 2015).

To test the possibility that mTORC1 may act upstream of PIKfyve, we checked the abundance of PtdIns(3,5) $P_{2}$ during mTORC1 inhibition (Figure 3.1.5B). While apilimod-treated

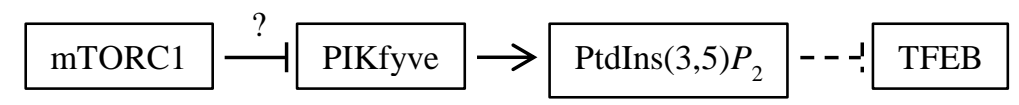

cells displayed a $78 \pm 10 \%$ reduction in $\operatorname{PtdIns}(3,5) P_{2}$, mTORC1 inhibition with torin1 did not reduce PtdIns(3,5) $P_{2}$ levels (Figure 3.1.5B). Instead, the levels of PtdIns(3,5) $P_{2}$ increased to 132 
$\pm 14 \%$ of control. Taken together, TFEB nuclear localization during apilimod treatment does not appear to be a result of mTORC1 inactivation.

Next, we investigated the role of other known kinases that can modulate TFEB phosphorylation. ERK2 was the first kinase suggested to phosphorylate TFEB at S142 and to control cytosolic-nuclear localization (Settembre et al., 2011). To assay ERK2 activity, we probed for phosphorylation of ERK2 (Figure 3.1.6). Activation of ERK2 correlates with phosphorylation levels of ERK2 at T185 and Y187 (Payne et al., 1991). Phosphorylation of ERK2 in control cells is ablated by the ERK specific inhibitor U0126, reducing phosphorylation to $20 \pm 10 \%$ of control (Figure 3.1.6A, lane 2, B). Inhibition of ERK with U0126 also reduced phosphorylation of ERK1 (Figure 3.1.6C). However, there was no significant difference in ERK2 phosphorylation in apilimod-treated cells compared to control cells (Figure 3.1.6A, lane 3, $4 \& 5, \mathrm{~B})$. This suggests that ERK2 remains active during apilimod treatment and is not responsible for TFEB nuclear localization.

We also evaluated the contributions of GSK3$\beta$. GSK3 $\beta$ was suggested to phosphorylate TFEB independently of mTORC1 at Ser134 and Ser138 (L. Li et al., 2018; Y. Li et al., 2016). GSK3 comes in two isoforms, GSK3 $\alpha$ and GSK3 $\beta$. Activity of GSK is inversely correlated to phosphorylation at Ser21 and Ser9, respectively (Sutherland \& Cohen, 1994; Sutherland, Leighton, \& Cohen, 1993). Phosphorylation at these residues creates a pseudo-substrate that competitively binds to the active site of GSK3, blocking entry of other substrates, resulting in autoinhibition (Frame, Cohen, \& Biondi, 2001). Therefore, increased phosphorylation of GSK $3 \alpha / \beta$ is related to decreased activity. Compared to control cells, apilimod-treatment appeared to increase phosphorylation of both GSK3 isoforms (Figure 3.1.7). After 1 h, GSK3 $\alpha$ phosphorylation increased $1.7 \pm 0.4$ times relative to control (Figure 3.1.7A, lane 5), while 
GSK3 $\beta$ phosphorylation increased $2.2 \pm 0.4$ fold relative to control (Figure 3.1.7B, lane 5). For comparison, we used torin1 as a positive control for GSK3 inhibition, as S6K can phosphorylate GSK3 (Cross, Alessi, Cohen, Andjelkovich, \& Hemmings, 1995). Torin1-treated cells reduced GSK3 $\alpha$ and GSK3 $\beta$ phosphorylation to $0.61 \pm 0.02$ fold (Figure 3.1.7A, lane 2) and $0.58 \pm 0.09$ fold of control (Figure 3.1.7B, lane 2). To further investigate the role of GSK3 on TFEB nuclear localization, we transfected our cells with constitutively active GSK3 $\beta$ where S9 is mutated to alanine. Loss of this phospho-site keeps the active site of GSK3 $\beta$ free and uninhibited. However, co-expression of GSK33-S9A with TFEB-GFP did not reduce TFEB nuclear localization significantly compared to wild-type GSK3 $\beta$ during PIKfyve inhibition (Figure A1.1). However, this result is not conclusive. GSK3 signaling is complex with many factors influencing activity, including localization, phosphorylation, redundancy between isoforms, and expression levels of the mutant relative to endogenous (Bechard \& Dalton, 2009; Cole, Frame, \& Cohen, 2004; Patel $\&$ Woodgett, 2017). Thus, further work needs to be done to validate the contribution of GSK3 in this pathway. While GSK3 may not alter TFEB activation in PIKfyve-inhibited cells, the relationship between GSK3 and PIKfyve may be worth exploring as a previously unreported connection.

\subsubsection{Acute PIKfyve inhibition does not increase lysosomal protein expression}

PIKfyve inhibition leads to lysosome enlargement, that may require de novo lysosome biogenesis. TFEB is known as a master regulator of lysosome biogenesis by increasing expression of CLEAR network genes (Sardiello et al., 2009). To evaluate changes in gene expression in our system, we specifically monitored the levels of the aspartyl protease cathepsin D, the membrane protein LAMP1, the calcium channel MCOLN1, and two subunits of the V- 
ATPase, ATP6V1H and ATP6V1D. After $3 \mathrm{~h}$ of PIKfyve inhibition, the mRNA levels of all five genes were significantly increased by 33-98\% over control (Figure 3.1.8A, apilimod-treated). These changes to transcription compared favourably to the changes in gene expression during mTOR inhibition in the same time frame (Figure 3.1.8A, torin1-treated). To further confirm de novo lysosome biosynthesis in PIKfyve-inhibited cells, we checked the protein abundance of the corresponding genes. Surprisingly, protein levels of cathepsin D and V-ATPase V1H did not increase with 3 or $6 \mathrm{~h}$ of PIKfyve inhibition (Figure 3.8B, C). LAMP1 showed a slight, but significant increase of $27 \pm 6 \%$ after $6 \mathrm{~h}$ of PIKfyve inhibition, many hours after TFEB nuclear localization and lysosome enlargement. This increase in LAMP1 may be consistent with increases in LAMP2, another lysosome membrane protein, observed by Ferguson et al., possibly due to autophagosome build up during PIKfyve inhibition (Cole J. Ferguson et al., 2009). These results may suggest lysosome biogenesis does not contribute to acute lysosome enlargement.

3.1.5 Biosynthesis is not required for acute lysosome enlargement in PIKfyve inhibited cells.

Since lysosomal protein abundance does not appear to increase until many hours after TFEB nuclear localization, we aimed to understand whether TFEB was dispensable for acute lysosomal enlargement. If TFEB contributed to lysosome enlargement, lysosomes during PIKfyve inhibition of TFEB knockout cells may be smaller compared to wild-type cells. To do this, we obtained RAW cells knocked out for $t f e b^{-1-}, t f e 3^{--}$, or both from the Puertollano lab (Pastore et al., 2016) to evaluate the number and size of lysosomes during PIKfyve inhibition. First, we manually analyzed vacuole diameter using DIC optics by arbitrarily defining vacuoles has having a diameter greater than $1.5 \mu \mathrm{m}$. Using this metric, we counted the number and measured the diameter of vacuoles present in $t f e b^{-/}$and/or $t f e 3^{-/-}$cells after $1 \mathrm{~h}$. Consistent with 
the lack of protein increase, $t f e b^{-/-}$and/or $t f e 3^{-/-}$RAW cells did not have significantly fewer (Figure 3.1.9A, bottom left) or smaller (Figure 3.1.9B, bottom right) vacuoles compared to wildtype RAW cells $(3.6 \pm 0.5$ vacuoles with average diameter of $2.9 \pm 0.5 \mu \mathrm{m})$ when treated with apilimod.

We followed up these results by performing more robust, volumetric analysis of prelabeled lysosomes to determine the number and volume of each lysosome. In collaboration with Dr. Matthew Gray, we found that $t f e b^{-/-}$and/or $t f e 3^{-/-}$cells did not have significantly fewer lysosomes or significantly less lysosomes volume compared to wild-type RAW cells during apilimod-treatment (Figure 3.1.9B). These results suggest that TFEB and/or TFE3 are not required for acute lysosomal enlargement in apilimod-treated cells. Since MiTF can also contribute to lysosome biogenesis, we ruled out redundancy by analyzing the lysosome volume in HeLa cells knocked out for $t f e b^{-/-}, t f e 3^{-/-}$and $m i t f^{/-}$by CRISPR/Cas9, gifted to us by the Youle lab (Nezich et al., 2015). In collaboration with Golam Saffi, he showed that triple knockout cells were not significantly different with respect to lysosome number, volume per lysosome, or total volume per cell compared to wild-type HeLa cells (Figure A1.2) (Choy et al., 2018). Consistent with RAW cells, the number and size of lysosomes during PIKfyve-inhibition do not change in the presence or absence of MiT/TFE family members in HeLa cells.

To further confirm that protein synthesis is not required for acute lysosome enlargement during PIKfyve inhibition, we blocked translation using cycloheximide. Cycloheximide is an antibiotic produced by Streptomyces griseus that blocks protein elongation by binding to the Esite of the 60S ribosome (Schneider-Poetsch et al., 2010). Consistent with our previous results, cells treated with cycloheximide did not reduce the average number or volume of lysosomes (Figure 3.1.10A). Cells treated with apilimod and cycloheximide had $29 \pm 13$ lysosomes with an 
average volume of $8.1 \pm 2.1 \mu^{3}$, while cells treated with apilimod alone had $30 \pm 9$ lysosomes having $8.3 \pm 2.5 \mu \mathrm{m}^{3}$ average volume. Efficacy of cycloheximide was evaluated by puromycin incorporation assay and by checking the abundance of fast turnover protein p53. Puromycin blocks protein translation by prematurely terminating peptide elongation after its incorporation into growing peptides (Yarmolinsky \& Haba, 1959). In control cells, puromycin labelled proteins that were actively translated, while cycloheximide largely blocked the production of puromycylated proteins (Figure 3.1.10B, lane $3 \& 4$ ). Similarly, p53 is a fast turnover protein with a half-life less than 20 minutes (Giaccia \& Kastan, 1998). Blocking translation with cycloheximide blocks synthesis of new p53 while allowing turnover of existing p53. In accordance, p53 abundance was significantly reduced to $19.6 \pm 5.1 \%$ in cycloheximide-treated cells compared to the vehicle-treated control. Together, these results suggest that protein synthesis is not required for lysosome enlargement during PIKfyve inhibition.

Although de novo biosynthesis may not be required for acute lysosome enlargement, we noticed that in all cases, the number of individual lysosomes decreased while the volume of individual lysosomes increased during PIKfyve inhibition. We hypothesized that there may be an imbalance in the relative fusion-fission rates of lysosomes, shifting towards increased rates of lysosome fusion during PIKfyve inhibited. Therefore, blocking lysosome fusion during PIKfyve inhibition may prevent lysosome enlargement and reduction in lysosome number. By blocking components of lysosome fusion during PIKfyve inhibition, lysosomes became smaller and cells resisted lysosome enlargement and retained higher lysosome number compared to apilimod-only treated cells. This work was also featured in our journal publication by my co-first author, Golam Saffi (Choy et al., 2018). Overall, despite TFEB activation during PIKfyve inhibition, TFEB is not required for lysosome enlargement. Furthermore, de novo lysosome biosynthesis appears to 
be dispensable for acute lysosome enlargement during PIKfyve inhibition. Further work should be done to understand the functional consequence of TFEB activation during PIKfyve inhibition. 


\subsection{Phagocytosis induces TFEB nuclear localization and enhances proteolysis}

\section{$\underline{\text { 3.2.1 Fc } \gamma \text { receptor engagement leads to TFEB nuclear localization }}$}

Overexpression of TFEB enhances degradation of lysosomal cargo (Settembre et al., 2011). Similarly, phagocytosis of microbes by phagocytes sequesters the potential pathogens in a membrane-bound phagosome. These phagosomes are subsequently degraded by the lysosome to neutralize the potential pathogens (Wong et al., 2017). We wondered if phagocytic cells activated TFEB-dependent lysosome biogenesis to enhance degradation of their phagosomes. To

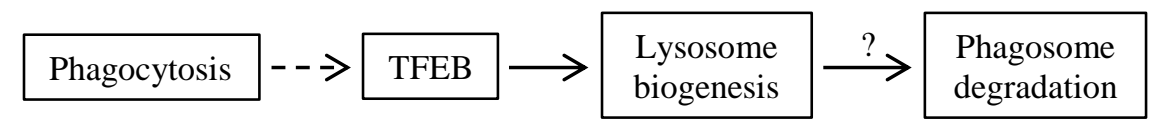

test whether TFEB was activated by phagocytosis, we challenged RAW macrophages with IgGopsonized beads. After 30 min of internalization, $67 \pm 6 \%$ of cells had nuclear TFEB-GFP after a 60 min chase period compared to $7 \pm 2 \%$ resting cells (Figure 3.2.1A). Likewise, the nuclear-tocytosolic ratio of endogenous TFEB increased to $1.3 \pm 0.6$ after 60 min of phagocytosis from 0.3 \pm 0.1 in control cells (Figure 3.2.1B). We wondered if $\mathrm{Fc} \gamma$ receptor engagement was sufficient to induce nuclear localization of TFEB. Therefore, we challenged our cells with heat-aggregated IgG, multivalent forms of IgG that are internalized by endocytosis (Tse et al., 2003). Endocytosis via the Fc $\gamma$ receptor led to increased nuclear localization of cells expressing TFEB-GFP in as little as 10 minutes $(56 \pm 26 \%)$ compared to resting cells $(18 \pm 9 \%)$, with TFEB-GFP nuclear localization plateauing after $60 \min (97 \pm 2 \%)$ (Figure 3.2.1C).

We also investigate whether TFEB nuclear localization could be generalized to the immune response. To evaluate the role of other immune stimuli, we challenged our cells with cytokines. These are chemical messages secreted by immune cells that can polarize macrophages (Martinez \& Gordon, 2014). We use interferon $\gamma$ (IFN $\gamma$ ) and IL-12 as pro-inflammatory 
cytokines, and IL-4 and IL-10 as anti-inflammatory cytokines. In all cases, TFEB-GFP did not increase in its nuclear localization even after $4 \mathrm{~h}$ of stimulation compared to the control condition $(24 \pm 4 \%$ ) (Figure 3.2.2). Taken together, TFEB nuclear localization may specifically respond to specific immunological stimuli, rather than all immune signals.

\subsubsection{Lysosomes become more proteolytic after TFEB-induced lysosome biogenesis}

Lysosome gene transcription coincides with TFEB nuclear localization (Sardiello et al., 2009). To check if lysosome gene expression increased with IgG-opsonized beads, we checked the mRNA levels of the protease CTSD, subunit of the V-ATPase ATP6V1H, membrane protein LAMP1, and calcium channel MCOLN1. In collaboration with Dr. Matthew Gray, we showed that only some genes were upregulated with phagocytosis (Figure 3.2.3A). Specifically, ATP6V1H and CTSD increased $>50 \%$ relative to control after $4 \mathrm{~h}$ of phagocytosis. In contrast, there was no significant increase in LAMP1 or MCOLN1 (Figure 3.2.3A). For comparison, RAW cells treated with torin 1 for the same time frame significantly increased mRNA of all genes as seen previously (Figure 3.2.3A, Figure 3.1.8A). Furthermore, work done by Dr. Gray showed TFEB activation by heat-aggregated IgG did not increase lysosome number (Figure A1.3) (Gray et al., 2016). Together, the selective increase in ATP6V1H and CTSD may make existing lysosome more acidic and more proteolytic, rather than make more lysosomes.

TFEB-mediated lysosome biogenesis was previously shown to enhance degradation of cargo (Sardiello et al., 2009). To test the hypothesis that lysosomes become more proteolytic, we track the fluorescence of dye-quenched bovine serum albumin (DQ-BSA). This BSA molecule is heavily labeled with a BODIPY fluorescent dye such that the emission fluorescence is quenched. As the molecule is degraded by the lysosomes, the BODIPY is liberated and increases 
fluorescence. In this way, increased fluorescence from DQ-BSA suggests increased proteolysis by the lysosome. We pre-activate TFEB by challenging RAW macrophages with aggregated $\operatorname{IgG}$ for 2 for $6 \mathrm{~h}$ and allow TFEB activation to upregulate lysosome biogenesis. After the incubation period, DQ-BSA was endocytosed and the fluorescence emitted was observed by flow cytometry. To control for rates of endocytosis, cells co-endocytosed a fluorescent dextran. In collaboration with Monica Dayam, we showed that pre-activation of TFEB enhanced fluorescence of DQ-BSA relative to the control cells in a time dependent manner. Compared to control, $2 \mathrm{~h}$ pre-treatment with aggregated IgG increased DQ-BSA fluorescence by $34 \pm 16 \%$ while $6 \mathrm{~h}$ pre-treatment enhanced DQ-BSA fluorescence by $68 \pm 32 \%$ (Figure 3.2.3B). Together, these results suggest that $\mathrm{Fc} \gamma$ receptor activation leads to more degradative lysosomes.

\subsubsection{Phagocytosis leads to TFEB dephosphorylation}

TFEB becomes active in response to autophagy to make more lysosomes for autophagosomes degradation (Settembre et al., 2011). Specifically, TFEB activation is dependent on loss of phosphorylation by mTORC1 inactivation and calcineurin activation (Medina et al., 2015; Roczniak-Ferguson et al., 2012). To understand how the Fc $\gamma$ receptor activates TFEB to enhance degradation of phagosomes, we first checked the phosphorylation status of TFEB using a combination of standard SDS-PAGE gels and Phos-tag gels, with total TFEB antibody or phospho-S142 TFEB antibody (Figure 3.2.4). In addition to IgG beads, we also used E. coli as a more physiological model. In standard SDS-PAGE gels (Figure 3.2.4A,C), total TFEB mobility increased when RAW cells were challenged with phagocytic targets (Figure 3.2.4A). This change in mobility is comparable to mTOR inhibition with torin1 (Figure 3.2.4A, lane 4). Using Phos-tag gels, we also saw a redistribution of various slower-moving TFEB bands in the control 
condition to faster running bands in cells that phagocytosed particles (Figure 3.2.4C). We compared these Western blots against total TFEB to blots probed with anti-pS142 antibodies (Figure 3.2.4B,D). TFEB is thought to be phosphorylated by mTORC1 and/or ERK2 at S142, modulating nuclear localization (Puertollano et al., 2018). With SDS-PAGE gels, the pTFEB antibody strongly detects a slow running band in control cells (Figure 3.2.4B, indicated with **). Phagocytosis lead to the enrichment of a faster running band (Figure 3.2.4B, indicated with *). This same pattern appears in Phos-tag gels stained for pTFEB (Figure 3.2.4D). Together, these results suggest phagocytosis induces TFEB dephosphorylation, producing banding patterns distinct from control cells. Despite the many possible phosphorylation sites, TFEB appears to retain the phosphorylation of S142 during phagocytosis. Further investigation will be required to understand the specific bands detected by the pS142 antibody and how these forms of TFEB are phosphorylated.

To examine the role of mTORC1 inactivation on TFEB dephosphorylation, we checked the phosphorylation status of S6K. Again, S6K phosphorylation at T389 is proportional to mTORC1 activity. RAW cells challenged with IgG-opsonized beads or E. coli did not decrease S6K phosphorylation (Figure 3.2.5). Together, these results suggest TFEB activation is dependent on dephosphorylation despite consistent activation of mTORC1 during phagocytosis.

\subsubsection{Fc $\gamma$ receptor signaling leading to actin polymerization is required for TFEB nuclear} localization

The signaling pathway downstream of the Fc $\gamma$ receptor is complex, activating many signaling branches that culminate in the polymerization of actin and membrane remodelling to enact particle internalization (Flannagan, Jaumouillé, \& Grinstein, 2012). Since mTORC1 
remains active during phagocytosis, we hypothesized that some aspect of Fc $\gamma$ receptor may regulate TFEB activation. To understand the signaling requirements for TFEB nuclear localization, we targeted key junctions in Fcy receptor signaling (Figure 3.2.6A). Engagement of Fc $\gamma$ receptors leads to receptor clustering and phosphorylation of the ITAM domain by Src family kinases (Ghazizadeh et al., 1994; Hamada et al., 1993; Holowka, Sil, Torigoe, \& Baird, 2007). This is the first event that leads to further downstream signaling. Blocking Src kinases with dasatinib prevented TFEB nuclear localization compared to the control cells (Figure 3.2.6B, orange). This suggests that some aspect of Fc $\gamma$ receptor signaling is required for TFEB nuclear localization.

Next, we blocked actin polymerization using cytochalasin D, which should allow signalling by engaged Fc receptors but block particle internalization. Blocking actin polymerization also inhibited TFEB nuclear localization relative to the control cells (Figure 3.2.6B, red). We also checked the contributions of ERK, which is known to stimulate the immune response by activating NF-кB and can directly act on TFEB (Garc1 \& Rosales, 2001; Settembre et al., 2011). However, blocking ERK with U0126 did not block TFEB nuclear localization (Figure 3.2.6B, U0126).

To further investigate signaling requirements for particle internalization, we targeted class I PI3K (PI3KCI) which is required for phagocytosis of large particles (Schlam et al., 2015). Interestingly, blocking PI3KCI with LY294002 led to constitutive TFEB nuclear localization (Figure 3.2.6B, blue). LY294002 is a pan-PI3K inhibitor that can also inhibit class II and III PI3K. PI3KCIII is particularly interesting because the PtdIns(3) $P$ generated by Vps34 is converted by PIKfyve into PtdIns(3,5) $P_{2}$ (Vieira et al., 2001). This further supports our results from Chapter 3.1. Therefore, we specifically targeted PI3KCI using ZSTK474. Inhibition of 
PI3KCI blocked TFEB nuclear localization (Figure 3.2.6C, blue). We further checked the requirements of phagocytosis by blocking PLC $\gamma$. PtdIns(4,5) $P_{2}$ is cleaved by PLC $\gamma$ into IP3 and DAG, the latter which is required actin independent stimulation of phagocytosis by recruiting PKC $\varepsilon$ (Botelho et al., 2000; Larsen et al., 2002).Blocking PLC $\gamma$ with pan-PLC inhibitor U73122 also blocked TFEB nuclear localization (Figure 3.2.6C, green), further supporting a requirement for particle internalization. Taken together, Fc $\gamma$ receptor signaling mediators that are necessary for particle uptake appear to be required for TFEB nuclear localization following Fcy receptor activation. Therefore, particle internalization and subsequent phagosome formation may be necessary for TFEB activation following Fc $\gamma$ receptor engagement.

3.2.5 Particle internalisation but not phagosome maturation is required for TFEB localization by the Fcy receptor

The signaling components necessary for TFEB nuclear localization suggest that the particle must be internalized to form a phagosome. To test this hypothesis, we challenged RAW

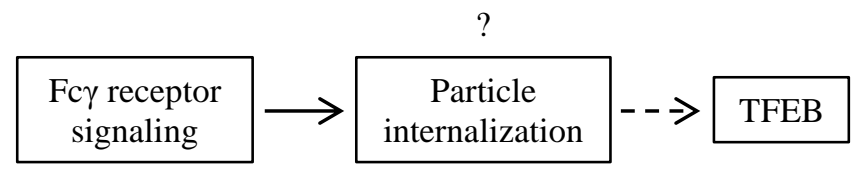

cells with oversized beads opsonized with IgG. Instead of the 3.0 or $3.87 \mu \mathrm{m}$ beads that were smaller than the cell, these oversized beads were $21.25 \mu \mathrm{m}$. Since these beads are opsonized with IgG, the RAW cells will attempt to internalize the beads but become "frustrated" and fail to internalize the particle despite downstream signaling from the receptor. Similar work done by Dr. Gray showed frustrated phagocytosis onto coverslips allowed for Fc receptor signaling, as reported by phosphorylation of Syk (Figure A1.4A) (Gray et al., 2016). Cells challenged with 3.0 $\mu \mathrm{m}$ beads induced TFEB nuclear localization $4.1 \pm 0.6$ fold over control cells (Figure 3.2.7). 
Meanwhile, cells challenged with $21.25 \mu \mathrm{m}$ beads strongly abated TFEB nuclear translocation, with only $1.8 \pm 0.6$ fold over control. This result suggests phagosomes must form to induce TFEB nuclear localization and that signalling is not sufficient to engage TFEB. These results are consistent with work from our lab that showed frustrated phagocytosis of IgG-opsonized coverslips also blocked TFEB nuclear localization (Figure A1.4B).

While it appears that the particle must be internalized to induce TFEB nuclear localization, we also wondered if phagosome-lysosome fusion is required for TFEB nuclear

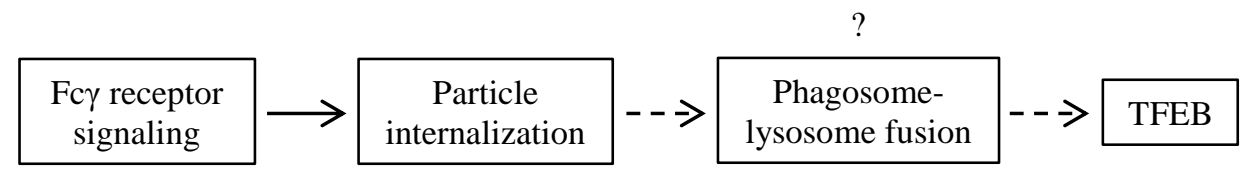

localization. Our kinetic analysis with phagocytosis of IgG opsonized beads showed TFEB-GFP localized to the nucleus between $30-60$ min post-phagocytosis (Figure 3.2.1A). This timing is consistent with the time required for the phagosome to acquire LAMP1, a marker of the late endosome and lysosome (Vieira et al., 2001). To block maturation of the phagosome, we knockdown the Rab7 GTPase. Rab7 activity is required for phagolysosome formation (Harrison et al., 2003).

Two separate sets of Rab7 knockdown were performed using different individual oligonucleotides. To confirm knockdown of Rab7, we performed Western blots following $48 \mathrm{~h}$ of siRNA gene silencing (Figure 3.2.8A). Cells knocked-down with oligonucleotide 1 or 2 showed $88 \pm 1 \%$ and $96 \pm 2 \%$ reduced Rab7 abundance relative to the non-targeting control oligo. Cells with Rab7 knockdown did not change basal TFEB localization (Figure 3.2.8B). Consistent with our hypothesis, cells knocked-down for Rab7 prevented TFEB nuclear localization with E. coli, relative to the no-knockdown condition. Specifically, the TFEB nucleus-to-cytosolic ratio was reduced to $44 \pm 10 \%$ and $44 \pm 18 \%$ of the non-targeting condition 
for the oligonucleotide 1 and 2 respectively. Further work needs to be done to confirm that maturation is impaired in Rab7 knockdown cells. Together, these results suggest that Fc $\gamma$ receptor signaling leading to particle internalization, phagosome formation, and phagosome maturation are all necessary for TFEB activation following phagocytosis. 
Chapter 3: Figures 
A<smiles>Cc1cccc(/C=N/Nc2cc(N3CCOCC3)nc(OCCc3ccccn3)n2)c1</smiles>

B
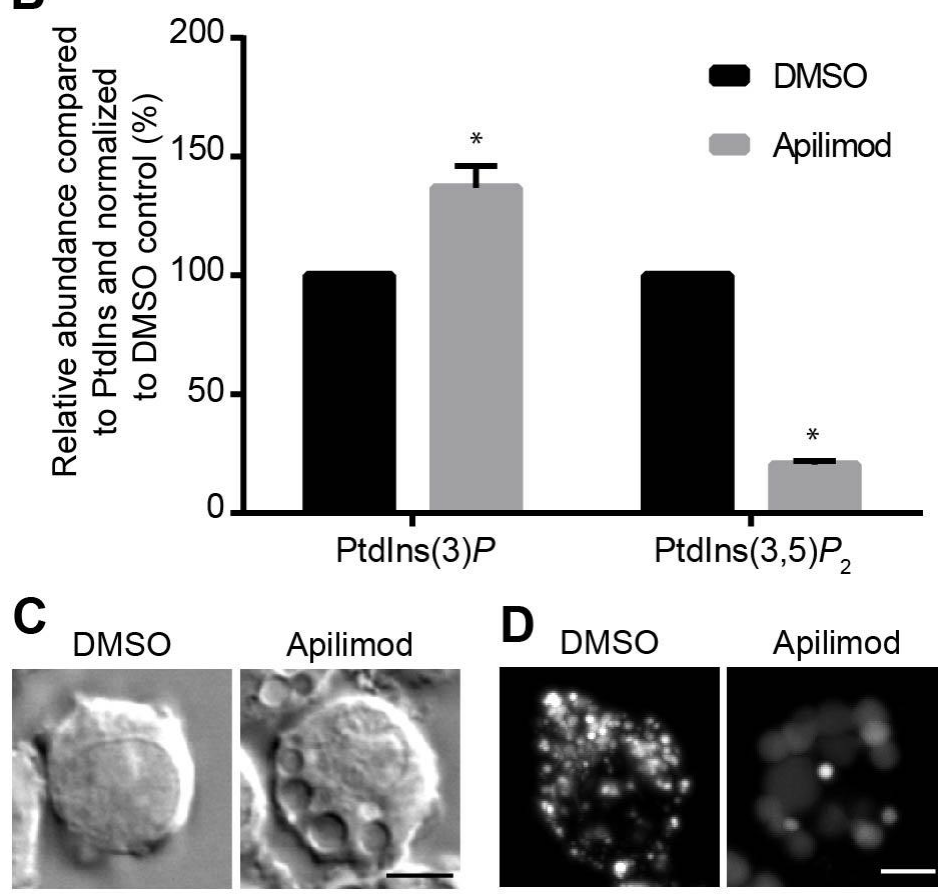

Apilimod 
Figure 3.1.1: Apilimod depletes PtdIns(3,5) $P_{2}$ in RAW macrophages leading to enlarged lysosomes. (A) Structure of apilimod is shown. (B) Quantification of $\operatorname{PtdIns(3)P~and~}$ PtdIns(3,5) $P_{2}$ in RAW cells metabolically labeled with ${ }^{3} \mathrm{H}-m y o$-inositol and HPLC-coupled flow scintillation. Cells were treated with $20 \mathrm{nM}$ apilimod for $1 \mathrm{~h}$ and compared against the DMSOtreated control. Data shown is the mean abundance \pm SEM by measuring the amount of each PtdInsP, normalized against the parental PtdIns peak before comparison against the vehicletreated control. Asterisks (*) indicate statistical significance compared to the DMSO-treated control condition ( $(<0.05)$ using Student's t-test (Ho et al., 2015). (C, D) Apilimod induces lysosomal enlargement in RAW macrophages. (C) Lysosomes become clearly visible by differential interference contrast microscopy after $1 \mathrm{~h}$ of $20 \mathrm{nM}$ apilimod treatment relative to the vehicle-treated control. (D) Lysosomes can be visualized by fluorescence microscopy when prelabeled with a fluid-phase marker like lucifer yellow. In vehicle-treated cells, lysosomes are small and numerous, but become greatly enlarged and fewer in number after treatment with 20 $\mathrm{nM}$ apilimod for $1 \mathrm{~h}$ compared to the vehicle-treated control condition. Scale bars indicate $5 \mu \mathrm{m}$. 

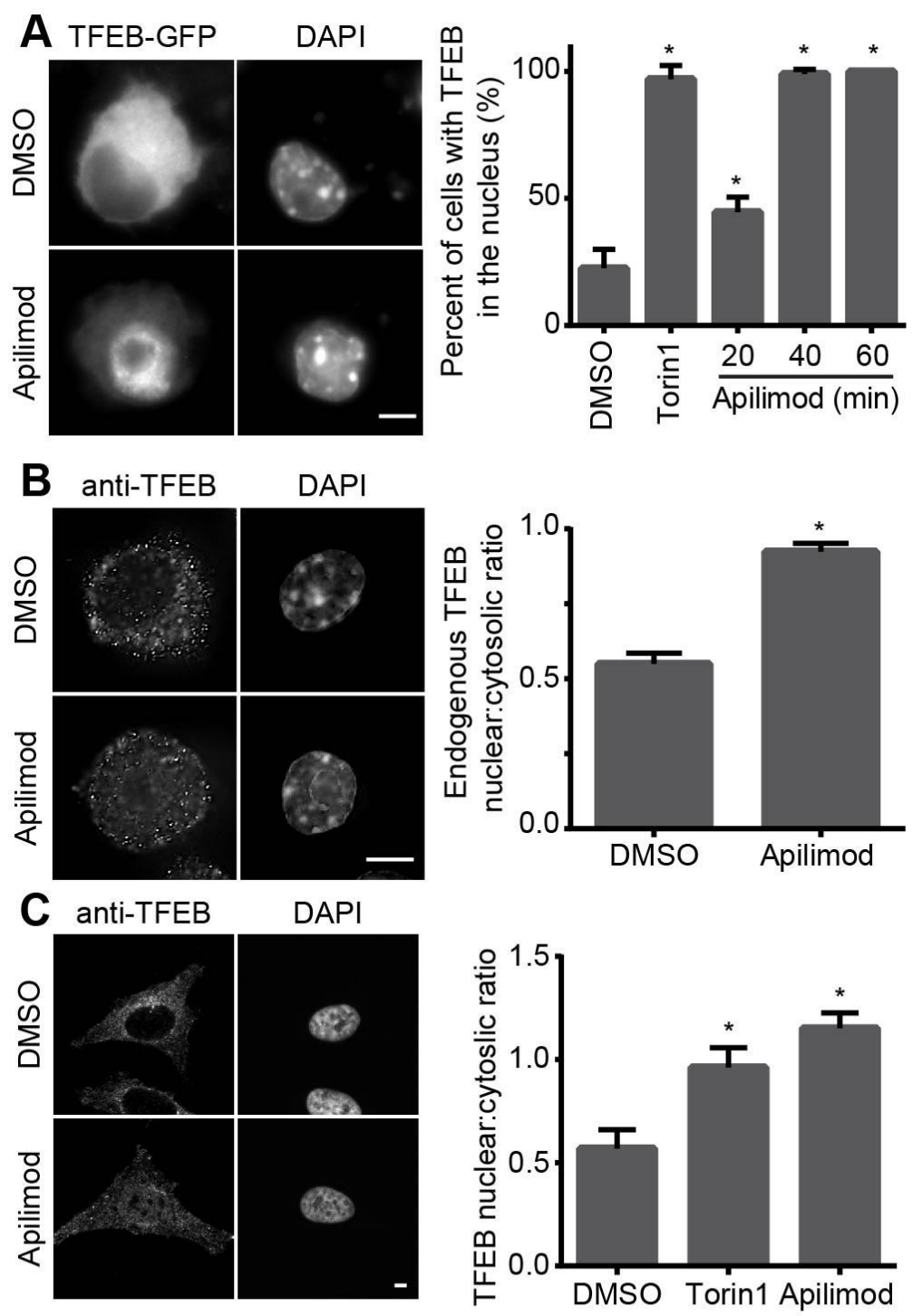
Figure 3.1.2: PIKfyve inhibition with apilimod induces TFEB nuclear localization. (A, B) RAW cells expressing TFEB-GFP (A) or stained for endogenous TFEB (B) treated with DMSO or 20 nM apilimod for $1 \mathrm{~h}$. (C) HeLa cells stained for endogenous TFEB treated with vehicle or 200 $\mathrm{nM}$ apilimod for $1 \mathrm{~h}$. Where indicated, the nucleus is counter-stained with DAPI. Nuclear translocation of TFEB was expressed as a percent of cells with greater average nuclear fluorescence compared to the cytosol of TFEB-GFP (A) or the nuclear:cytosolic ratio of their respective mean fluorescence intensity for endogenous TFEB $(B, C)$. Quantifications shown are the mean \pm SEM for at least three independent experiments with a minimum of 30 cells counted per condition per experiment. Torin1 is used as a positive control through inhibition of mTOR and applied for $1 \mathrm{~h}$ at $100 \mathrm{nM}$. Asterisks (*) indicate statistical difference compared to the vehicle treated control $(\mathrm{p}<0.05)$ using one-way ANOVA with Tukey's post-hoc test $(\mathrm{A}, \mathrm{C})$ or Student's t-test (B). Scale bars indicate $5 \mu \mathrm{m}$. 

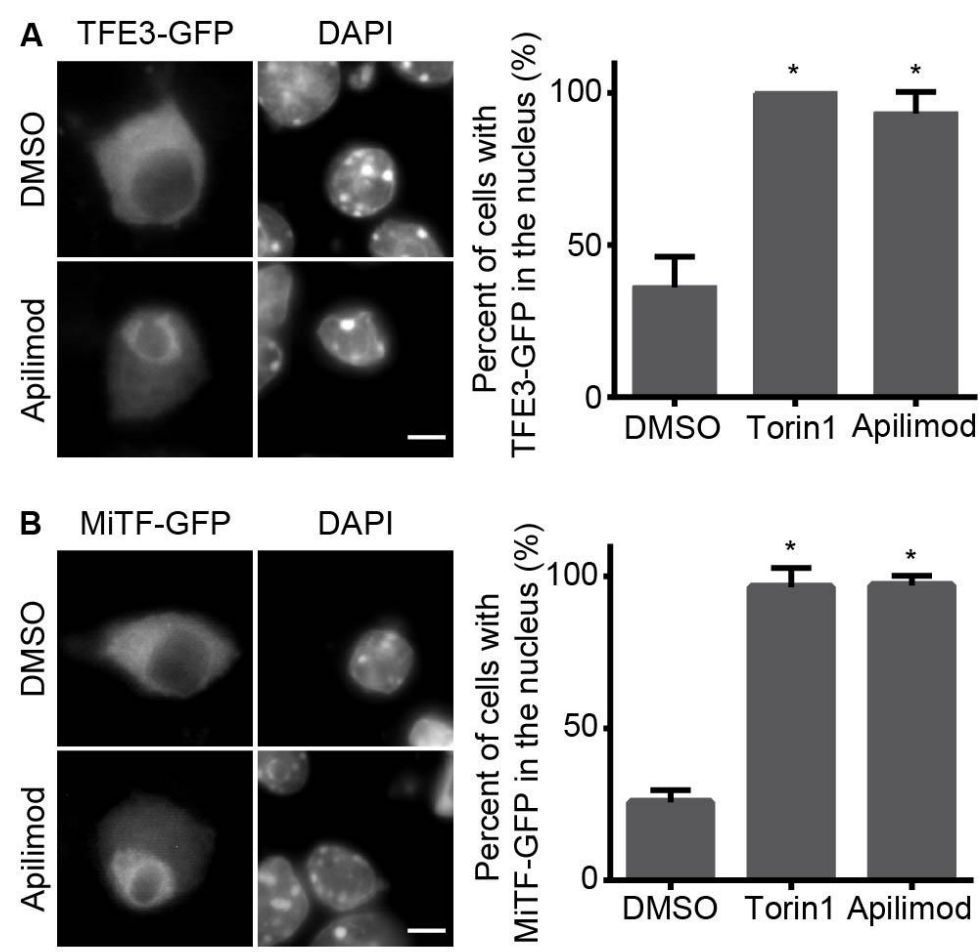
Figure 3.1.3: PIKfyve inhibition also induces nuclear localization of other MiT/TFE family transcription factors. RAW cells transfected with either TFE3-GFP (A) or MiTF-GFP (B) treated with $20 \mathrm{nM}$ apilimod for $1 \mathrm{~h}$ or DMSO control. The accompanying quantification shows the percent of cells with greater average fluorescence intensity in the nuclear compared to the cytosol. Where indicated, DAPI is used to stain the nucleus. The accompanying quantifications show the mean \pm SEM for at least three independent experiments with a minimum of 30 cells counted per condition per experiment. Inhibition of mTOR with $100 \mathrm{nM}$ torin 1 for $1 \mathrm{~h}$ is used as a positive control. Asterisks (*) indicate statistical difference compared to the vehicle-treated control $(\mathrm{p}<0.05)$ using one-way ANOVA with Turkey's post-hoc test. Scale bars indicate $5 \mu \mathrm{m}$. 


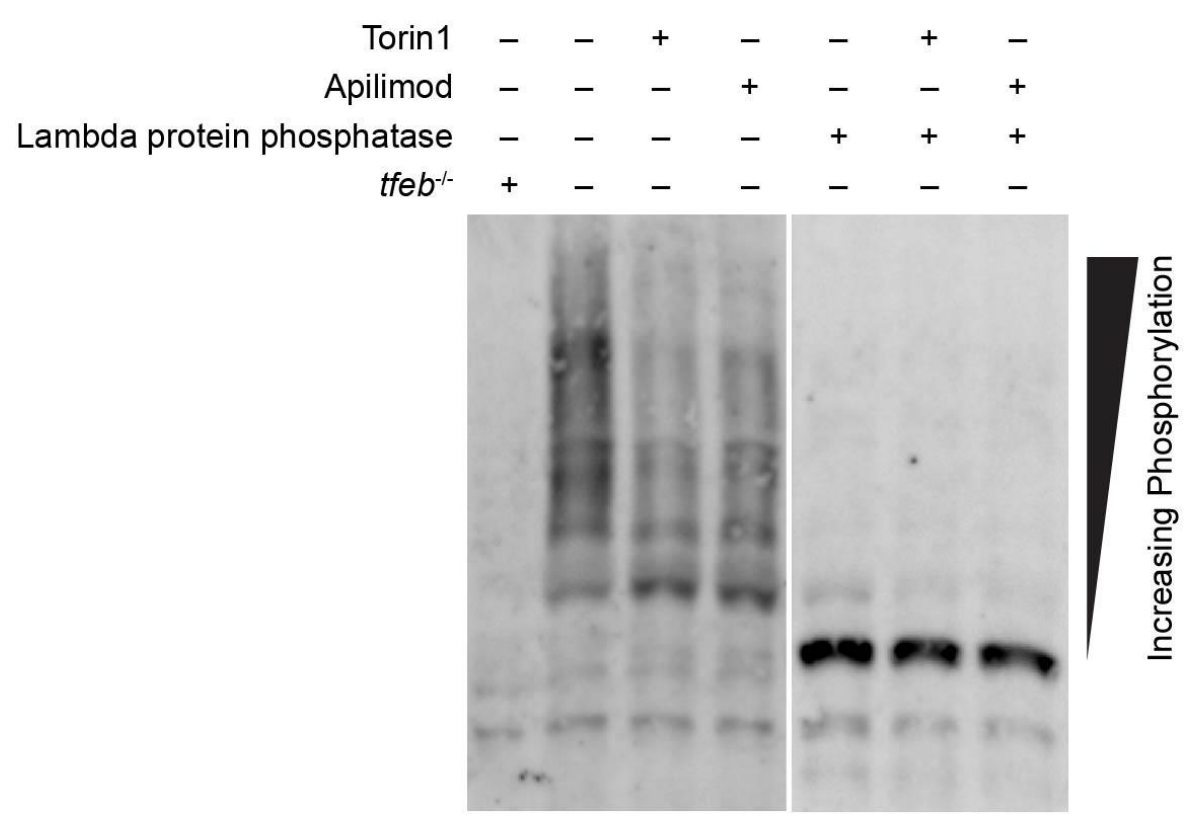


Figure 3.1.4: Apilimod treatment changes TFEB phosphorylation pattern. Western blot of whole cell lysates run on SDS-PAGE gels supplemented with Phos-tag acrylamide and $\mathrm{Mn}^{2+}$. A representative blot from three independent experiments is shown. TFEB gel mobility of DMSOtreated control cells is compared against $100 \mathrm{nM}$ torin1- and $20 \mathrm{nM}$ apilimod-treated cells for 1 h. Bands appearing higher on the blot have greater phosphorylation. Specificity of anti-TFEB antibody is confirmed with the absence of bands in $t f e b^{-/}$RAW whole cell lysates. For comparison, most bands seen in wild-type RAW lysates collapse into a single fast running band after dephosphorylation with lambda protein phosphatase. 


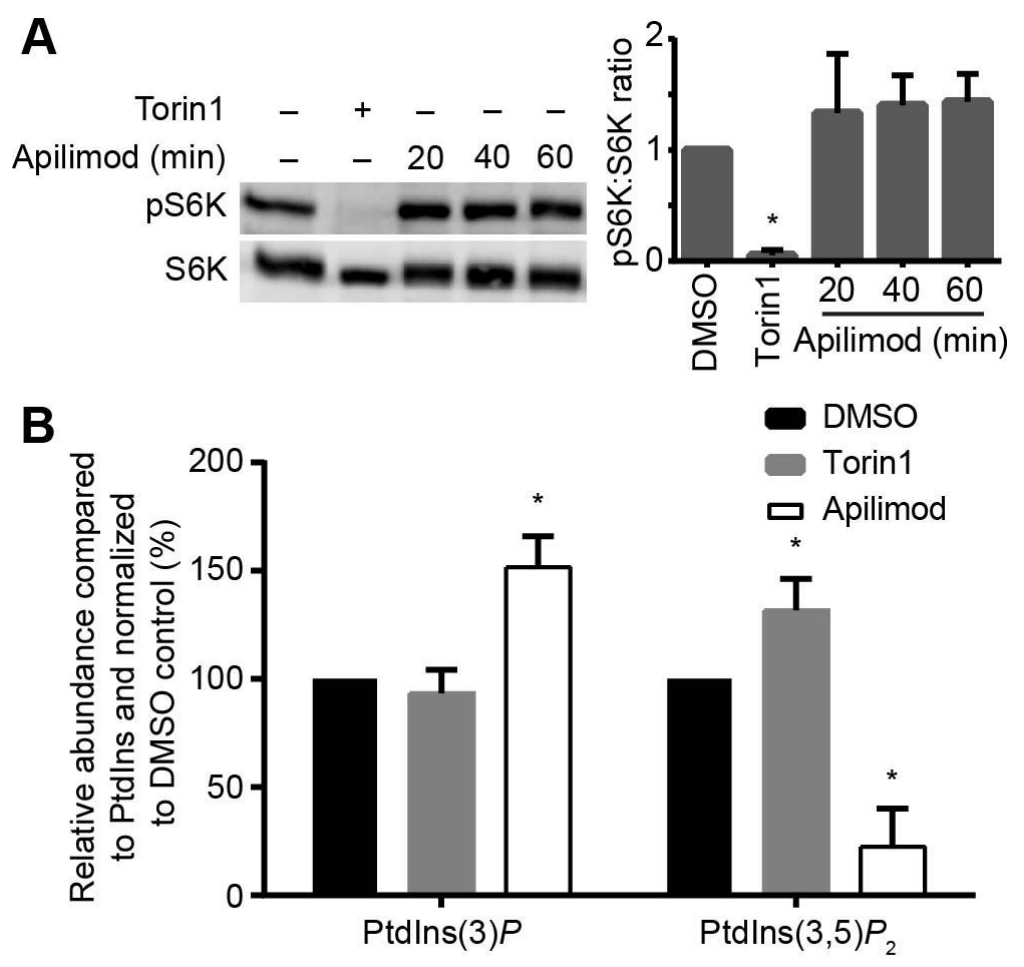


Figure 3.1.5: PIKfyve and mTORC1 function independently. (A) mTORC1 activity based on phosphorylation of p70-S6K. Cells were treated with either vehicle, $20 \mathrm{nM}$ apilimod for the times indicated, or $100 \mathrm{nM}$ torin 1 for $1 \mathrm{~h}$ as a positive control. A representative blot is shown from four independent experiments. The accompanying quantification shows the mean abundance \pm SEM of phospho-p70-S6K at T389 compared to total p70-S6K and normalized against the vehicle-treated control. (B) PtdIns(3,5) $P_{2}$ levels during mTORC1 inhibition. PtdInsP were metabolically labelled with ${ }^{3} \mathrm{H}-m y o$-insotiol and subjected to HPLC-coupled flow scintillation. Cells were treated with either vehicle, $100 \mathrm{nM}$ torin1 or $20 \mathrm{nM}$ apilimod for $1 \mathrm{~h}$. Shown is the quantification of the mean abundance \pm SEM of each PtdInsP normalized against the parental PtdIns and compared against the vehicle-treated control condition. Asterisks (*) indicate statistical difference compared to the vehicle treated control $(\mathrm{p}<0.05)$ using multiple Student's t-test with Bonferroni correction. 


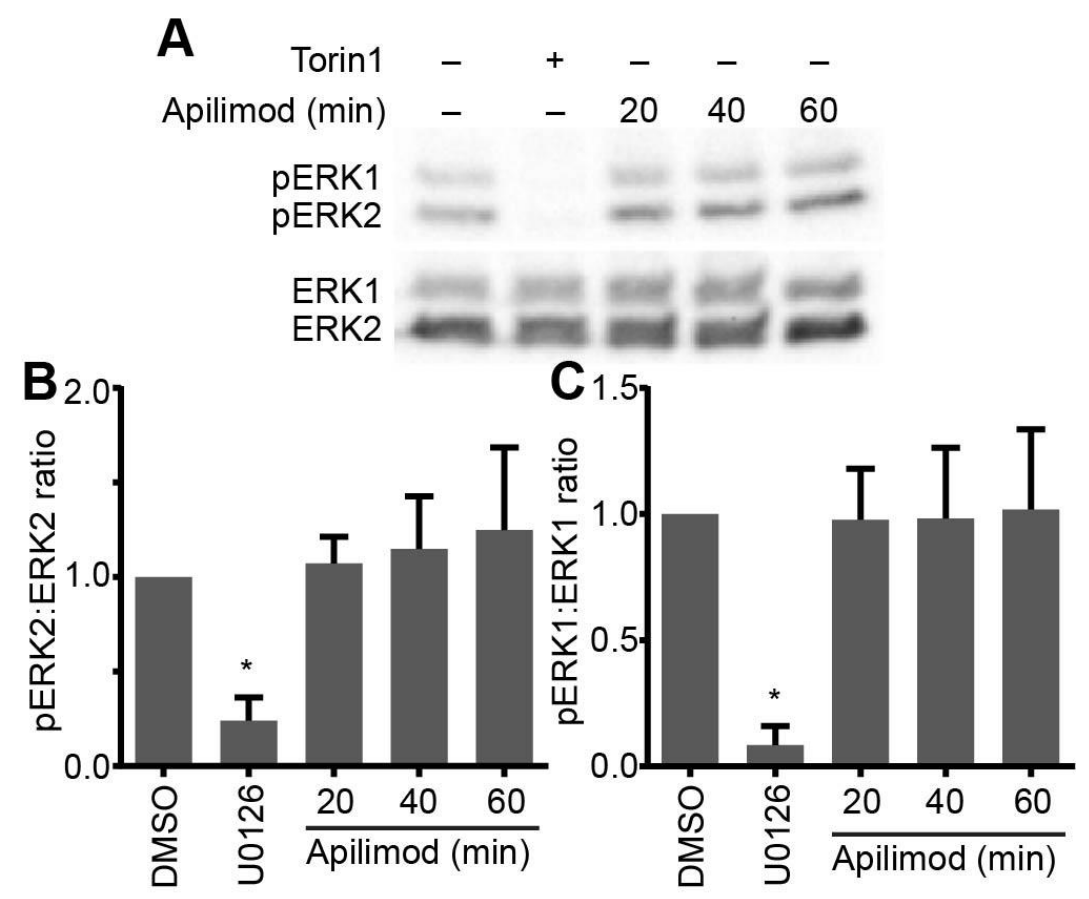


Figure 3.1.6: ERK2 remains active during PIKfyve inhibition. Western blot analysis of ERK1/2 phosphorylation. Cells were treated with either vehicle, $20 \mathrm{nM}$ apilimod for the times shown, or $10 \mu \mathrm{M}$ ERK inhibitor U0126 for $1 \mathrm{~h}$ as a positive control. (A) Shown is a representative blot of three independent experiments. (B,C) The accompanying quantification shows the mean abundance \pm SEM of (B) phospho-ERK2 at T185/Y187 compared to total ERK2 and normalized against the vehicle-treated control or (C) phospho-ERK1 at T202/Y204 compared to total ERK1 and normalized against the vehicle-treated control. Asterisks (*) indicate statistical difference compared to the vehicle treated control $(\mathrm{p}<0.05)$ using multiple Student's t-test with Bonferroni correction. Unpublished data. 

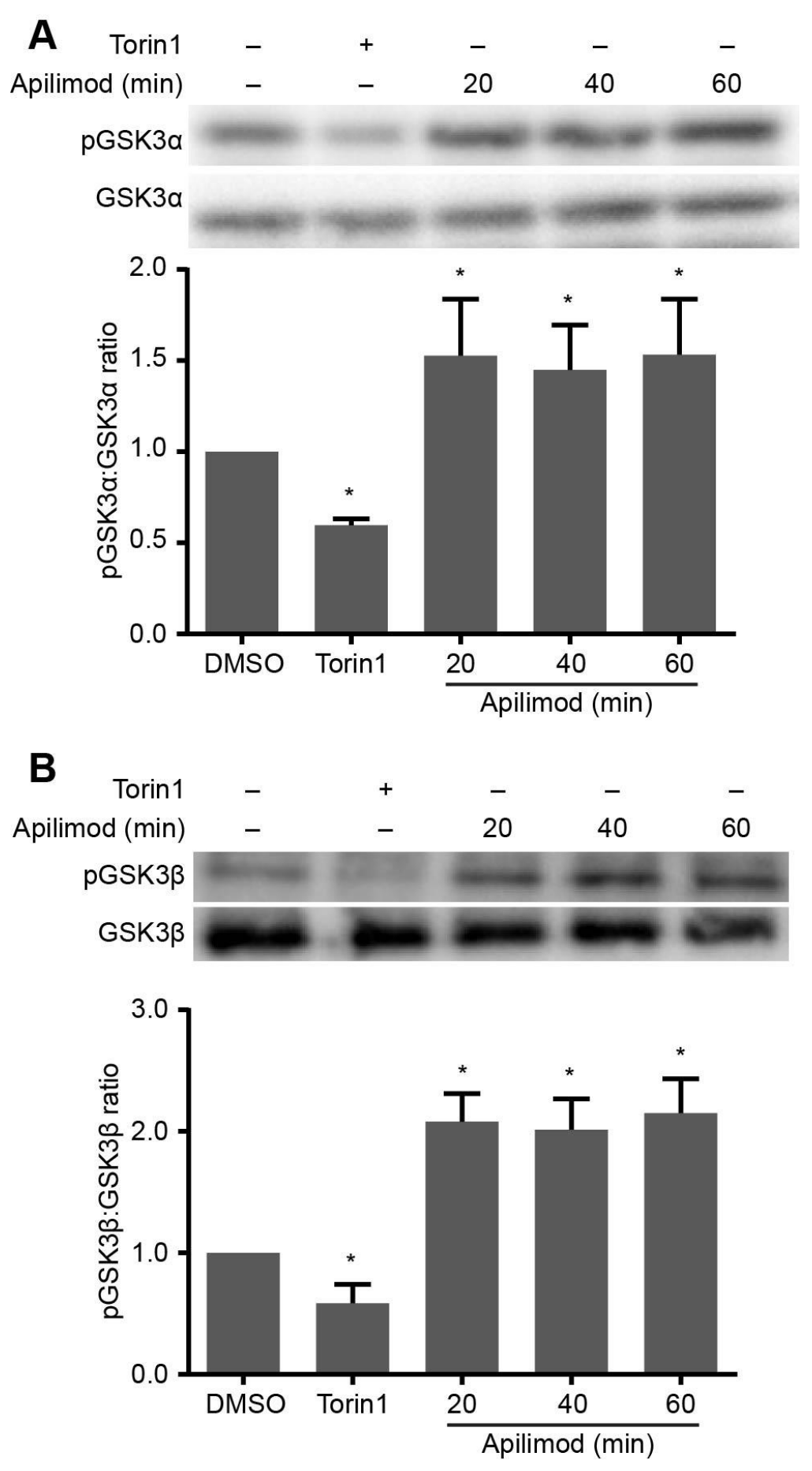
Figure 3.1.7: PIKfyve inhibition increases inhibition of GSK3 $\alpha / \beta$. Western blot analysis of GSK3 $\alpha / \beta$ phosphorylation. Cells were treated with vehicle, $20 \mathrm{nM}$ apilimod for the times indicated, or $100 \mathrm{nM}$ torin 1 for $1 \mathrm{~h}$ as a positive control. A representative image is shown of three independent experiments. The accompanying quantification shows the mean abundance \pm SEM of phospho-GSK3 $\alpha / \beta$ at S21/9 respectively compared to total GSK $3 \alpha / \beta$ respectively and normalized against the vehicle-treated control. Asterisks $(*)$ indicate statistical difference compared to the vehicle treated control $(\mathrm{p}<0.05)$ using multiple Student's t-test with Bonferroni correction. Unpublished data. 


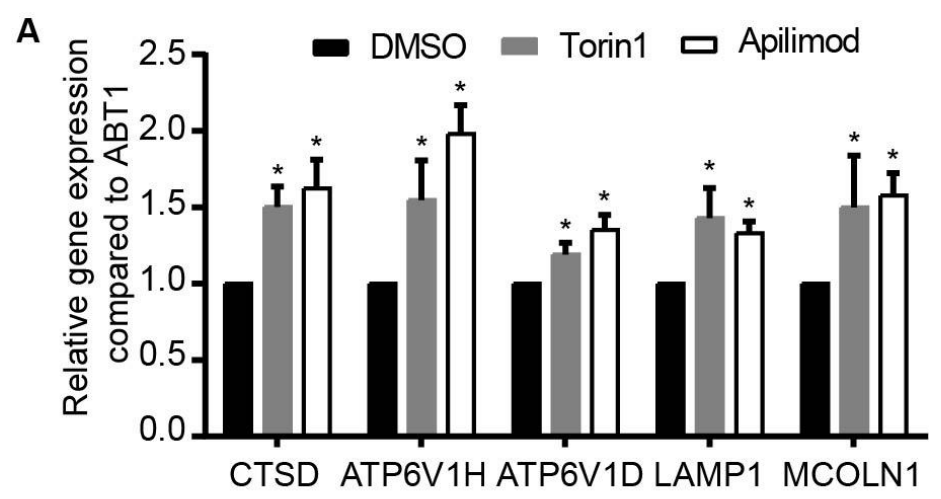

B
Torin 1
Apilimod
$6 \mathrm{~h}$
-
$3 \mathrm{~h}$
$6 \mathrm{~h}$
LAMP1

vATPase V1H

55

Cathepsin D

28

HSP60

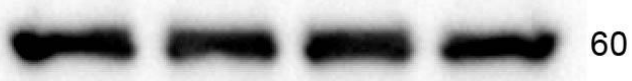

C

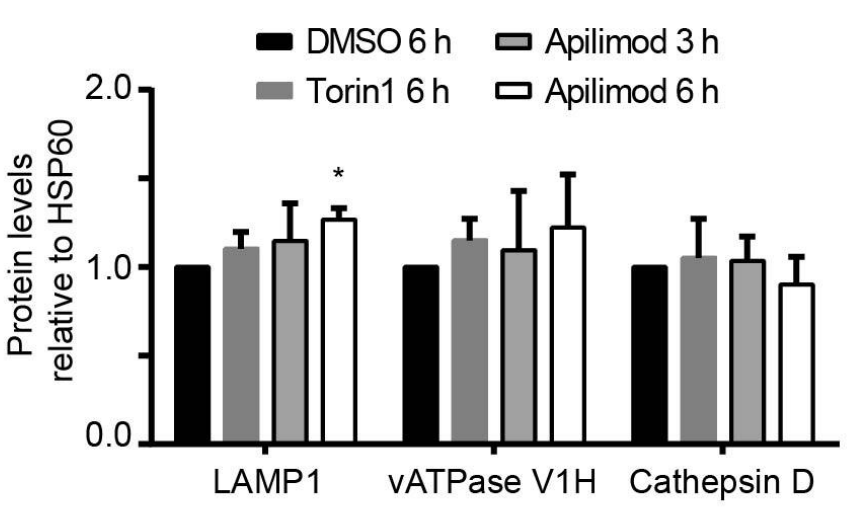


Figure 3.1.8: PIKfyve inhibition increases lysosome gene transcription but not translation. (A) Quantification of select lysosomal gene mRNA by qRT-PCR. Total RNA was extracted from cells treated with vehicle, $100 \mathrm{nM}$ torin 1 or $20 \mathrm{nM}$ apilimod for $3 \mathrm{~h}$. Shown is the mean mRNA levels \pm SEM relative to the control gene ABT1 and normalized against the vehicle-treated control condition from seven independent experiments. (B) Western blot of select lysosomal proteins. Whole cell lysates were generated from cells treated with vehicle, $100 \mathrm{nM}$ torin 1 for 6 h, or $20 \mathrm{nM}$ apilimod for 3 or $6 \mathrm{~h}$. Representative images are shown with corresponding molecular weights $(\mathrm{kDa})$ shown to the right of the blots. (C) Quantification of lysosomal protein levels from corresponding blots shown in B. Shown is the mean abundance \pm SEM from four independent experiments as compared against HSP60 and normalized against the vehicle-treated control. Asterisks $(*)$ indicate statistical difference compared to the vehicle-treated control $(\mathrm{p}<0.05)$ using multiple Student's t-test with Bonferroni correction. 
A
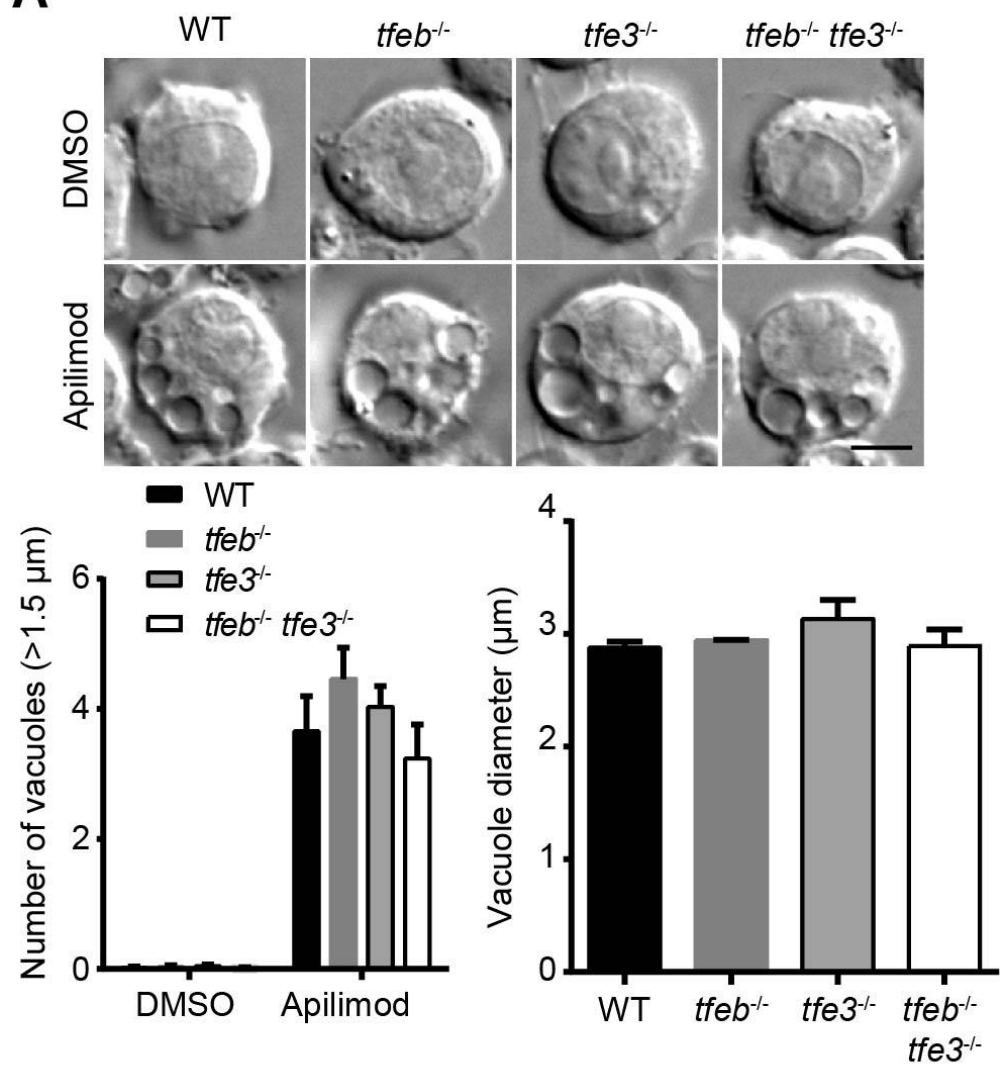

B
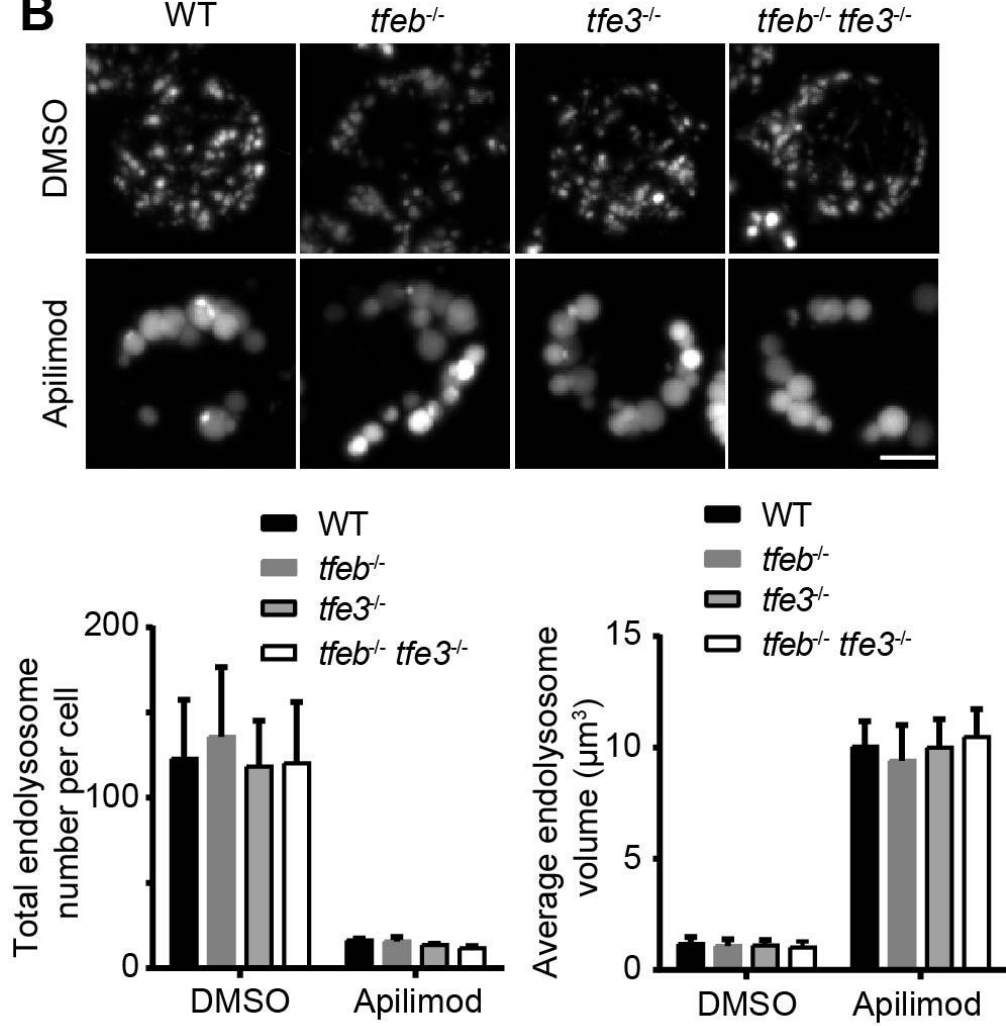
Figure 3.1.9: Lysosome enlargement during PIKfyve inhibition is not abated by $t f e b^{-/-}$and or $t f e 3^{-/-}$. Lysosomes number and size are quantified in wild type, $t f e b^{-/-}, t f e 3^{-/-}$, or $t f e b^{-/-} t f e 3^{-/-}$RAW macrophages. (A) Manual quantification of vacuoles in RAW cells. Cells treated with vehicle or $20 \mathrm{nM}$ apilimod for $1 \mathrm{~h}$ were imaged live with DIC optics. Here, vacuoles are defined as visible lysosomes whose diameter is equal or greater than $1.5 \mu \mathrm{m}$. The accompanying quantifications count the average number of vacuoles \pm SEM per cell or the average diameter of vacuoles \pm SEM within cells from three independent experiments. (B) Automated counting of lysosome size in RAW cells. Lysosomes were preloaded with lucifer yellow and cells were treated with either vehicle or $20 \mathrm{nM}$ apilimod for $1 \mathrm{~h}$ before live-cell imaging. Lysosome number and size are quantified using Icy automated bioimaging analysis software. Quantifications show the average number of lysosomes \pm SEM per cell or the average volume of lysosomes \pm SEM per cell from five independent experiments. Statistical tests were performed using one-way ANOVA comparing knockout backgrounds against the wild-type background. Scale bars indicate $5 \mu \mathrm{m}$. 


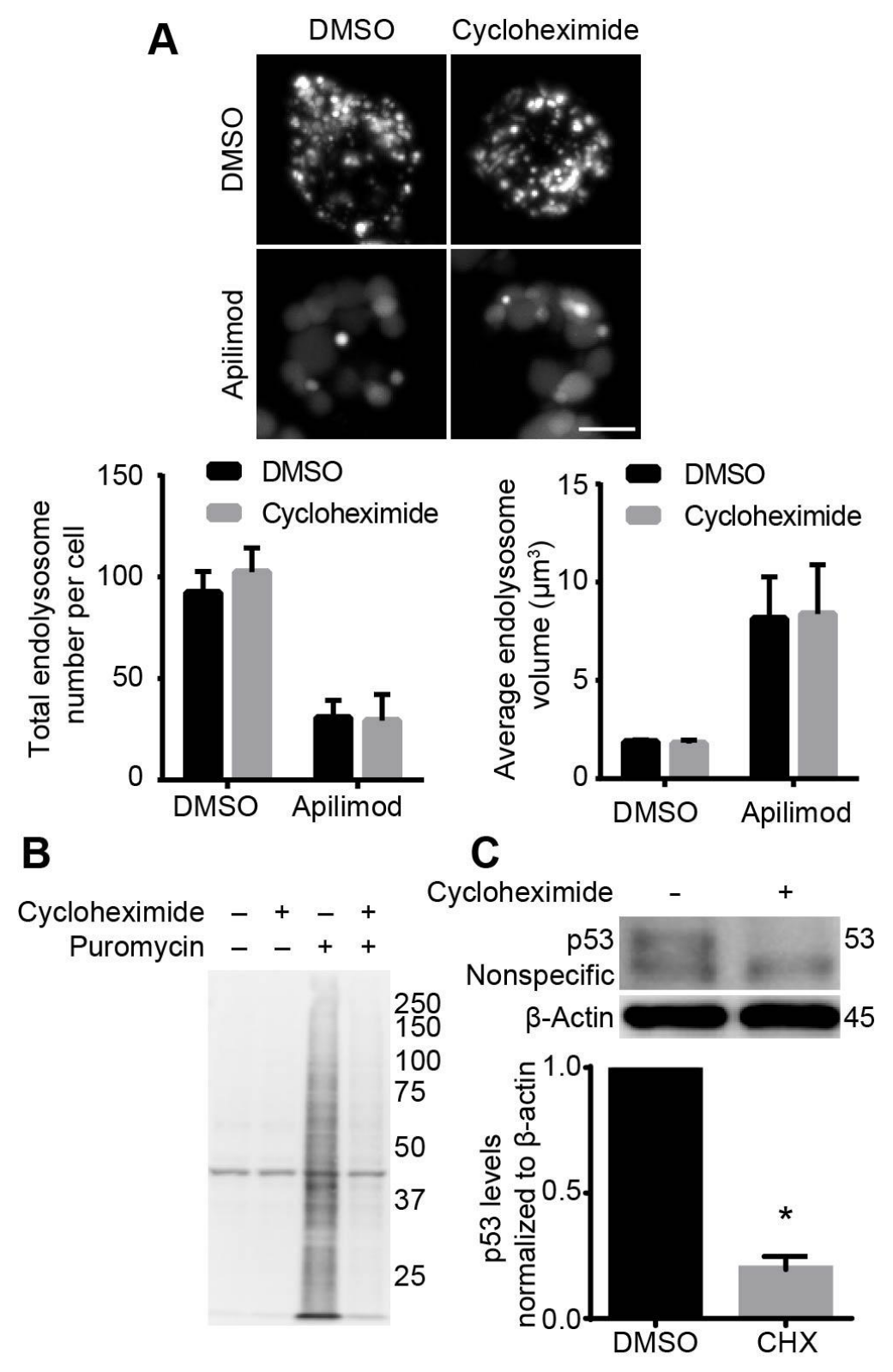


Figure 3.1.10: Protein synthesis does not contribute to lysosome enlargement during PIKfyve inhibition. (A) Morphology of lysosomes in RAW cells pre-labeled with lucifer yellow. Cells were treated with $10 \mu \mathrm{M}$ cycloheximide or vehicle for $1 \mathrm{~h}$, then treated with vehicle or $20 \mathrm{nM}$ apilimod for $1 \mathrm{~h}$. Quantifications show the average number of lysosomes \pm SEM per cell or the average volume of lysosomes \pm SEM per cell from three independent trials. Statistical tests were performed using Student's t-test comparing cycloheximide- and vehicle-treated cells. Scale bar indicates $5 \mu \mathrm{m}$. (B, C) Confirmation of protein synthesis inhibition with cycloheximide. (B) Western blot analysis of puromycin incorporation into actively synthesizing proteins. RAW cells are treated with either vehicle or $10 \mu \mathrm{M}$ cycloheximide for $1 \mathrm{~h}$ with the addition of $10 \mu \mathrm{g} / \mathrm{mL}$ puromycin $15 \mathrm{~min}$ before cell lysis. Shown is a representative image from three independent experiments. (C) Western blot analysis of fast-turnover protein p53 with cycloheximide inhibition from whole cell lysates. Representative image is shown of three independent experiments with molecular weights $(\mathrm{kDa})$ shown to the right of the blot. Cells were treated with vehicle or $10 \mu \mathrm{M}$ cycloheximide for $1 \mathrm{~h}$. The accompanying quantification shows the mean abundance of $\mathrm{p} 53$ relative to $\beta$-actin, normalized to the vehicle-treated control sample. Asterisks (*) indicate statistical difference compared to the vehicle-treated control $(\mathrm{p}<0.05)$ using multiple Student's t-test with Bonferroni correction. 

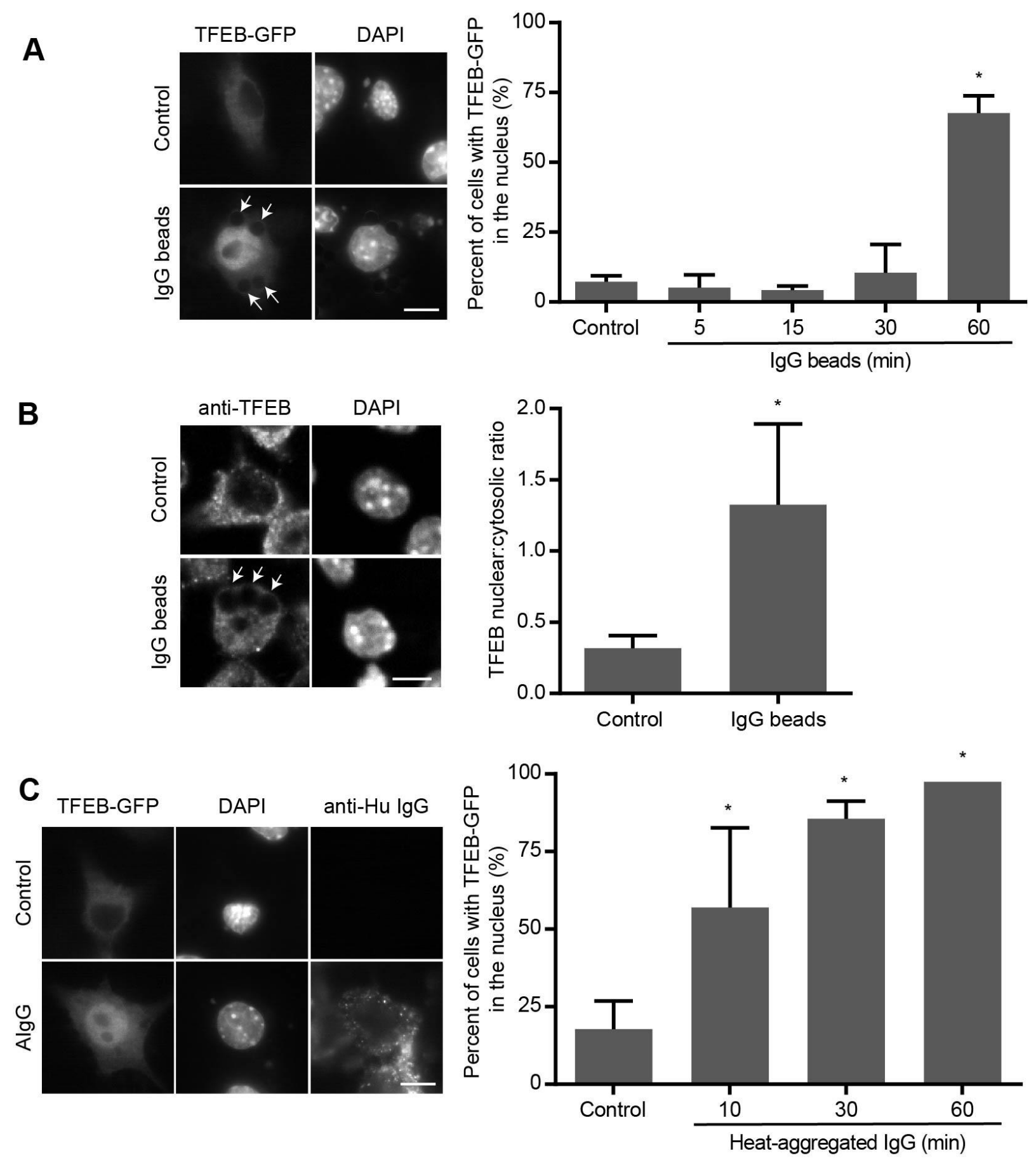
Figure 3.2.1: Engagement of the Fc $\gamma$ receptor induces TFEB nuclear localization. (A, B) RAW cells expressing TFEB-GFP (A) or stained for endogenous TFEB (B) were stimulated with IgGopsonized beads. Beads were chased for 60 min unless otherwise indicated. Arrows indicate internalized beads. (C) RAW cells expressing TFEB-GFP were also challenged with heataggregated $\operatorname{IgG}(\mathrm{AIgG})$. Cells were fixed after the chase times indicated. The nucleus was stained with DAPI where indicated. Nuclear localization of TFEB was expressed as a percent of cells with greater mean fluorescence in the nucleus compared to the cytosol of TFEB-GFP (A, C) or the nuclear-to-cytosolic mean fluorescence intensity ratio for endogenous TFEB (B).

Quantifications shown are the mean \pm SEM for three independent experiments with at least 30 cells counted per condition per experiment. Asterisks (*) indicate statistical difference compared to the vehicle treated control $(\mathrm{p}<0.05)$ using one-way ANOVA with Tukey's post-hoc test $(\mathrm{A}, \mathrm{B})$ or Student's t-test (C). Scale bars indicate $5 \mu \mathrm{m}$. 


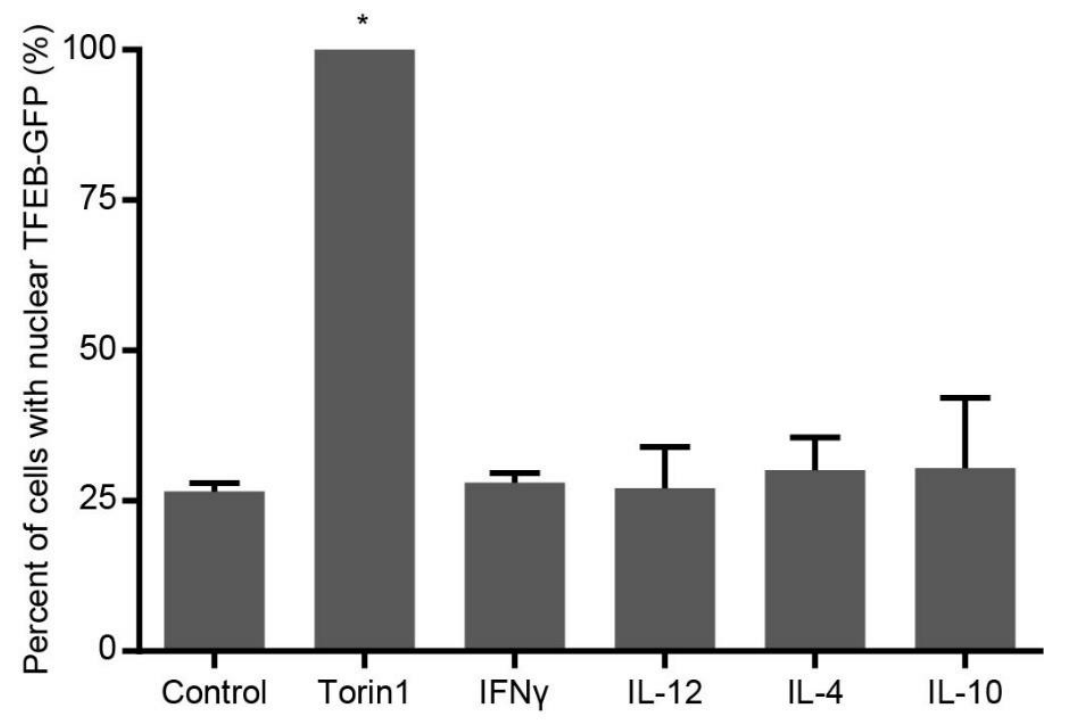


Figure 3.2.2: Cytokine treatment does not induce TFEB nuclear translocation. RAW cells expression TFEB-GFP were stimulated with INF $\gamma$, IL-12, IL-40, or IL-10 for 4 h. Quantification shown represents the percent of cells with greater mean fluorescence in the nucleus compared to the cytosol of TFEB-GFP. Data shown is the mean \pm SEM from three independent experiments with at least 30 cells counted per condition per experiment. Cells were treated with $100 \mathrm{nM}$ torin 1 for $1 \mathrm{~h}$ as a positive control. Asterisks $(*)$ indicate statistical difference compared to the vehicle-treated control $(\mathrm{p}<0.05)$ using one-way ANOVA with Tukey's post-hoc test. 

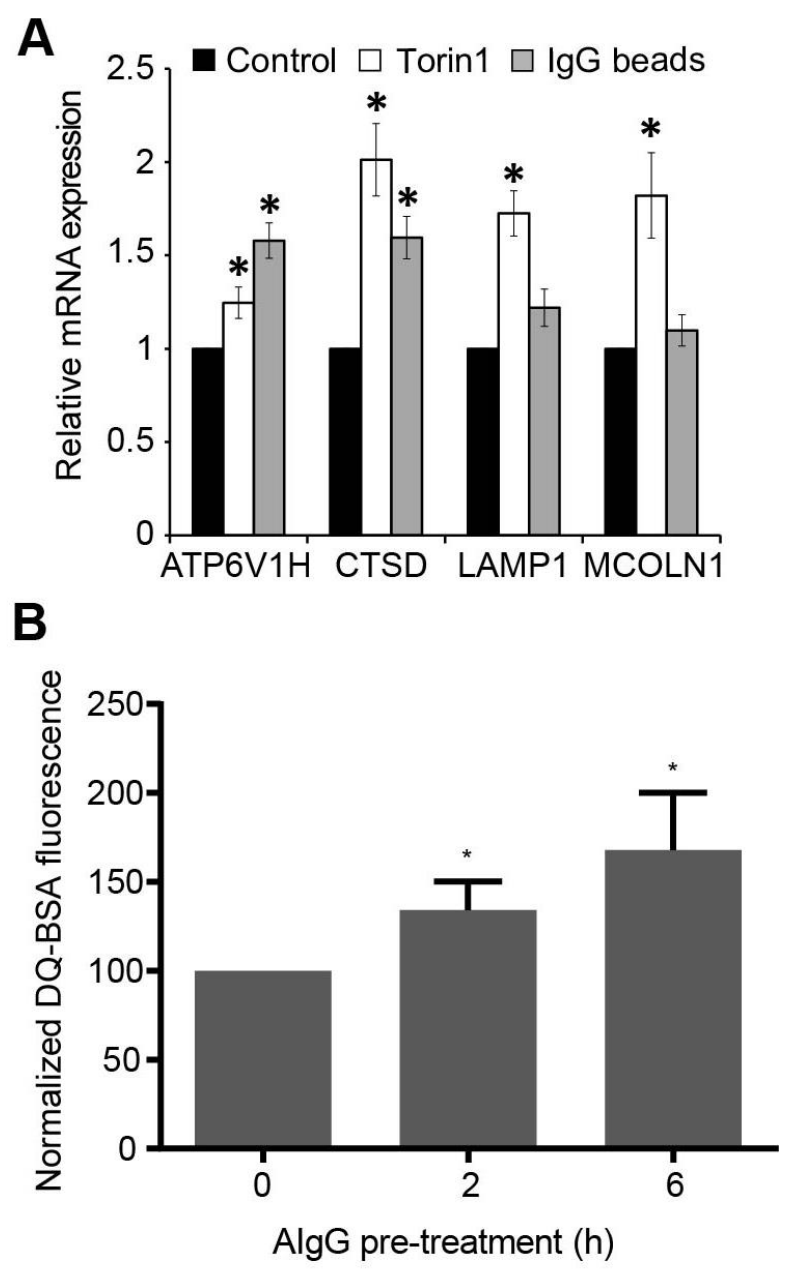
Figure 3.2.3: TFEB activation with Fc $\gamma$ receptor engagement enhances lysosome proteolysis. (A) Quantification of select lysosomal genes by qRT-PCR. Total RNA was extracted from basal cells or cells challenged with IgG-opsonized beads for $4 \mathrm{~h}$. Torin1 was used as a positive control at $100 \mathrm{nM}$ for $4 \mathrm{~h}$. Shown is the mean abundance \pm SEM relative to the control gene ABT1 and normalized against the vehicle-treated control condition from four independent trials. (B) DQBSA proteolysis following Fc $\gamma$ receptor engagement. RAW cells were challenged with heataggregated IgG (AIgG) for the times indicated to pre-active TFEB and to stimulate lysosome biogenesis. Following treatment, cells co-endocytosed DQ-BSA and dextran as a control for internalization. Cells were allowed to degrade the BSA for $1 \mathrm{~h}$. Quantification shown represents the mean fluorescence \pm SEM of DQ-BSA normalized to dextran by flow cytometry, from three independent experiments. Asterisks (*) indicate statistical difference compared to the control condition or no IgG stimulation condition $(\mathrm{p}<0.05)$ using one-way ANOVA with Tukey's posthoc test. 

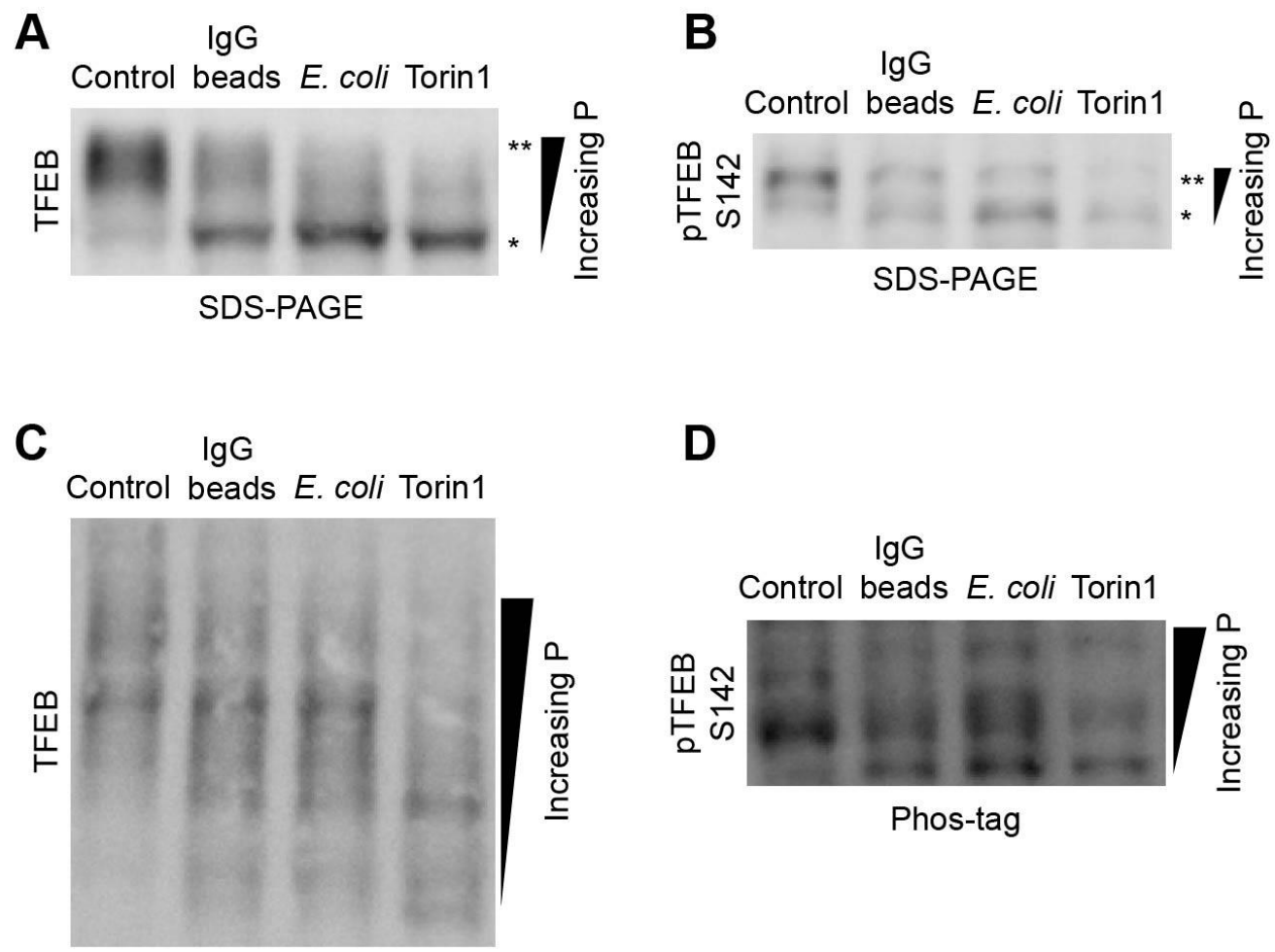

D

Phos-tag

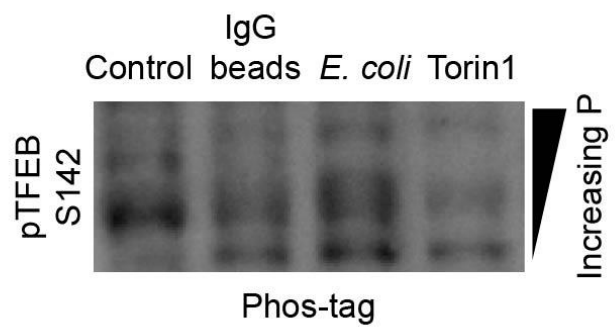


Figure 3.2.4: TFEB phosphorylation changes following phagocytosis. Western blot of whole cell lysates after phagocytosis of IgG-opsonized beads or E. coli for $1 \mathrm{~h}$. Lysates were run on standard SDS-PAGE gels (A, B) or SDS-PAGE gels with Phos-tag and $\mathrm{Mn}^{2+}$ (B, C). TFEB mobility shift was analyzed by looking at total TFEB (A, C) or phospho-TFEB at S142 (B, D). Mobility shift in phagocytosis conditions are compared against basal cells, or against cells treated with $100 \mathrm{nM}$ torin1 for $1 \mathrm{~h}$ as a positive dephosphorylation control. Representative blots are shown from 3 independent experiments. 

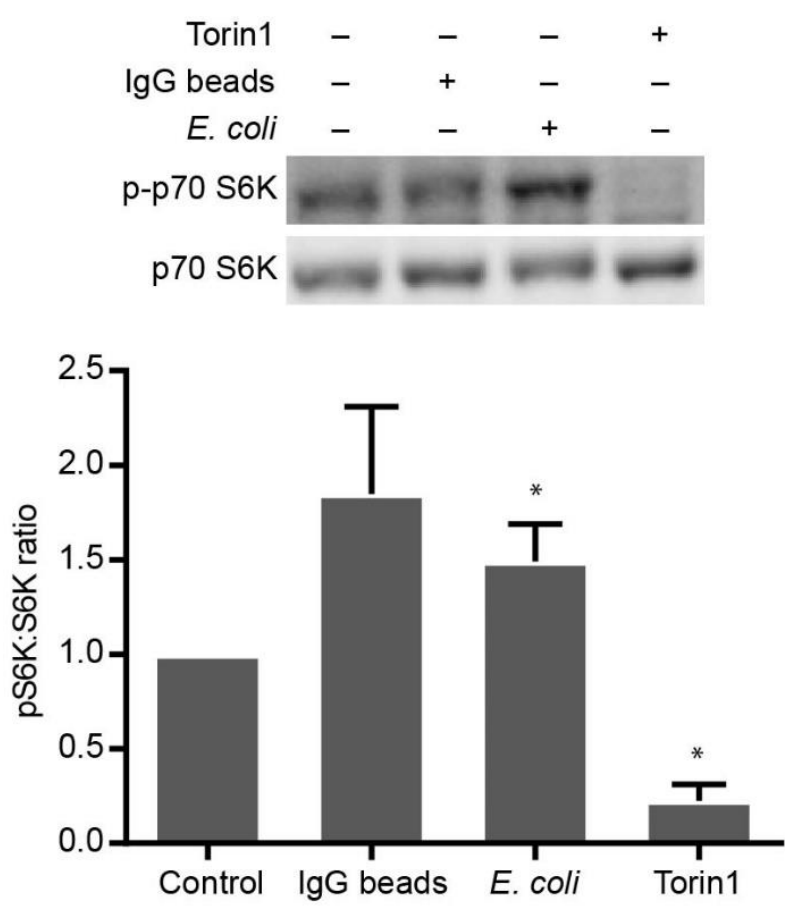
Figure 3.2.5: mTORC1 activity does not decrease following phagocytosis. mTORC1 activity was reported by phosphorylation of p70-S6K. Cells treated with either IgG-opsonized beads, E. coli, or torin 1 as a positive control. Shown is a representative image from three independent experiments. The accompanying quantification shows mean abundance \pm SEM of phospho-p70S6K at T389 compared to total p70-S6K, and normalized against the basal control. Asterisks $\left(^{*}\right)$ indicate statistical difference compared to the control condition $(\mathrm{p}<0.05)$ using multiple Student's t-tests with Bonferroni correction. 

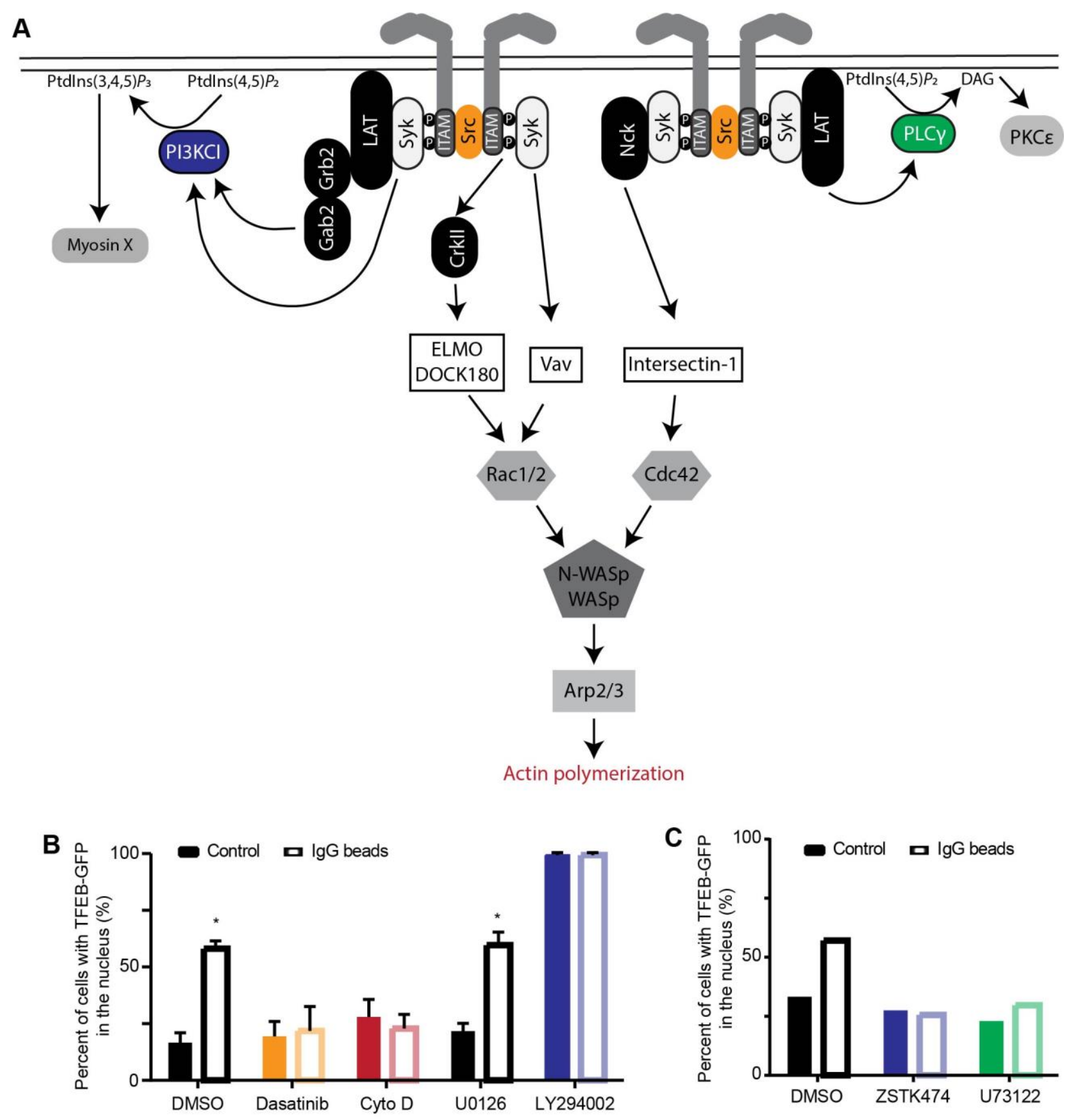
Figure 3.2.6: Fc $\gamma$ receptor signaling appears necessary for TFEB nuclear localization. (A) Fc $\gamma$ receptor signaling leads to a complex and multifaceted pathway that culminates in actin polymerization. (B, C) RAW cells expressing TFEB-GFP were pre-treated with the inhibitors indicated for 10-30 min prior to phagocytosis. The targets of the respective inhibitors are colourcoded in A, except ERK inhibited by U0126. Cells were then challenged with inhibitor alone (solid colour bar) or inhibitor with IgG-opsonized beads (bar outlined). (B) Quantifications show the mean \pm SEM percent of cells with greater TFEB-GFP nuclear fluorescence than in the cytosol for three independent experiments. Asterisks (*) indicate statistical difference compared to the basal, inhibitor only control $(\mathrm{p}<0.05)$ using Student's t-test. (C) Quantifications show the percent of cells with greater nuclear TFEB-GFP fluorescence compared to cytosolic fluorescence from one experiment. 


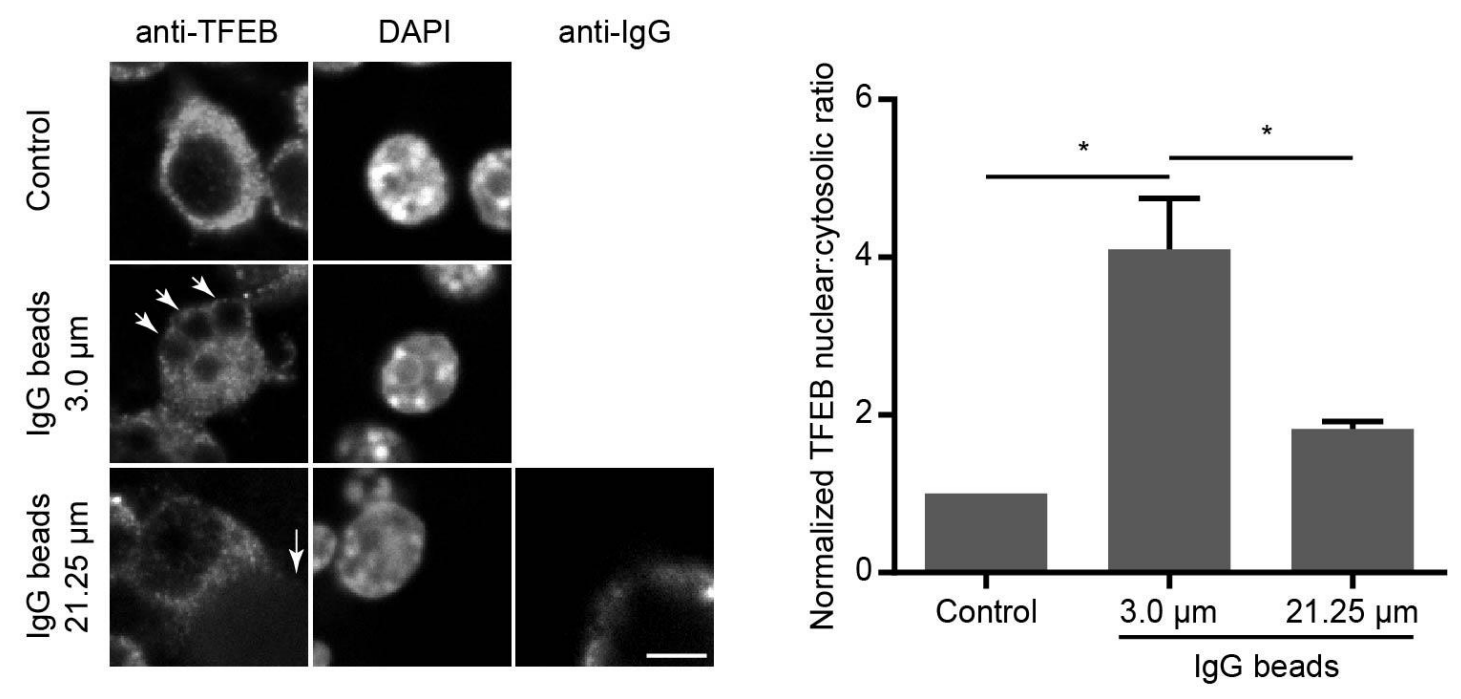


Figure 3.2.7: Particle internalization is required for TFEB nuclear localization. Representative images of endogenous TFEB after RAW cells were challenged with 3.0 or 21.25 IgG-opsonized beads. Where indicated, DAPI is used to stain the nucleus and secondary anti-human IgG was used to visualize the external beads. The corresponding quantification shown the mean TFEB nuclear-to-cytosolic ratio \pm SEM from thee independent experiments. Asterisks $(*)$ indicate statistical difference between the two conditions under the horizontal line $(\mathrm{p}<0.05)$ using Student's t-test. 


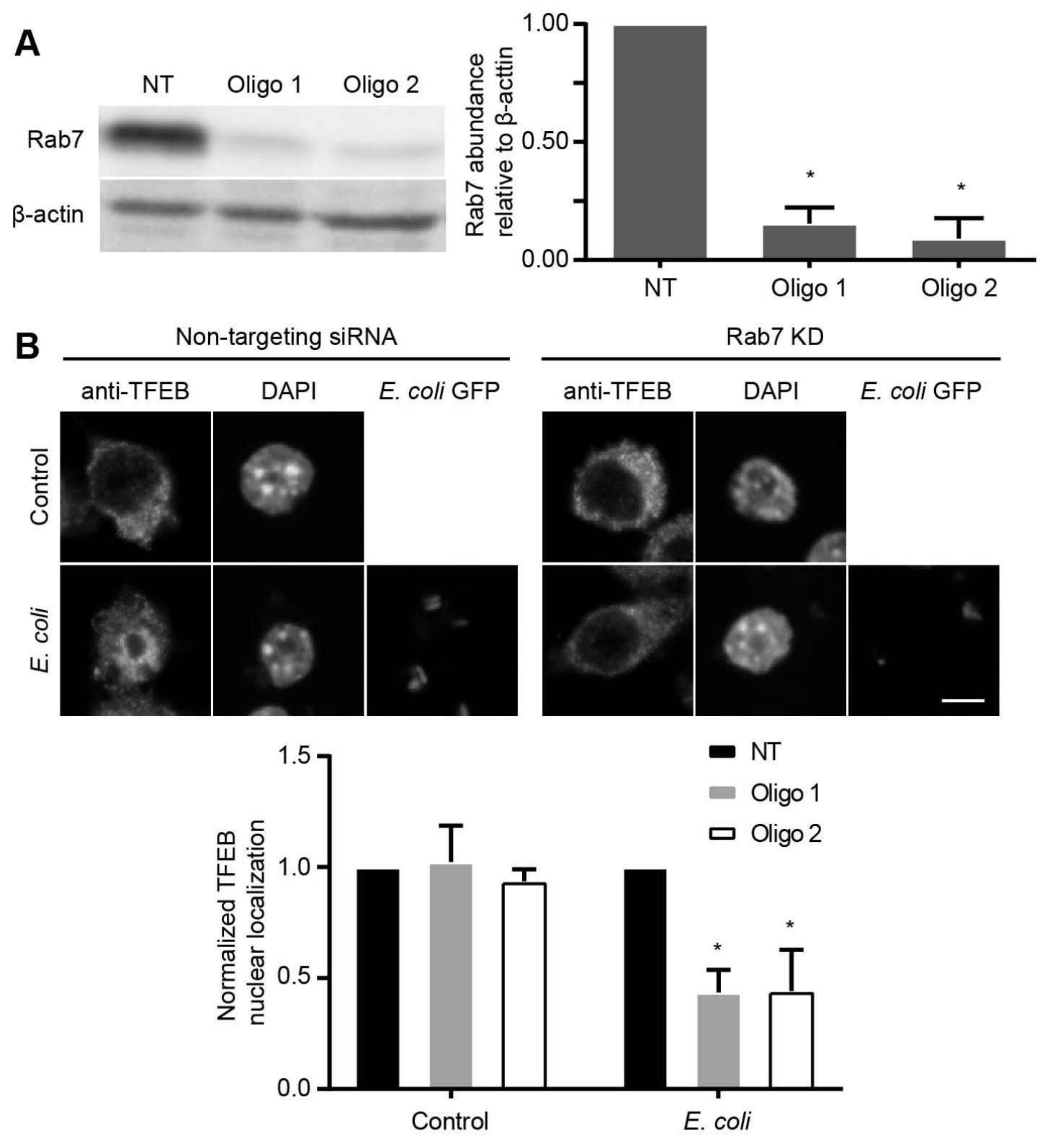


Figure 3.2.8: Rab7 is required for TFEB nuclear localization with E. coli. (A) Confirmation of Rab7 knockdown. Rab7 was knocked-down with two rounds of gene silencing with two separate siRNA oligos against Rab7 or with non-targeting siRNA. The blot shown is a representative image from three independent experiments. The accompanying quantification shows the mean abundance \pm SEM of Rab7 protein silenced with either Rab7 oligo 1, 2 or non-targeting siRNA. (B) Rab7 knockdown prevents TFEB nuclear localization with E. coli but not IgG-opsonized beads. RAW cells knocked down for Rab7 or non-targeting siRNA were challenged with IgGopsonized beads or E. coli and stained for endogenous TFEB. Where indicated, DAPI was used to stain the nucleus or the phagocytic particle were fluorescently illuminated. Representative images are shown from three independent experiments. Quantification shown represents the mean TFEB nuclear-to-cytosolic ratio \pm SEM by normalizing the ratio against the basal cells and then against the non-targeting control, from three independent experiments. Asterisks (*) indicate statistical difference between Rab7-knockdown compared to the non-targeting control $(\mathrm{p}<0.05)$ using one-way ANOVA with Tukey's post-hoc test or multiple Student's t-test with Bonferroni correction. Scale bar represents $5 \mu \mathrm{m}$. 
Chapter 4: Discussion 
Increasing lysosome biogenesis is an adaptive strategy adopted by cells under various forms of cellular stress, including starvation, oxidative stress and immune signaling (Martina \& Puertollano, 2018; Najibi et al., 2016; Roczniak-Ferguson et al., 2012). Enhancing lysosome biogenesis and related trafficking pathways also has therapeutic value in a variety of diseases stemming from insufficient autophagy and molecular degradation (Palmieri et al., 2017; Spampanato et al., 2013). Lysosome biogenesis can be controlled by activating TFEB through overexpression or altering phosphorylation. To date, six kinases and two phosphatases are responsible for altering TFEB phosphorylation (Martina \& Puertollano, 2018; Puertollano et al., 2018). Many phosphorylation events alter TFEB localization, modulated by kinases like mTORC1, ERK2, Akt, MAP4K3 and GSK3 $\beta$, and phosphatases like calcineurin and PP2A (Figure 1.1.2, black circles with P). Additionally, TFEB stability can be altered by phosphorylation by PKC $\beta$ (Figure 1.1.2, grey circles with P). Here, we identified two additional pathways that can induce nuclear localization and activation of TFEB: PIKfyve-inhibition induced lysosome enlargement and Fc $\gamma$ receptor mediated phagocytosis.

\subsection{PIKfyve regulation of TFEB}

\subsubsection{TFEB is not required for PIKfyve inhibition-induced lysosome enlargement}

$\operatorname{Ptd} \operatorname{Ins}(3,5) P_{2}$ is necessary for maintenance of numerous lysosomal functions including autophagosome degradation, ion homeostasis, and maintenance of lysosome size (Dong et al., 2010; Cole J. Ferguson et al., 2009; Ikonomov, Sbrissa, \& Shisheva, 2001). The most obvious defect due to PtdIns $(3,5) P_{2}$ depletion is the dilation of the lysosome, a process that remains incompletely understood. To date, the prevailing model suggests lysosome enlargement is due to a failure for membranes to fission from the endolysosomal compartment (Bryant et al., 1998; 
Krishna et al., 2016; Rutherford, 2006). Chief among these is the defect in retrograde traffic, necessary for the retrieval of the mannose 6-phosphate receptor from the endosome to the transGolgi network (Rutherford, 2006). However, enhanced biosynthesis of lysosomes via TFEB and/or related family members activation has yet to be evaluated.

Our results show that PIKfyve inhibition induced nuclear localization of TFEB (Figure 3.1.2) and its family members, TFE3 and MiTF (Figure 3.1.3). Consistently, others have also shown that PIKfyve inhibition induced TFEB nuclear localization in several cell types (Gayle et al., 2017; W. Wang et al., 2015). Furthermore, nuclear TFEB correlated with transcriptional upregulation of several lysosomal genes (Figure 3.1.8A). Interestingly, the increase in transcription did not lead to enhanced translation over 6 hours, aside from LAMP1 (Figure 3.1.8B,C). This disconnect between transcription and translation may be due to multiple trafficking defects resulting from PIKfyve inhibition. Chronic PIKfyve inhibition impaired recycling of the mannose 6-phosphate receptor from the endosome to the trans-Golgi network, leading to degradation of mannose 6-phosphate receptor (Rutherford, 2006; Y. Zhang et al., 2007). Correspondingly, inactive pro-forms of cathepsin proteases appear to accumulate during prolonged PIKfyve inhibition (Gayle et al., 2017; Y. Zhang et al., 2007). Additionally, PIKfyveinhibited cells are thought to have impaired autophagosome turnover and increased levels of LAMP2 (Cole J. Ferguson et al., 2009), which may correlate with LAMP1 detected at 6 hours (Figure 3.1.8B,C). Nevertheless, impaired biosynthesis did not abate lysosome enlargement. TFEB and family members appear to be dispensable for lysosome swelling during PIKfyve inhibition when measuring lysosome number and size (Figure 3.1.9, A1.2). Similarly, blocking translation with cycloheximide also did not abate lysosome swelling (Figure 3.1.10). Together, 
these results suggest lysosome enlargement during acute PIKfyve inhibition do not depend on biosynthesis.

\subsubsection{PIKfyve regulation of mTORC1}

TFEB activity is understood to be directly regulated by at least six kinases and two phosphatases (Martina \& Puertollano, 2018; Puertollano et al., 2018). Loss of phosphorylation is most often associated with nuclear translocation of TFEB. Consistent with this idea, TFEB nuclear localization during PIKfyve inhibition correlated with a loss of phosphorylation, as judged by increased TFEB mobility through Phos-tag gels (Figure 3.1.4). Increased TFEB mobility has also been observed by standard SDS-PAGE gels during PIKfyve inhibition (Gayle et al., 2017). While the Phos-tag assay indicated a decrease in the number of TFEB phosphorylations, it does not provide any information regarding the identity of the residues being modified. Knowing the residues being altered would hint at specific kinases or phosphatases responsible for TFEB dephosphorylation. To this end, very few TFEB phospho-specific antibodies have been generated and only one is commercially available (Settembre et al., 2012; Vega-Rubin-de-Celis et al., 2017). Thus, we assessed the activities of known kinases to determine their relative contributions to TFEB dephosphorylation during PIKfyve inhibition.

The most well-characterized TFEB kinase is mTORC1, which directly modulates S211 phosphorylation. This phospho-site forms a docking site for 14-3-3 which physically masks the nuclear localization sequence upon binding, keeping TFEB inactive and in the cytosol (Roczniak-Ferguson et al., 2012). We postulate that upstream regulators of mTORC1 may also regulate TFEB function. PIKfyve function appears to be necessary for mTORC1 activation in 
adipocytes (Bridges et al., 2012). Thus, we examined a possible PIKfyve-mTORC1-TFEB pathway. Contrary to this idea, mTORC1 appeared to be active during PIKfyve inhibition when examining the phosphorylation of S6K. These results are consistent with others who show that S6K remains phosphorylated during PIKfyve inhibition (Krishna et al., 2016; W. Wang et al., 2015).

We also evaluated the possibility that mTORC1 controls PIKfyve. In this hypothetical pathway, PtdIns $(3,5) P_{2}$ may recruit TFEB to the lysosome similar to recruitment of Tup1 in yeast during galactose metabolism (Han \& Emr, 2011). Here, mTORC1 inhibition may reduce PIKfyve activity and reduce PtdIns(3,5) $P_{2}$ levels, preventing TFEB recruitment and subsequent phosphorylation. However, PtdIns $(3,5) P_{2}$ levels were not reduced during mTORC1 inhibition (Figure 3.1.5).

Together, these results suggest PIKfyve regulation of TFEB acts in parallel to mTORC1. However, we cannot discount the possibility of functionally distinct pools of mTORC1 that may act differently on TFEB and S6K. For example, mTORC1 is thought to be most active when lysosomes are localized peripherally, where Rheb is proximal to growth factor signaling for activation (Korolchuk et al., 2011). Additionally, mTORC1 may act on different pools of substrates differently based on rapamycin inhibition of mTORC1, which has been attributed to substrate affinity to mTORC1 (Kang et al., 2013). Substrates like TFEB and 4E-BP1 are rapamycin insensitive substrates while S6K becomes dephosphorylated in the presence of rapamycin (Settembre et al., 2012; Thoreen et al., 2009). Thus, it may be possible that a PtdIns(3,5)P $P_{2}$ sensitive pool of mTORC1 regulates TFEB while S6K is regulated by mTORC1 is $\operatorname{PtdIns}(3,5) P_{2}$ is insensitive. 
Similarly, there may also be specific pools of $\operatorname{PtdIns}(3,5) P_{2}$ that may activate TFEB aside from the bulk PtdIns(3,5)P . HPLC-coupled flow scintillation allows for measurement of total PtdIns(3,5) $P_{2}$, but does not discriminate between localization or acyl chain content. Thus, we can only conclude that PtdIns $(3,5) P_{2}$ did not decrease. However, specific pools of $\operatorname{Ptd} \operatorname{Ins}(3,5) P_{2}$ may exist. For example, the plasma membrane associated PtdIns(3,5) $P_{2}$ observed by Bridges et al. was synthesized by PI3K-C2 $\alpha$-PIKfyve pathway, rather than the canonical Vps34-PIKfyve pathway (Bridges et al., 2012). Another possibility is that PI5P is responsible for TFEB recruitment. PI5P is thought to be primarily generated by dephosphorylation of $\operatorname{PtdIns}(3,5) P_{2}$, which would also depend on active PIKfyve (Oppelt et al., 2014; Zolov et al., 2012).

\section{$\underline{\text { 4.1.3 PIKfyve regulation of other known TFEB kinases }}$}

We further investigated TFEB activation by other known kinases. ERK2 and GSK3 $\beta$ are thought to phosphorylated TFEB at S142 and S134/S138 respectively (Y. Li et al., 2016; Settembre et al., 2011). While we did not observe a difference in ERK2 phosphorylation with apilimod-treatment (Figure 3.1.6B), we did see an increase in GSK3 inhibitory phosphorylation, of both isoforms (Figure 3.1.7). GSK3 preferentially binds "primed" substrates that are prephosphorylated four residues C-terminal to the site of phosphorylation. Increased GSK3 phosphorylation at S21 for GSK3 $\alpha$ and S9 for GSK3 $\beta$ act as pseudo-substrates and are more likely to block the active site of the kinase, leading to inhibition (Frame et al., 2001). Therefore, we overexpress a constitutively active GSK3 $\beta$-S9A mutant, preventing the active site from binding its pseudo-substrate (Stambolic \& Woodgett, 1994). Cells expressing wild-type GSK3 $\beta$ or GSK3 $\beta-S 9 A$ did not prevent TFEB nuclear localization in the presence of apilimod (Figure A1.1). 
However, we cannot conclude that GSK3 $\beta$ does not act on TFEB during PIKfyve inhibition. Activity of GSK3 $\beta$ is also dependent on phosphorylation of Y216 (Hughes, Nikolakaki, Plyte, Totty, \& Woodgett, 1993). Phosphorylation of Y216 may depend on autophosphorylation during synthesis (Cole et al., 2004) or by other kinases like Src (Goc et al., 2014). Also, while we overexpress wild-type GSK3 $\beta$ and GSK3 $\beta$-S9A, the endogenous GSK3 $\beta$ is still present in these cells. Additionally, GSK3 $\beta$ has been reported in the cytosol and in the nucleus (Bechard \& Dalton, 2009). TFEB phosphorylation by GSK3 $\beta$ was shown to occur in the nucleus where TFEB primed with phosphorylation at S142 is recognized by GSK3 $\beta$ (L. Li et al., 2018). This leads to phosphorylation of S138 which activates a nuclear export sequence on TFEB (Y. Li et al., 2016). Finally, GSK3 $\alpha$ may also act redundantly with GSK3 $\beta$ since both are inactivated in PIKfyve-inhibited cells. Thus, further investigation must be done to understand the activation, localization, and relative abundance of GSK3 $\beta$-S9A in RAW macrophages in response to PIKfyve inhibition.

While GSK3 $\beta$ may be required for nuclear exit, TFEB may also require additional signals to induce nuclear import during PIKfyve inhibition. To fully understand how TFEB regulation occurs, proteomic analysis via mass spectrometry would be an invaluable tool to determine changes to post-translational modifications. Individual bands from Phos-tag assay could be cut out and analysed for differences in phosphorylation, which would provide clues about which kinases or phosphatases are necessary for TFEB nuclear localization.

\subsubsection{Perspective}

Overall, TFEB activation during PIKfyve inhibition may be an attempted adaptive response to lysosome dysregulation. While not necessary for initial lysosome enlargement, 
TFEB activation in chronically PIKfyve-inhibited cells may occur to activate membrane trafficking pathways and lysosome biogenesis, similar to those observed in lysosome storage disorders (Medina et al., 2011; Spampanato et al., 2013). However, these attempts may be futile since PIKfyve appears to be necessary for biosynthetic traffic of lysosome luminal proteins, leading to build up of inactive lysosomal proteins (Gayle et al., 2017). Therefore, increasing lysosome biogenesis may induce further stress on these cells. Alternatively, PIKfyve-regulation

of TFEB may constitute a feedback pathway that cannot be dissected by inhibiting PIKfyve due to pleiotropic effects. As noted above, proteomic studies on TFEB in PIKfyve-inhibited cells may provide target sites to do site-directed mutagenesis to better understand the role of TFEB downstream of PIKfyve. In addition, transcriptomic studies in PIKfyve-inhibited cells in wildtype and TFEB/TFE3-deleted cells may provide clues about the relationship between these two proteins.

\subsection{TFEB regulation by phagocytosis}

\subsubsection{Phagocytosis activates TFEB to enhance proteolysis}

Phagocytosis is a necessary component of immune function, sequestering pathogens into phagosomes. These phagosomes undergo maturation and subsequent fusion with the lysosome for degradation. Impaired degradation of cargo can prevent further phagocytosis of pathogens (Wong et al., 2017). Thus, phagocytes may benefit from increasing lysosome biogenesis through TFEB activation to accelerate phagosome breakdown and neutralization of potential threats. Here we show that $\mathrm{Fc} \gamma$ receptor activation induced TFEB nuclear localization (Figure 3.2.1). Phagocytosis of IgG-opsonized beads or endocytosis of heat-aggregated IgG were both sufficient to activate TFEB. Interestingly, $\mathrm{Fc} \gamma$ receptor-mediated phagocytosis selectively upregulated 
some lysosomal genes, but not all (Figure 3.2.3A). Compared to mTORC1 inhibition with torin1 which upregulated all lysosomal genes tested (Figure 3.2.3, torin1), phagocytosis of IgGopsonized beads selectively upregulated mRNA levels of ATP6V1H and protease CTSD, but not membrane protein $L A M P 1$ or calcium channel MCOLN1 (Figure 3.2.3, IgG beads). Protein abundance of these genes (Figure A2.1A) agreed with the qRT-PCR data. This data suggests that Fc $\gamma$ receptor activation does not induce complete lysosome biogenesis leading to more lysosomes. Instead, existing lysosomes were augmented and became "super active.” Increased proteolysis and acidification of the lysosome was also observed in dendritic cells (Samie \& Cresswell, 2015). Additionally, we observed no difference in the number of LAMP1 positive structures between cells stimulated with heat-aggregated IgG and control cells (Figure A1.3), further strengthening our hypothesis.

The selective increase in lysosome gene transcription may be attributed to other transaction factors, miRNA, and/or modifications to TFEB in the nucleus. As opposed to TFEB, ZKSCAN3 is a transcriptional repressor in fed-cells, responsible for regulating over 60 lysosomal genes (Chauhan et al., 2013). When cells undergo starvation, ZKSCAN3 exits the nucleus at the same time TFEB enters the nucleus to upregulate CLEAR gene transcription (Chauhan et al., 2013). Other transcription factors may also be activated in response to phagocytosis, such as NF-kB that modulates cytokine release (Garc1 \& Rosales, 2001). Additionally, microRNAs are known to modulate large sets of genes and are activated in many immune cells during inflammation (O’Connell, Rao, \& Baltimore, 2012). Interestingly, TFEB is under direct regulation by miR-128 which supresses CLEAR network gene expression (Sardiello et al., 2009). Finally, TFEB can be post-translationally modified with SUMO or acetyl groups that can alter the binding of TFEB to specific promoters (Figure 1.1.2) (Bao et al., 2016; Miller 
et al., 2005; J. Zhang et al., 2018). It would be interesting to determine the potential interplay between TFEB and other regulatory factors that may impinge on TFEB activity to selectively control a subset of lysosomal genes.

Nevertheless, cells with “super active” lysosomes following Fc $\gamma$ receptor activation were more proteolytic (Figure 3.2.3B) and were better killers of live E. coli than their control counterparts (Figure A2.2). The increase in bacterial killing was dependent on TFEB as both increases to CTSD and ATP6V1H protein levels (Figure A2.1B) and bacterial killing (Figure A2.2, right side) were abated in TFEB-silenced cells. Together, these results suggest Fc $\gamma$ receptor activation, leads to TFEB-mediated hyperactivation of existing lysosomes. Aside from the Fc $\gamma$ receptor, TFEB also becomes activated when cells are challenged with unopsonized $E$. coli (Figure 3.2.8B). This is consistent with macrophage stimulation with S. aureus, leading to TFEB-dependent upregulation of pro-inflammatory cytokines (Visvikis et al., 2014). However, cytokines themselves were unable to stimulate TFEB activation (Figure 3.2.2).

Thus, it would be interesting to determine the range of phagocytic signals that stimulate TFEB activation. A particularly interesting example is the clearance of apoptotic bodies. Apoptotic cells can be recognized by receptors like Tim4 and induce anti-inflammatory activation of macrophages (Miyanishi et al., 2007; Szondy, Sarang, Kiss, Garabuczi, \& Köröskényi, 2017). Additionally, apoptotic cells can also be phagocytosed by other cell types such as epithelial cells (Monks et al., 2005). It would be interesting to see whether nonphagocytic cells also benefit from TFEB activation to accelerate degradation of phagosomes.

\subsubsection{Fc $\gamma$ receptor-to-TFEB signaling}


We also aimed to understand how TFEB is regulated during phagocytosis. Since activation of TFEB often depends on phosphorylation, we attempt to characterize changes to TFEB phosphorylation using mobility shift assays with standard SDS-PAGE gels (Figure 3.2.4 A,B) and Phos-tag gels (Figure 3.2.4 C,D). In addition to total TFEB antibody (Figure 3.2.4 A,C), we also attempt to characterize changes in S142 phosphorylation (Figure 3.2.4 B,D). In all cases, TFEB displayed faster mobility in cells that phagocytosed IgG-opsonized beads compared to resting cells. For comparison, unopsonized E. coli and mTORC1 inhibition also increased TFEB mobility, resulting in similar patterns to IgG. Interestingly, blots with pS142-TFEB antibody also shifted suggesting this phosphorylation is retained during phagocytosis (Figure 3.2.4 B,D). Analysis of mTORC1 activity via S6K phosphorylation suggests mTORC1 remains active during phagocytosis (Figure 3.2.5).

While mTORC1 inactivation may not be responsible for TFEB nuclear localization during phagocytosis, phagosome-lysosome fusion may be necessary for TFEB activation. This is supported by several lines of evidence. First, blocking Fc $\gamma$ receptor signaling leading to actin polymerization inhibited TFEB activation (Figure 3.2.6). Active Src/Syk (dasatinib, orange bars), PI3KCI (ZSTK474, blue bars), and PLC $\gamma$ (U73122, green bars) were necessary for TFEB nuclear localization. Second, phagosome formation was necessary for TFEB activation. Blocking actin polymerization with cytochalasin D also kept TFEB in the cytosol when cells were challenged with IgG-opsonized beads (Figure 3.2.6, red bars). Additionally, TFEB was cytosolic in cells unable to internalize IgG-opsonized oversized beads (Figure 3.2.7). This was consistent with cytosolic TFEB in cells unable to phagocytose IgG-opsonized coverslips (Figure A1.4B), despite Fc $\gamma$ receptor activation as reported by increase in phospho-Syk (Figure A1.4A). Finally, blocking phagosome maturation of $E$. coli by silencing Rab7 also prevented TFEB activation 
(Figure 3.2.8). Consistently, blocking phagosome-lysosome fusion by chelating $\mathrm{Ca}^{2+}$ with BAPTA-AM or siRNA knockdown of MCOLN1 also blocked TFEB nuclear localization (Figure A2.3) (Dayam et al., 2015). Given the requirement for lysosomal calcium and mucolipin-1, we speculate that activity of calcium-dependent kinases or phosphatases may altered in response to phagocytosis and lysosome-phagosome fusion to facilitate TFEB nuclear translocation.

Proteomic analysis of TFEB by mass spectrometry would provide clues regarding specific kinases or phosphatases responsible for TFEB activation during phagocytosis.

\subsubsection{Perspective}

Enhancing lysosome biogenesis appears to be an adaptive strategy utilized by phagocytes to promote degradation of phagocytic cargo. By supplementing existing lysosomes with more hydrolases and V-ATPase components, phagocytes maximize their bactericidal potential. Indeed, dendritic cells can switch between major histocompatibility complex class I (MHC-I)-mediated antigen presentation to MHC-II upon TFEB activation, associated with increased lysosomal activity and proteolytic activity (Samie \& Cresswell, 2015). With the rise of antibiotic resistant bacteria, TFEB activators may be worth investigating as a possible supplement to fight certain types of infection. Additionally, other phagocytes like neutrophils may also benefit from TFEB activation during infection. While neutrophils are generally not long-lived, they can last for at least one day after activation in vivo. Thus, transcriptional changes in neutrophils may be of relevance. Neutrophils have four lysosome-related organelles, or granules (Cowland \& Borregaard, 2016). These granules differ in their luminal content and propensity to be secreted. Since TFEB enhances exocytosis during lysosome storage (Medina et al., 2011), we speculate that TFEB activation may play a role in granule biogenesis and secretion. Thus, the spectrum of 
TFEB-mediated responses in various immune cells should be considered and investigated further.

To counter immune-cell function, many pathogens may have also evolved strategies to avoid TFEB-dependent immune responses. For example, Mycobacterium can evade the lysosome by blocking phagosome maturation. Phagosomes containing Mycobacterium fail to acquire LAMP1 and these pathogens persist within the phagosome (Clemens, Lee, \& Horwitz, 2002; Via et al., 1997). Interestingly, M. tuberculosis can modulate microRNA-33 to downregulate autophagy effectors, leading to depressed TFEB function (Ouimet et al., 2016). Similarly, live Salmonella can evade TFEB activation. Macrophages that phagocytose Salmonella fail to activate TFEB, a process dependent on several bacterial virulence factors including PhoP, SifA, and SopD (Escobar, 2017). In contrast, deletion of these virulence factors or dead-Salmonella allowed TFEB nuclear localization (Escobar, 2017). Other pathogens may manipulate TFEB for its own benefit. Human immunodeficiency virus (HIV) infection of macrophages appears to initially upregulate autophagy, promoting viral replication (Campbell, Rawat, Bruckman, \& Spector, 2015). Late in the infection, HIV can also produce negative regulatory factor (NEF), which leads to TFEB phosphorylation to prevent HIV from being degraded (Campbell et al., 2015). Other pathogens have likely evolved additional strategies to manipulate TFEB and should be investigated further. 
Chapter 5: Conclusion 
Induction of lysosome biogenesis and autophagy through TFEB-activation is an adaptive strategy adopted in response to numerous cellular stressors. Here, we identify two additional pathways that lead to TFEB activation.

First, PIKfyve inhibition leads to lysosome enlargement which may be exacerbated by TFEB-induced lysosome biogenesis. Contrary to our original hypothesis, TFEB and general biosynthesis were dispensable for acute lysosome enlargement. The specific role of TFEB during PIKfyve inhibition, whether beneficial or detrimental, remains to be seen. Nevertheless, PIKfyve inhibition appeared to be correlated with GSK3 inhibition, a known kinase of TFEB. While further work must be done to understand the role of GSK3 inhibition in this pathway, this study was the first to characterize a relationship between GSK3 and PIKfyve, to our knowledge.

Secondly, lysosome biogenesis may promote degradation of phagocytic cargo. Consistent with our initial hypothesis, phagocytosis of various immune-based particles promoted TFEB activation, enhancing proteolysis and bacterial killing. Here, the activation of TFEB appeared to depend on phagosome formation and maturation. Thus, TFEB activation may confer immunerelated benefits during immunosuppression or persistent infections. Further work should be done to identify the specific kinase or phosphatase modulating TFEB localization following phagocytosis.

Overall, our work expands on the growing list of regulators and pathways that modulate TFEB activity. Understanding the full breadth of TFEB modulators will allow for the development of novel therapeutics towards diseases that can be altered by TFEB activation or suppression. 
Appendix 


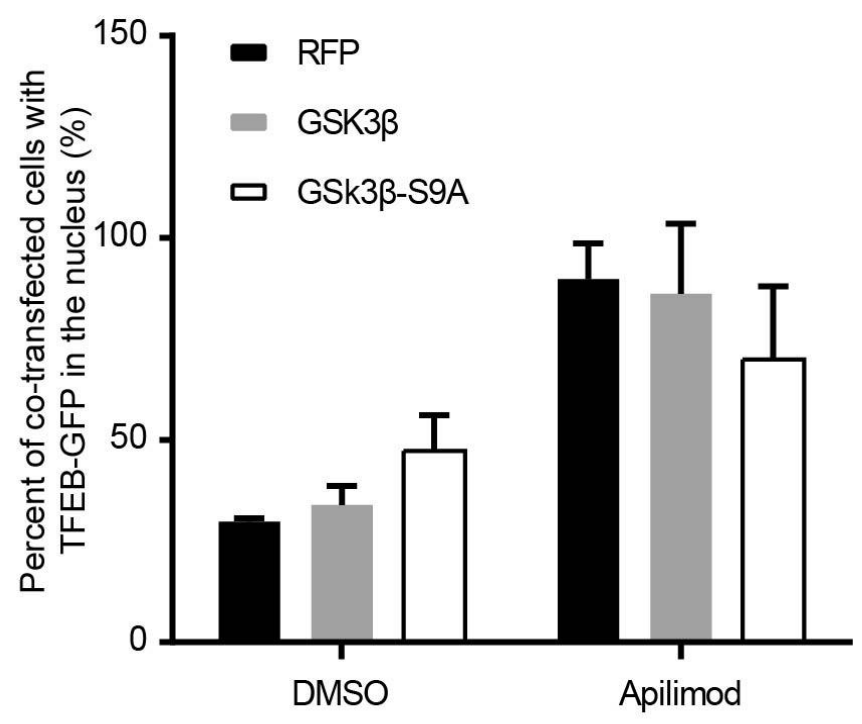


Figure A1.1: Effect of constitutively active GSK3 $\beta$ on TFEB nuclear localization. Cells coexpressed TFEB-GFP and either wild-type GSK3 $\beta$, constitutively active GSK3 $\beta$-S9A, or RFP as a transfection control. Cells were treated with vehicle or $20 \mathrm{nM}$ apilimod for 40 minutes. Quantification shown represents the mean \pm SEM percent of cells with greater nuclear TFEBGFP fluorescent intensity than the cytosol in cells co-expressing RFP or GSK3 $\beta$. Statistical tests were performed using one-way ANOVA comparing RFP- or GSK3 $\beta$-expressing cells. 

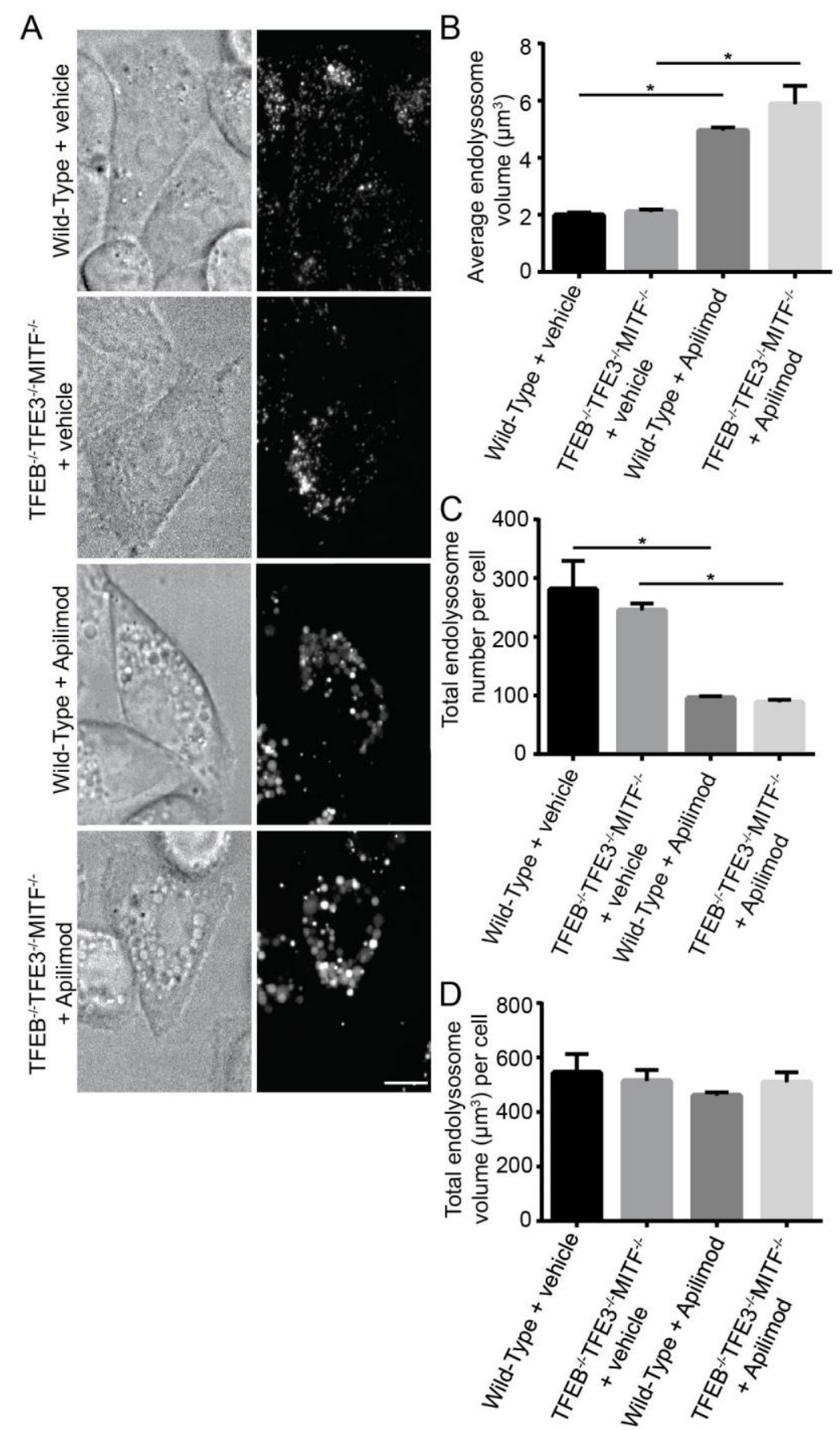
Figure A1.2: Triple knockout of MiT/TFE family members does not reduce lysosome enlargement. Lysosomes of wild type HeLa cells and HeLa cells deleted for $t f e b^{-/-}, t f e 3^{-/-}$, mitf $^{/-}$ were pre-labeled with fluorescent dextran and subjected to $50 \mathrm{nM}$ apilimod for $1 \mathrm{~h}$ or vehicle. (A) Cells were imaged live and Z-stacks were analyzed for (B) lysosome number, (C) volume of individual lysosomes, or (D) total lysosome volume. Shown is the mean \pm SEM from 3 independent experiments with 15-20 cells counted per experiment. Asterisk $\left.{ }^{*}\right)$ indicates statistical difference relative to respective controls, as shown by the horizontal bar, using $(\mathrm{p}<0.05)$ one-way ANOVA with Tukey's post-hoc test. No difference was observed between wild-type and triple knockout HeLa cells for changes during apilimod treatment. Scale bar $=10$ $\mu \mathrm{m}$. Work performed by Golam Saffi (Choy et al., 2018). 


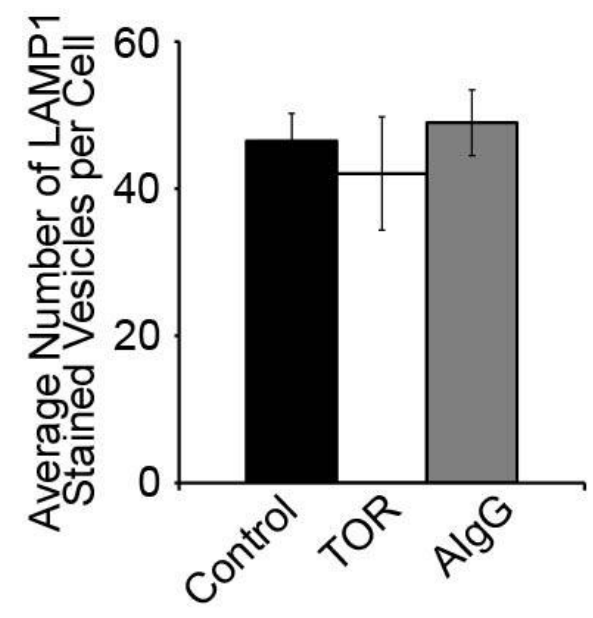


Figure A1.3: Fc $\gamma$ receptor engagement does not change lysosome number. RAW cells were stimulated with heat-aggregated $\operatorname{IgG}(\mathrm{AgG})$ for $1.5 \mathrm{~h}$, then stained for were stained for LAMP1. Z-stacks from whole cells were acquired and analyzed for number of LAMP1 positive structures. Quantification shown represents the mean number \pm SEM of LAMP1 positive structures from three independent experiments. One-way ANOVA statistical analysis $(\mathrm{p}<0.05)$ showed no difference between control cells and torin 1 (TOR) treatment or AIgG treatment. Work performed by Dr. Matthew Gray (Gray et al., 2016). 

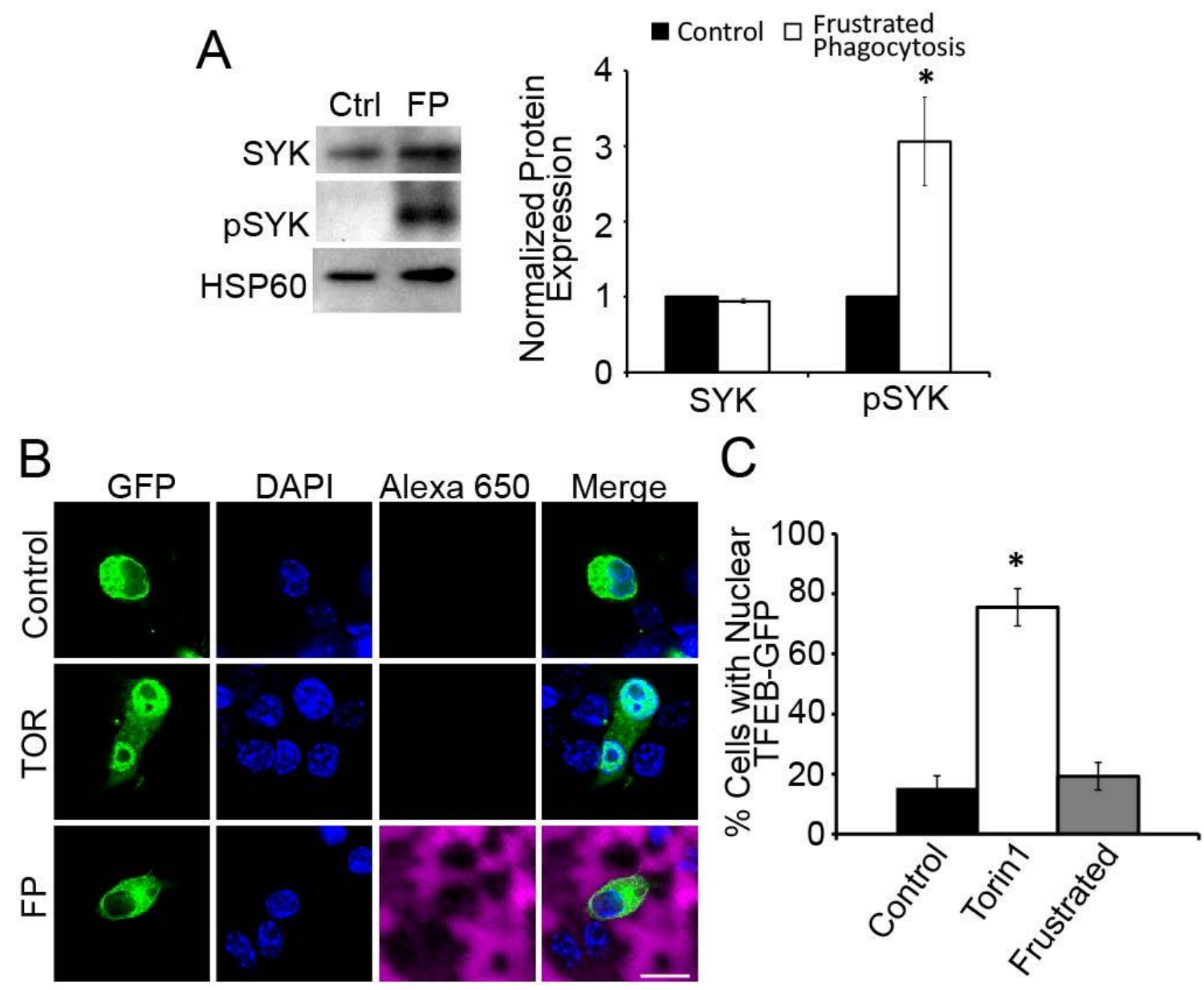
Figure A1.4: Frustrated phagocytosis activates Fcy receptor signaling but does not activate TFEB. Coverslips were coated in BSA and opsonized with anti-BSA IgG. RAW cells were parachuted onto the coverslips. (A) Phosphorylation of Syk as a measure of Fc $\gamma$ receptor activation. Western blot analysis from whole cell lysates following $1 \mathrm{~h}$ incubation on IgGopsonized coverslips compared to unopsonized coverslips. Shown is a representative image from 7 independent experiments. Quantification shown represents that mean abundance \pm SEM of pSyk normalized against total Syk, each normalized against HSP60. (B) RAW cells expressing TFEB-GFP were parachuted onto IgG-opsonized for frustrated phagocytosis (FP) or nonopsonized coverslips. IgG was detected with a secondary antibody. Torin1 was used as a positive control for TFEB nuclear localization. Shown are representative images from three independent experiments. Scale bar represents $10 \mu \mathrm{m}$. (C) Quantification of TFEB-GFP localization from frustrated phagocytosis in (B). Asterisks (*) indicate statistical difference compared to the control condition $(\mathrm{p}<0.05)$ using Student's t-test. Work performed by Dr. Matthew Gray (Gray et al., 2016). 


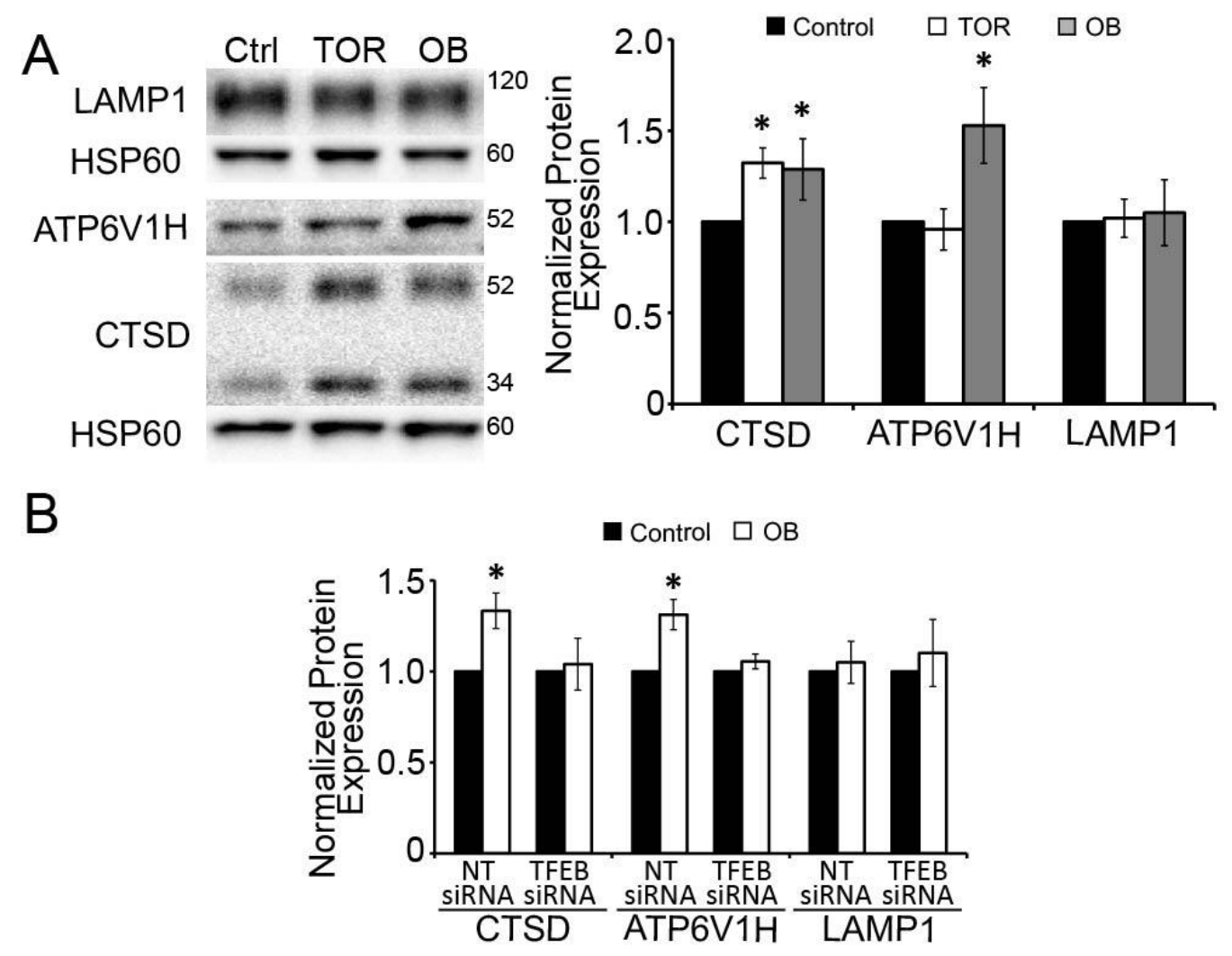


Figure A2.1: Fc $\gamma$ receptor phagocytosis increases protein abundance of some lysosomal proteins. (A) Western blot of whole cell lysates after phagocytosis of IgG-opsonized beads (OB). Protein abundance was compared against torin1 (TOR) treated cells as a positive control. Shown is a representative image from for seven independent experiments for CTSD and ATP6V1H and three independent experiments for LAMP1. Molecular weights are provided to the right of the blot. The accompanying quantification shows mean abundance \pm SEM of the respective proteins normalized against HSP60 abundance. (B) qRT-PCR analysis of whole cell lysates after phagocytosis of IgG-opsonized beads (OB), gene silenced with TFEB or non-targeting (NT) oligonucleotides. The quantification shown represents mean relative mRNA abundance \pm SEM from four independent experiments. Asterisks (*) indicate statistical difference compared to the control condition $(\mathrm{p}<0.05)$ using one-way ANOVA with Tukey's post-hoc test. Work performed by Dr. Matthew Gray (Gray et al., 2016). 


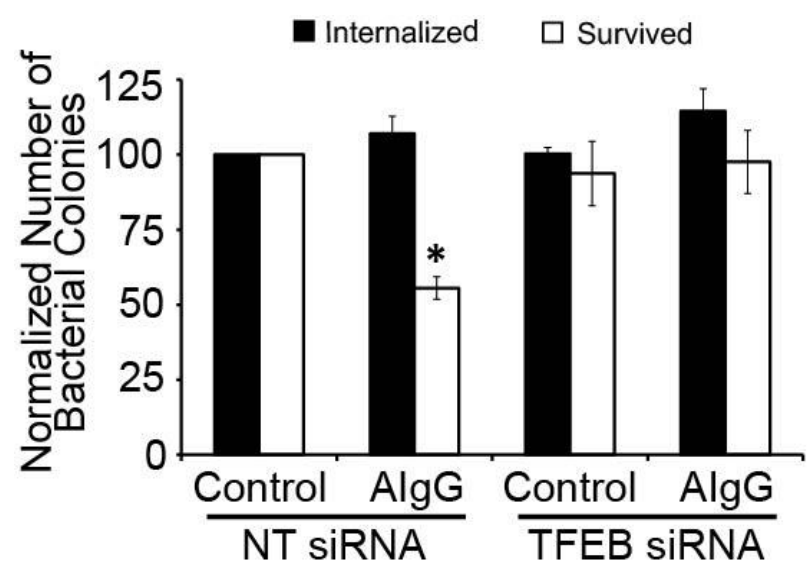


Figure A2.2: Pre-stimulation of the Fc $\gamma$ receptor enhances bacterial killing. RAW cells, gene silenced with TFEB or non-targeting (NT) oligonucleotides, were stimulated with heataggregated $\operatorname{IgG}(\mathrm{AIgG})$ for 3.5 hours. Following initial stimulation, RAW cells were challenged with DH5 $\alpha$ E. coli. RAW cells were either lysed immediately to determine the number of E. coli internalized (black bars) or allowed to degrade the E. coli for 5 hours (white bars). Shown is the normalized mean \pm SEM number of $E$. coli from three independent experiments. Asterisks (*) indicate statistical difference compared to the control condition $(\mathrm{p}<0.05)$ using Student's t-test Work performed by Dr. Matthew Gray (Gray et al., 2016). 


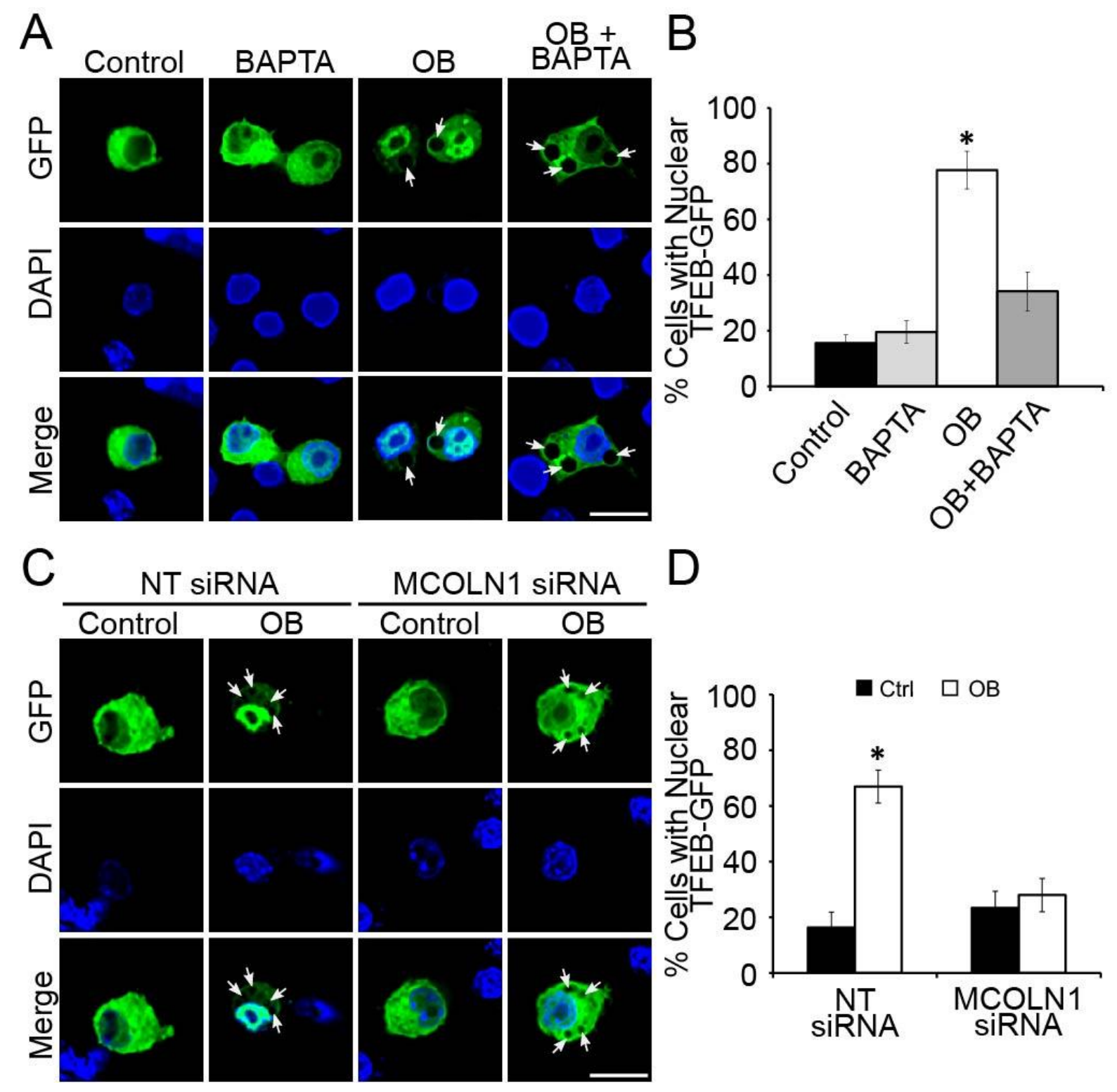


Figure A2.3: TFEB nuclear localization depends on $\mathrm{Ca}^{2+}$ release from mucolipin-1. (A, B) RAW cells expressing TFEB-GFP were challenged with BAPTA-AM prior to phagocytosis of opsonized beads (OB). (A) Representative images are shown. (B) Quantification shown represents mean number of cells \pm SEM with TFEB-GFP in the nucleus from three independent experiments. Asterisks (*) indicate statistical difference compared to the control condition $(\mathrm{p}<0.05)$ using one-way ANOVA followed by Tukey's post-hoc test. $(\mathrm{C}, \mathrm{D})$ RAW cells were silenced with MCOLN1 or non-targeting (NT) siRNA, prior to phagocytosis or OB. (C) Representative images are shown. (D). Quantification shown represents mean number of cells \pm SEM with TFEB-GFP in the nucleus from three independent experiments. Asterisks (*) indicate statistical difference compared to the control condition $(\mathrm{p}<0.05)$ using Student's t-test. DAPI is used as a nuclear counter-stain where indicated. Arrows denote presence of OB. Scale bars represent $10 \mu \mathrm{m}$. Work performed by Dr. Matthew Gray (Gray et al., 2016). 
References 
Abe, N., Inoue, T., Galvez, T., Klein, L., \& Meyer, T. (2008). Dissecting the role of PtdIns(4,5)P2 in endocytosis and recycling of the transferrin receptor. Journal of Cell Science, 121(9), 1488-1494. https://doi.org/10.1242/jcs.020792

Aksan, I., \& Goding, C. R. (1998). Targeting the microphthalmia basic helix-loop-helix-leucine zipper transcription factor to a subset of E-box elements in vitro and in vivo. Molecular and Cellular Biology, 18(12), 6930-8. Retrieved from http://www.ncbi.nlm.nih.gov/pubmed/9819381

Alghamdi, T. A., Ho, C. Y., Mrakovic, A., Taylor, D., Mao, D., \& Botelho, R. J. (2013). Vac14 protein multimerization is a prerequisite step for Fab1 protein complex assembly and function. Journal of Biological Chemistry, 288(13), 9363-9372. https://doi.org/10.1074/jbc.M113.453712

Argani, P., Reuter, V. E., Zhang, L., Sung, Y.-S., Ning, Y., Epstein, J. I., ... Antonescu, C. R. (2016). TFEB-amplified Renal Cell Carcinomas. The American Journal of Surgical Pathology, 40(11), 1484-1495. https://doi.org/10.1097/PAS.0000000000000720

Balla, T. (2013). Phosphoinositides: Tiny Lipids With Giant Impact on Cell Regulation. Physiological Reviews, 93(3), 1019-1137. https://doi.org/10.1152/physrev.00028.2012

Bao, J., Zheng, L., Zhang, Q., Li, X., Zhang, X., Li, Z., ... Ji, J. (2016). Deacetylation of TFEB promotes fibrillar $A \beta$ degradation by upregulating lysosomal biogenesis in microglia. Protein and Cell, 7(6), 417-433. https://doi.org/10.1007/s13238-016-0269-2

Bar-Peled, L., Schweitzer, L. D., Zoncu, R., \& Sabatini, D. M. (2012). Ragulator is a GEF for the rag GTPases that signal amino acid levels to mTORC1. Cell, 150(6), 1196-1208. https://doi.org/10.1016/j.cell.2012.07.032

Bechard, M., \& Dalton, S. (2009). Subcellular Localization of Glycogen Synthase Kinase 3 Controls Embryonic Stem Cell Self-Renewal. Molecular and Cellular Biology, 29(8), 2092-2104. https://doi.org/10.1128/MCB.01405-08

Beckmann, H., Su, L. K., \& Kadesch, T. (1990). TFE3: A helix-loop-helix protein that activates transcription through the immunoglobulin enhancer $\mu \mathrm{E} 3$ motif. Genes and Development, 4(2), 167-179. https://doi.org/10.1101/gad.4.2.167

Bissig, C., Hurbain, I., Raposo, G., \& van Niel, G. (2017). PIKfyve activity regulates reformation of terminal storage lysosomes from endolysosomes. Traffic, 18(11), 747-757. https://doi.org/10.1111/tra.12525

Bo, X., Kim, M., Nori, S. L., Schoepfer, R., Burnstock, G., \& North, R. A. (2003). Tissue distribution of $\mathrm{P} 2 \mathrm{X} 4$ receptors studied with an ectodomain antibody. Cell and Tissue Research, 313(2), 159-165. https://doi.org/10.1007/s00441-003-0758-5

Bohdanowicz, M., \& Grinstein, S. (2013). Role of Phospholipids in Endocytosis, Phagocytosis, and Macropinocytosis. Physiological Reviews, 93(1), 69-106.

https://doi.org/10.1152/physrev.00002.2012

Bone, L. N., Dayam, R. M., Lee, M., Kono, N., Fairn, G. D., Arai, H., ... Antonescu, C. N. (2017). The acyltransferase LYCAT controls specific phosphoinositides and related 
membrane traffic. Molecular Biology of the Cell, 28(1), 161-172.

https://doi.org/10.1091/mbc.E16-09-0668

Botelho, R. J., Efe, J. A., Teis, D., \& Emr, S. D. (2008). Assembly of a Fab1 Phosphoinositide Kinase Signaling Complex Requires the Fig4 Phosphoinositide Phosphatase. Molecular Biology of the Cell, 19(10), 4273-4286. https://doi.org/10.1091/mbc.e08-04-0405

Botelho, R. J., Teruel, M., Dierckman, R., Anderson, R., Wells, A., York, J. D., ... Grinstein, S. (2000). Localized biphasic changes in phosphatidylinositol-4,5-bisphosphate at sites of phagocytosis. Journal of Cell Biology, 151(7), 1353-1367.

https://doi.org/10.1083/jcb.151.7.1353

Bouché, V., Espinosa, A. P., Leone, L., Sardiello, M., Ballabio, A., \& Botas, J. (2016). Drosophila Mitf regulates the V-ATPase and the lysosomal-autophagic pathway. Autophagy, 12(3), 484-498. https://doi.org/10.1080/15548627.2015.1134081

Braulke, T., \& Bonifacino, J. S. (2009). Sorting of lysosomal proteins. Biochimica et Biophysica Acta - Molecular Cell Research, 1793(4), 605-614. https://doi.org/10.1016/j.bbamcr.2008.10.016

Bridges, D., Ma, J.-T., Park, S., Inoki, K., Weisman, L. S., \& Saltiel, A. R. (2012). Phosphatidylinositol 3,5-bisphosphate plays a role in the activation and subcellular localization of mechanistic target of rapamycin 1. Molecular Biology of the Cell, 23(15), 2955-2962. https://doi.org/10.1091/mbc.E11-12-1034

Bright, N. A., Davis, L. J., \& Luzio, J. P. (2016). Endolysosomes Are the Principal Intracellular Sites of Acid Hydrolase Activity. Current Biology : CB, 26(17), 2233-45. https://doi.org/10.1016/j.cub.2016.06.046

Brown, E. J., Beal, P. A., Keith, C. T., Chen, J., Shin, T. B., \& Schreiber, S. L. (1995). Control of p70 S6 kinase by kinase activity of FRAP in vivo. Nature, 377(6548), 441-446. https://doi.org/10.1038/377441a0

Brunn, G. J., Hudson, C. C., Sekulić, A., Williams, J. M., Hosoi, H., Houghton, P. J., ... Abraham, R. T. (1997). Phosphorylation of the translational repressor PHAS-I by the mammalian target of rapamycin. Science, 277(5322), 99-101. https://doi.org/10.1126/science.277.5322.99

Bryant, N. J., Piper, R. C., Weisman, L. S., \& Stevens, T. H. (1998). Retrograde traffic out of the yeast vacuole to the TGN occurs via the prevacuolar/endosomal compartment. Journal of Cell Biology, 142(3), 651-663. https://doi.org/10.1083/jcb.142.3.651

Burd, C. G., \& Emr, S. D. (1998). Phosphatidylinositol(3)-phosphate signaling mediated by specific binding to RING FYVE domains. Molecular Cell, 2(1), 157-162. https://doi.org/10.1016/S1097-2765(00)80125-2

Cabrera, M., Nordmann, M., Perz, A., Schmedt, D., Gerondopoulos, A., Barr, F., ... Ungermann, C. (2014). The Mon1-Ccz1 GEF activates the Rab7 GTPase Ypt7 via a longin-fold-Rab interface and association with PI3P-positive membranes. Journal of Cell Science, 127(5), 1043-1051. https://doi.org/10.1242/jcs.140921 
Cai, X., Xu, Y., Cheung, A. K., Tomlinson, R. C., Alcázar-Román, A., Murphy, L., ... Huang, Q. (2013). PIKfyve, a class III PI Kinase, is the target of the small molecular IL-12/IL-23 inhibitor apilimod and a player in toll-like receptor signaling. Chemistry and Biology, 20(7), 912-921. https://doi.org/10.1016/j.chembiol.2013.05.010

Campbell, G. R., Rawat, P., Bruckman, R. S., \& Spector, S. A. (2015). Human Immunodeficiency Virus Type 1 Nef Inhibits Autophagy through Transcription Factor EB Sequestration. PLoS Pathogens, 11(6), e1005018. https://doi.org/10.1371/journal.ppat.1005018

Canfield, S. M. (1991). The binding affinity of human IgG for its high affinity Fc receptor is determined by multiple amino acids in the $\mathrm{CH} 2$ domain and is modulated by the hinge region. Journal of Experimental Medicine, 173(6), 1483-1491. https://doi.org/10.1084/jem.173.6.1483

Carlton, J., Bujny, M., Peter, B. J., Oorschot, V. M. J., Rutherford, A., Mellor, H., ... Cullen, P. J. (2004). Sorting nexin-1 mediates tubular endosome-to-TGN transport through coincidence sensing of high- curvature membranes and 3-phosphoinositides. Current Biology, 14(20), 1791-1800. https://doi.org/10.1016/j.cub.2004.09.077

Chantranupong, L., Scaria, S. M., Saxton, R. A., Gygi, M. P., Shen, K., Wyant, G. A., ... Sabatini, D. M. (2016). The CASTOR Proteins Are Arginine Sensors for the mTORC1 Pathway. Cell, 165(1), 153-164. https://doi.org/10.1016/j.cell.2016.02.035

Chauhan, S., Goodwin, J. G., Chauhan, S., Manyam, G., Wang, J., Kamat, A. M., \& Boyd, D. D. (2013). ZKSCAN3 Is a Master Transcriptional Repressor of Autophagy. Molecular Cell, 50(1), 16-28. https://doi.org/10.1016/j.molcel.2013.01.024

Chen, L., Wang, K., Long, A., Jia, L., Zhang, Y., Deng, H., ... Wang, Y. (2017). Fastinginduced hormonal regulation of lysosomal function. Cell Research, 27(6), 748-763. https://doi.org/10.1038/cr.2017.45

Choy, C. H., Han, B. K., \& Botelho, R. J. (2017). Phosphoinositide Diversity, Distribution, and Effector Function: Stepping Out of the Box. BioEssays, 39(12), 1700121. https://doi.org/10.1002/bies.201700121

Choy, C. H., Saffi, G., Gray, M. A., Wallace, C., Dayam, R. M., Ou, Z.-Y. A., .. Botelho, R. J. (2018). Lysosome enlargement during inhibition of the lipid kinase PIKfyve proceeds through lysosome coalescence. Journal of Cell Science, 131(10), jcs213587. https://doi.org/10.1242/jcs.213587

Christensen, K. a, Myers, J. T., \& Swanson, J. a. (2002). pH-dependent regulation of lysosomal calcium in macrophages. Journal of Cell Science, 115(Pt 3), 599-607. https://doi.org/10.1073/pnas.89.21.10079

Christoforidis, S., McBride, H. M., Burgoyne, R. D., \& Zerial, M. (1999). The rab5 effector EEA1 is a core component of endosome docking. Nature, 397(6720), 621-625. https://doi.org/10.1038/17618

Clemens, D. L., Lee, B. Y., \& Horwitz, M. A. (2002). The Mycobacterium tuberculosis 
phagosome in human macrophages is isolated from the host cell cytoplasm. Infection and Immunity, 70(10), 5800-5807. https://doi.org/10.1128/IAI.70.10.5800-5807.2002

Cole, A., Frame, S., \& Cohen, P. (2004). Further evidence that the tyrosine phosphorylation of glycogen synthase kinase-3 (GSK3) in mammalian cells is an autophosphorylation event. Biochemical Journal, 377(1), 249-255. https://doi.org/10.1042/bj20031259

Collins, R. F., Schreiber, A. D., Grinstein, S., \& Trimble, W. S. (2002). Syntaxins 13 and 7 Function at Distinct Steps During Phagocytosis. The Journal of Immunology, 169(6), 32503256. https://doi.org/10.4049/jimmunol.169.6.3250

Cosson, P., \& Soldati, T. (2008). Eat, kill or die: when amoeba meets bacteria. Current Opinion in Microbiology, 11(3), 271-276. https://doi.org/10.1016/j.mib.2008.05.005

Cowland, J. B., \& Borregaard, N. (2016). Granulopoiesis and granules of human neutrophils. Immunological Reviews, 273(1), 11-28. https://doi.org/10.1111/imr.12440

Cox, D., Berg, J. S., Cammer, M., Chinegwundoh, J. O., Dale, B. M., Cheney, R. E., \& Greenberg, S. (2002). Myosin X is a downstream effector of PI(3)K during phagocytosis. Nature Cell Biology, 4(7), 469-477. https://doi.org/10.1038/ncb805

Cox, D., Dale, B. M., Kashiwada, M., Helgason, C. D., \& Greenberg, S. (2001). A regulatory role for Src homology 2 domain-containing inositol 5'-phosphatase (SHIP) in phagocytosis mediated by Fc gamma receptors and complement receptor 3 (alpha(M)beta(2); CD11b/CD18). The Journal of Experimental Medicine, 193(1), 61-71. https://doi.org/10.1084/jem.193.1.61

Craige, B., Salazar, G., \& Faundez, V. (2008). Phosphatidylinositol-4-Kinase Type II Alpha Contains an AP-3-sorting Motif and a Kinase Domain That Are Both Required for Endosome Traffic. Molecular Biology of the Cell, 19(4), 1415-1426. https://doi.org/10.1091/mbc.E07-12-1239

Cross, D. A. E., Alessi, D. R., Cohen, P., Andjelkovich, M., \& Hemmings, B. A. (1995). Inhibition of glycogen synthase kinase- 3 by insulin mediated by protein kinase B. Nature, 378(6559), 785-789. https://doi.org/10.1038/378785a0

Dart, A. E., Donnelly, S. K., Holden, D. W., Way, M., \& Caron, E. (2012). Nck and Cdc42 cooperate to recruit N-WASP to promote Fc $\gamma \mathrm{R}$-mediated phagocytosis. Journal of Cell Science, 125(12), 2825-2830. https://doi.org/10.1242/jcs.106583

Dayam, R. M., Saric, A., Shilliday, R. E., \& Botelho, R. J. (2015). The Phosphoinositide-Gated Lysosomal $\mathrm{Ca}^{2+}$ Channel, TRPML1, Is Required for Phagosome Maturation. Traffic, 16(9), 1010-1026. https://doi.org/10.1111/tra.12303

de Lartigue, J., Polson, H., Feldman, M., Shokat, K., Tooze, S. A., Urbé, S., \& Clague, M. J. (2009). PIKfyve regulation of endosome-linked pathways. Traffic, 10(7), 883-893. https://doi.org/10.1111/j.1600-0854.2009.00915.x

Deckert, M., Tartare-Deckert, S., Couture, C., Mustelin, T., \& Altman, A. (1996). Functional and physical interactions of Syk family kinases with the Vav proto-oncogene product. Immunity, 5(6), 591-604. https://doi.org/10.1016/S1074-7613(00)80273-3 
Decressac, M., Mattsson, B., Weikop, P., Lundblad, M., Jakobsson, J., \& Bjorklund, A. (2013). TFEB-mediated autophagy rescues midbrain dopamine neurons from -synuclein toxicity. Proceedings of the National Academy of Sciences, 110(19), E1817-E1826. https://doi.org/10.1073/pnas.1305623110

Dietrich, J., Kastrup, J., Nielsen, B. L., Ødum, N., \& Geisler, C. (1997). Regulation and function of the CD3 $\gamma /$ DxxxLL motif: A binding site for adaptor protein-1 and adaptor protein-2 in vitro. Journal of Cell Biology, 138(2), 271-281. https://doi.org/10.1083/jcb.138.2.271

Divecha, N., Roefs, M., Halstead, J. R., D’Andrea, S., Fernandez-Borga, M., Oomen, L., ... D'Santos, C. (2000). Interaction of the type Ialpha PIPkinase with phospholipase D: a role for the local generation of phosphatidylinositol 4, 5-bisphosphate in the regulation of PLD2 activity. The EMBO Journal, 19(20), 5440-9. https://doi.org/10.1093/emboj/19.20.5440

Dong, X., Shen, D., Wang, X., Dawson, T., Li, X., Zhang, Q., ... Xu, H. (2010). PI(3,5)P2 controls membrane trafficking by direct activation of mucolipin $\mathrm{Ca} 2+$ release channels in the endolysosome. Nature Communications, 1(4), 1-11. https://doi.org/10.1038/ncomms 1037

Dove, S. K., Cooke, F. T., Douglas, M. R., Sayers, L. G., Parker, P. J., \& Michell, R. H. (1997). Osmotic stress activates phosphatidylinositol-3,5-bisphosphate synthesis. Nature, 390(6656), 187-192. https://doi.org/10.1038/36613

Dove, S. K., McEwen, R. K., Mayes, A., Hughes, D. C., Beggs, J. D., \& Michell, R. H. (2002). Vac14 controls PtdIns(3,5)P2synthesis and Fab1-dependent protein trafficking to the multivesicular body. Current Biology, 12(11), 885-893. https://doi.org/10.1016/S09609822(02)00891-6

Dove, S. K., Piper, R. C., McEwen, R. K., Yu, J. W., King, N. C., Hughes, D. C., ... Lemmon, M. A. (2004). Svp1p defines a family of phosphatidylinositol 3,5-bisphosphate effectors. EMBO Journal, 23(9), 1922-1933. https://doi.org/10.1038/sj.emboj.7600203

Dransart, E., Morin, A., Cherfils, J., \& Olofsson, B. (2005). Uncoupling of inhibitory and shuttling functions of Rho GDP dissociation inhibitors. Journal of Biological Chemistry, 280(6), 4674-4683. https://doi.org/10.1074/jbc.M409741200

Efe, J. A., Botelho, R. J., \& Emr, S. D. (2005). The Fab1 phosphatidylinositol kinase pathway in the regulation of vacuole morphology. Current Opinion in Cell Biology, 17(4), 402-408. https://doi.org/10.1016/j.ceb.2005.06.002

Escobar, E. O. (2017). The role of phagocytosis-dependent activation of TFEB in the clearance of salmonella. Ryerson University.

Fairn, G. D., Ogata, K., Botelho, R. J., Stahl, P. D., Anderson, R. A., De Camilli, P., ... Grinstein, S. (2009). An electrostatic switch displaces phosphatidylinositol phosphate kinases from the membrane during phagocytosis. Journal of Cell Biology, 187(5), 701-714. https://doi.org/10.1083/jcb.200909025

Ferguson, C. J., Lenk, G. M., Jones, J. M., Grant, A. E., Winters, J. J., Dowling, J. J., ... Meisler, M. H. (2012). Neuronal expression of Fig4 is both necessary and sufficient to prevent 
spongiform neurodegeneration. Human Molecular Genetics, 21(16), 3525-3534. https://doi.org/10.1093/hmg/dds179

Ferguson, C. J., Lenk, G. M., \& Meisler, M. H. (2009). Defective autophagy in neurons and astrocytes from mice deficient in PI(3,5)P2. Human Molecular Genetics, 18(24), 48684878. https://doi.org/10.1093/hmg/ddp460

Ferron, M., Settembre, C., Shimazu, J., Lacombe, J., Kato, S., Rawlings, D. J., ... Karsenty, G. (2013). A RANKL-PKC $\beta$-TFEB signaling cascade is necessary for lysosomal biogenesis in osteoclasts. Genes and Development, 27(8), 955-969. https://doi.org/10.1101/gad.213827.113

Flannagan, R. S., Harrison, R. E., Yip, C. M., Jaqaman, K., \& Grinstein, S. (2010). Dynamic macrophage "probing" is required for the efficient capture of phagocytic targets. Journal of Cell Biology, 191(6), 1205-1218. https://doi.org/10.1083/jcb.201007056

Flannagan, R. S., Jaumouillé, V., \& Grinstein, S. (2012). The Cell Biology of Phagocytosis. Annual Review of Pathology: Mechanisms of Disease, 7(1), 61-98. https://doi.org/10.1146/annurev-pathol-011811-132445

Frame, S., Cohen, P., \& Biondi, R. M. (2001). A common phosphate binding site explains the unique substrate specificity of GSK3 and its inactivation by phosphorylation. Molecular Cell, 7(6), 1321-1327. https://doi.org/10.1016/S1097-2765(01)00253-2

Freeman, S. A., Goyette, J., Furuya, W., Woods, E. C., Bertozzi, C. R., Bergmeier, W., ... Grinstein, S. (2016). Integrins Form an Expanding Diffusional Barrier that Coordinates Phagocytosis. Cell, 164(1-2), 128-140. https://doi.org/10.1016/j.cell.2015.11.048

Garami, A., Zwartkruis, F. J. T., Nobukuni, T., Joaquin, M., Roccio, M., Stocker, H., ... Thomas, G. (2003). Insulin activation of Rheb, a mediator of mTOR/S6K/4E-BP signaling, is inhibited by TSC1 and 2. Molecular Cell, 11(6), 1457-1466. https://doi.org/10.1016/S10972765(03)00220-X

Garc1, E., \& Rosales, C. (2001). Phosphatidylinositol 3-kinase and ERK are required for NF- B activation but not for phagocytosis Abstract: The molecular events that transduce sig- nals from Fc receptors to the various cellular responses are still poorly defined. We have investigated. Journal of Leukocyte Biology, 70(4), 649-58. https://doi.org/10.1189/jlb.70.4.649

Garred, P., Michaelsen, T. E., \& Aase, A. (1989). The IgG Subclass Pattern of Complement Activation Depends on Epitope Density and Antibody and Complement Concentration. Scandinavian Journal of Immunology, 30(3), 379-382. https://doi.org/10.1111/j.13653083.1989.tb01225.x

Gary, J. D., Sato, T. K., Stefan, C. J., Bonangelino, C. J., Weisman, L. S., \& Emr, S. D. (2002). Regulation of Fab1 Phosphatidylinositol 3-Phosphate 5-Kinase Pathway by Vac7 Protein and Fig4, a Polyphosphoinositide Phosphatase Family Member. Molecular Biology of the Cell, 13(4), 1238-1251. https://doi.org/10.1091/mbc.01-10-0498

Gary, J. D., Wurmser, A. E., Bonangelino, C. J., Weisman, L. S., \& Emr, S. D. (1998). Fab1p is 
essential for PtdIns(3)P 5-kinase activity and the maintenance of vacuolar size and membrane homeostasis. Journal of Cell Biology, 143(1), 65-79.

https://doi.org/10.1083/jcb.143.1.65

Gayle, S., Landrette, S., Beeharry, N., Conrad, C., Hernandez, M., Beckett, P., ... Lichenstein, H. (2017). Identification of apilimod as a first-in-class PIKfyve kinase inhibitor for treatment of B-cell non-Hodgkin lymphoma. Blood, 129(13), 1768-1778. https://doi.org/10.1182/blood-2016-09-736892

Ghazizadeh, S., Bolen, J. B., \& Fleit, H. B. (1994). Physical and functional association of Srcrelated protein tyrosine kinases with Fc??RII in monocytic THP-1 cells. Journal of Biological Chemistry, 269(12), 8878-8884. Retrieved from http://www.ncbi.nlm.nih.gov/pubmed/8132624

Giaccia, A. J., \& Kastan, M. B. (1998). The complexity of p53 modulation: Emerging patterns from divergent signals. Genes and Development, 12(19), 2973-2983. https://doi.org/10.1101/gad.12.19.2973

Goc, A., Al-Husein, B. A.-H., Katsanevas, K., Steinbach, A., Lou, U., Sabbineni, H., ... Somanath, P. R. (2014). Targeting Src-mediated Tyr216 phosphorylation and activation of GSK-3 in prostate cancer cells inhibit prostate cancer progression \&lt;i\&gt;in vitro\&lt;/i\&gt; and \&lt;i\&gt;in vivo\&lt;/i\&gt; Oncotarget, 5(3), 775-787. https://doi.org/10.18632/oncotarget.1770

Gopaldass, N., Fauvet, B., Lashuel, H., Roux, A., \& Mayer, A. (2017). Membrane scission driven by the PROPPIN Atg18. The EMBO Journal, 36(22), e201796859. https://doi.org/10.15252/embj.201796859

Gore, S., Andersson, J., Biran, R., Underwood, C., \& Riesenfeld, J. (2014). Heparin surfaces: Impact of immobilization chemistry on hemocompatibility and protein adsorption. Journal of Biomedical Materials Research - Part B Applied Biomaterials, 102(8), 1817-1824. https://doi.org/10.1002/jbm.b.33154

Gray, M. A., Choy, C. H., Dayam, R. M., Ospina-Escobar, E., Somerville, A., Xiao, X., ... Botelho, R. J. (2016). Phagocytosis Enhances Lysosomal and Bactericidal Properties by Activating the Transcription Factor TFEB. Current Biology, 26(15), 1955-1964. https://doi.org/10.1016/j.cub.2016.05.070

Gu, H., Botelho, R. J., Yu, M., Grinstein, S., \& Neel, B. G. (2003). Critical role for scaffolding adapter Gab2 in FcyR-mediated phagocytosis. Journal of Cell Biology, 161(6), 1151-1161. https://doi.org/10.1083/jcb.200212158

Hall, A. B., Gakidis, M. A. M., Glogauer, M., Wilsbacher, J. L., Gao, S., Swat, W., \& Brugge, J. S. (2006). Requirements for Vav guanine nucleotide exchange factors and Rho GTPases in FcyR- and complement-mediated phagocytosis. Immunity, 24(3), 305-316. https://doi.org/10.1016/j.immuni.2006.02.005

Hamada, F., Aoki, M., Akiyama, T., \& Toyoshima, K. (1993). Association of immunoglobulin G Fc receptor II with Src-like protein-tyrosine kinase Fgr in neutrophils. Proceedings of the National Academy of Sciences, 90(13), 6305-6309. https://doi.org/10.1073/pnas.90.13.6305 
Hammond, G. R. V., \& Balla, T. (2015). Polyphosphoinositide binding domains: Key to inositol lipid biology. Biochimica et Biophysica Acta - Molecular and Cell Biology of Lipids, 1851(6), 746-758. https://doi.org/10.1016/j.bbalip.2015.02.013

Han, B. K., \& Emr, S. D. (2011). Phosphoinositide [PI(3,5)P2] lipiddependent regulation of the general transcriptional regulator Tup1. Genes and Development, 25(9), 984-995. https://doi.org/10.1101/gad.1998611

Harrison, R. E., Bucci, C., Vieira, O. V., Schroer, T. A., \& Grinstein, S. (2003). Phagosomes Fuse with Late Endosomes and/or Lysosomes by Extension of Membrane Protrusions along Microtubules: Role of Rab7 and RILP. Molecular and Cellular Biology, 23(18), 64946506. https://doi.org/10.1128/MCB.23.18.6494-6506.2003

Hemesath, T. J., Steingrimsson, E., McGill, G., Hansen, M. J., Vaught, J., Hodgkinson, C. A., ... Fisher, D. E. (1994). microphthalmia, a critical factor in melanocyte development, defines a discrete transcription factor family. Genes \& Development, 8(22), 2770-2780. https://doi.org/10.1101/gad.8.22.2770

Henne, W. M., Buchkovich, N. J., \& Emr, S. D. (2011). The ESCRT Pathway. Developmental Cell, 21(1), 77-91. https://doi.org/10.1016/j.devcel.2011.05.015

Hicks, A. M., DeLong, C. J., Thomas, M. J., Samuel, M., \& Cui, Z. (2006). Unique molecular signatures of glycerophospholipid species in different rat tissues analyzed by tandem mass spectrometry. Biochimica et Biophysica Acta - Molecular and Cell Biology of Lipids, 1761(9), 1022-1029. https://doi.org/10.1016/j.bbalip.2006.05.010

Higgs, H. N., \& Pollard, T. D. (2000). Activation by Cdc42 and PIP2of Wiskott-Aldrich Syndrome protein (WASp) stimulates actin nucleation by Arp2/3 complex. Journal of Cell Biology, 150(6), 1311-1320. https://doi.org/10.1083/jcb.150.6.1311

Ho, C. Y., Choy, C. H., Wattson, C. A., Johnson, D. E., \& Botelho, R. J. (2015). The Fab1/PIKfyve phosphoinositide phosphate kinase is not necessary to maintain the $\mathrm{pH}$ of lysosomes and of the yeast vacuole. Journal of Biological Chemistry, 290(15), 9919-9928. https://doi.org/10.1074/jbc.M114.613984

Holowka, D., Sil, D., Torigoe, C., \& Baird, B. (2007). Insights into immunoglobulin E receptor signaling from structurally defined ligands. Immunological Reviews, 217(1), 269-279. https://doi.org/10.1111/j.1600-065X.2007.00517.x

Hoppe, A. D. (2004). Cdc42, Rac1, and Rac2 Display Distinct Patterns of Activation during Phagocytosis. Molecular Biology of the Cell, 15(8), 3509-3519. https://doi.org/10.1091/mbc.E03-11-0847

Horazdovsky, B. F., Davies, B. A., Seaman, M. N., McLaughlin, S. A., Yoon, S., \& Emr, S. D. (1997). A sorting nexin-1 homologue, Vps5p, forms a complex with Vps17p and is required for recycling the vacuolar protein-sorting receptor. Molecular Biology of the Cell, 8(8), 1529-41. https://doi.org/10.1091/mbc.8.8.1529

Hosokawa, N., Hara, T., Kaizuka, T., Kishi, C., Takamura, A., Miura, Y., ... Mizushima, N. (2009). Nutrient-dependent mTORC1 Association with the ULK1-Atg13-FIP200 Complex 
Required for Autophagy. Molecular Biology of the Cell, 20(7), 1981-1991. https://doi.org/10.1091/mbc.E08-12-1248

Hsu, C. L., Lee, E. X., Gordon, K. L., Paz, E. A., Shen, W. C., Ohnishi, K., ... La Spada, A. R. (2018). MAP4K3 mediates amino acid-dependent regulation of autophagy via phosphorylation of TFEB. Nature Communications, 9(1), 942. https://doi.org/10.1038/s41467-018-03340-7

Hughes, K., Nikolakaki, E., Plyte, S. E., Totty, N. F., \& Woodgett, J. R. (1993). Modulation of the glycogen synthase kinase-3 family by tyrosine phosphorylation. The EMBO Journal, 12(2), 803-8. https://doi.org/10.1002/j.1460-2075.1993.tb05715.x

Humphries, A. C., Donnelly, S. K., \& Way, M. (2014). Cdc42 and the Rho GEF intersectin-1 collaborate with Nck to promote N-WASP-dependent actin polymerisation. Journal of Cell Science, 127(3), 673-685. https://doi.org/10.1242/jcs.141366

Ibarrola, I., Vossebeld, P. J. M., Homburg, C. H. E., Thelen, M., Roos, D., \& Verhoeven, A. J. (1997). Influence of tyrosine phosphorylation on protein interaction with Fc $\gamma$ RIIa. Biochimica et Biophysica Acta - Molecular Cell Research, 1357(3), 348-358. https://doi.org/10.1016/S0167-4889(97)00034-7

Ikonomov, O. C., Sbrissa, D., Delvecchio, K., Xie, Y., Jin, J. P., Rappolee, D., \& Shisheva, A. (2011). The phosphoinositide kinase PIKfyve is vital in early embryonic development: Preimplantation lethality of PIKfyve-/- embryos but normality of PIKfyve+/- mice. Journal of Biological Chemistry, 286(15), 13404-13413. https://doi.org/10.1074/jbc.M111.222364

Ikonomov, O. C., Sbrissa, D., Mlak, K., Deeb, R., Fligger, J., Soans, A., ... Shisheva, A. (2003). Active PIKfyve Associates with and Promotes the Membrane Attachment of the Late Endosome-to-trans-Golgi Network Transport Factor Rab9 Effector p40. Journal of Biological Chemistry, 278(51), 50863-50871. https://doi.org/10.1074/jbc.M307260200

Ikonomov, O. C., Sbrissa, D., \& Shisheva, A. (2001). Mammalian Cell Morphology and Endocytic Membrane Homeostasis Require Enzymatically Active Phosphoinositide 5Kinase PIKfyve. Journal of Biological Chemistry, 276(28), 26141-26147. https://doi.org/10.1074/jbc.M101722200

Ikonomov, O. C., Sbrissa, D., Venkatareddy, M., Tisdale, E., Garg, P., \& Shisheva, A. (2015). Class III PI 3-kinase is the main source of PtdIns3P substrate and membrane recruitment signal for PIKfyve constitutive function in podocyte endomembrane homeostasis. Biochimica et Biophysica Acta - Molecular Cell Research, 1853(5), 1240-1250. https://doi.org/10.1016/j.bbamcr.2015.01.008

Ivankovic, D., Chau, K. Y., Schapira, A. H. V., \& Gegg, M. E. (2016). Mitochondrial and lysosomal biogenesis are activated following PINK1/parkin-mediated mitophagy. Journal of Neurochemistry, 136(2), 388-402. https://doi.org/10.1111/jnc.13412

Jefferies, H. B. J., Cooke, F. T., Jat, P., Boucheron, C., Koizumi, T., Hayakawa, M., ... Parker, P. J. (2008). A selective PIKfyve inhibitor blocks PtdIns(3,5)P2 production and disrupts endomembrane transport and retroviral budding. EMBO Reports, 9(2), 164-170. https://doi.org/10.1038/sj.embor.7401155 
Jin, N., Chow, C. Y., Liu, L., Zolov, S. N., Bronson, R., Davisson, M., ... Weisman, L. S. (2008). VAC14 nucleates a protein complex essential for the acute interconversion of PI3P and PI(3,5)P2in yeast and mouse. EMBO Journal, 27(24), 3221-3234. https://doi.org/10.1038/emboj.2008.248

Jin, N., Mao, K., Jin, Y., Tevzadze, G., Kauffman, E. J., Park, S., ... Weisman, L. S. (2014). Roles for PI(3,5)P2 in nutrient sensing through TORC1. Molecular Biology of the Cell, 25(7), 1171-1185. https://doi.org/10.1091/mbc.E14-01-0021

Johansson, M., Rocha, N., Zwart, W., Jordens, I., Janssen, L., Kuijl, C., ... Neefjes, J. (2007). Activation of endosomal dynein motors by stepwise assembly of Rab7-RILP-p150Glued, ORP1L, and the receptor BIII spectrin. Journal of Cell Biology, 176(4), 459-471. https://doi.org/10.1083/jcb.200606077

Johnson, S. A., Pleiman, C. M., Pao, L., Schneringer, J., Hippen, K., \& Cambier, J. C. (1995). Phosphorylated immunoreceptor signaling motifs (ITAMs) exhibit unique abilities to bind and activate Lyn and Syk tyrosine kinases. The Journal of Immunology, 155(10), 45964603. Retrieved from http://www.ncbi.nlm.nih.gov/pubmed/7594458

Joshi, S., Singh, A. R., Zulcic, M., \& Durden, D. L. (2014). A PKC-SHP1 signaling axis desensitizes Fc $\gamma$ receptor signaling by reducing the tyrosine phosphorylation of CBL and regulates Fc $\gamma \mathrm{R}$ mediated phagocytosis. BMC Immunology, 15(1), 18. https://doi.org/10.1186/1471-2172-15-18

Jovic, M., Kean, M. J., Szentpetery, Z., Polevoy, G., Gingras, A.-C., Brill, J. A., \& Balla, T. (2012). Two phosphatidylinositol 4-kinases control lysosomal delivery of the Gaucher disease enzyme, -glucocerebrosidase. Molecular Biology of the Cell, 23(8), 1533-1545. https://doi.org/10.1091/mbc.E11-06-0553

Kabeya, Y. (2000). LC3, a mammalian homologue of yeast Apg8p, is localized in autophagosome membranes after processing. The EMBO Journal, 19(21), 5720-5728. https://doi.org/10.1093/emboj/19.21.5720

Kang, S. A., Pacold, M. E., Cervantes, C. L., Lim, D., Lou, H. J., Ottina, K., ... Sabatini, D. M. (2013). mTORC1 phosphorylation sites encode their sensitivity to starvation and rapamycin. Science, 341(6144), 1236566. https://doi.org/10.1126/science.1236566

Katzmann, D. J., Babst, M., \& Emr, S. D. (2001). Ubiquitin-dependent sorting into the multivesicular body pathway requires the function of a conserved endosomal protein sorting complex, ESCRT-I. Cell, 106(2), 145-155. https://doi.org/10.1016/S0092-8674(01)00434-2

Kim, A. S., Kakalis, L. T., Abdul-Manan, N., Liu, G. A., \& Rosen, M. K. (2000). Autoinhibition and activation mechanisms of the Wiskott-Aldrich syndrome protein. Nature, 404(6774), 151-158. https://doi.org/10.1038/35004513

Kim, G. H. E., Dayam, R. M., Prashar, A., Terebiznik, M., \& Botelho, R. J. (2014). PIKfyve inhibition interferes with phagosome and endosome maturation in macrophages. Traffic, 15(10), 1143-1163. https://doi.org/10.1111/tra.12199

Kim, S. M., Roy, S. G., Chen, B., Nguyen, T. M., McMonigle, R. J., McCracken, A. N., ... 
Edinger, A. L. (2016). Targeting cancer metabolism by simultaneously disrupting parallel nutrient access pathways. Journal of Clinical Investigation, 126(11), 4088-4102. https://doi.org/10.1172/JCI87148

Kinchen, J. M., \& Ravichandran, K. S. (2010). Identification of two evolutionarily conserved genes regulating processing of engulfed apoptotic cells. Nature, 464(7289), 778-782. https://doi.org/10.1038/nature08853

Kinoshita, E., Kinoshita-Kikuta, E., Takiyama, K., \& Koike, T. (2006). Phosphate-binding Tag, a New Tool to Visualize Phosphorylated Proteins. Molecular \& Cellular Proteomics, 5(4), 749-757. https://doi.org/10.1074/mcp.T500024-MCP200

Korolchuk, V. I., Saiki, S., Lichtenberg, M., Siddiqi, F. H., Roberts, E. A., Imarisio, S., ... Rubinsztein, D. C. (2011). Lysosomal positioning coordinates cellular nutrient responses. Nature Cell Biology, 13(4), 453-462. https://doi.org/10.1038/ncb2204

Krishna, S., Palm, W., Lee, Y., Yang, W., Bandyopadhyay, U., Xu, H., ... Overholtzer, M. (2016). PIKfyve Regulates Vacuole Maturation and Nutrient Recovery following Engulfment. Developmental Cell, 38(5), 536-547. https://doi.org/10.1016/j.devcel.2016.08.001

Kuiper, R. P., Schepens, M., Thijssen, J., van Asseldonk, M., van den Berg, E., Bridge, J., ... van Kessel, A. G. (2003). Upregulation of the transcription factor TFEB in $\mathrm{t}(6 ; 11)(\mathrm{p} 21 ; \mathrm{q} 13)-$ positive renal cell carcinomas due to promoter substitution. Human Molecular Genetics, 12(14), 1661-1669. https://doi.org/10.1093/hmg/ddg178

Kusner, D. J., Hall, C. F., \& Jackson, S. (1999). Fc gamma receptor-mediated activation of phospholipase D regulates macrophage phagocytosis of IgG-opsonized particles. The Journal of Immunology, 162(4), 2266-2274. Retrieved from http://www.ncbi.nlm.nih.gov/pubmed/9973503

Larsen, E. C., Ueyama, T., Brannock, P. M., Shirai, Y., Saito, N., Larsson, C., ... Lennartz, M. R. (2002). A role for PKC- $\varepsilon$ in Fc $\gamma$ R-mediated phagocytosis by RAW 264.7 cells. Journal of Cell Biology, 159(6), 939-944. https://doi.org/10.1083/jcb.200205140

Lawe, D. C., Chawla, A., Merithew, E., Dumas, J., Carrington, W., Fogarty, K., ... Corvera, S. (2002). Sequential roles for phosphatidylinositol 3-phosphate and Rab5 in tethering and fusion of early endosomes via their interaction with EEA1. Journal of Biological Chemistry, 277(10), 8611-8617. https://doi.org/10.1074/jbc.M109239200

Lee, W. L., Cosio, G., Ireton, K., \& Grinstein, S. (2007). Role of CrkII in Fc?? receptormediated phagocytosis. Journal of Biological Chemistry, 282(15), 11135-11143. https://doi.org/10.1074/jbc.M700823200

Lenk, G. M., Frei, C. M., Miller, A. C., Wallen, R. C., Mironova, Y. A., Giger, R. J., \& Meisler, M. H. (2016). Rescue of neurodegeneration in the Fig4 null mouse by a catalytically inactive FIG4 transgene. Human Molecular Genetics, 25(2), 340-347. https://doi.org/10.1093/hmg/ddv480

Li, L., Friedrichsen, H. J., Andrews, S., Picaud, S., Volpon, L., Ngeow, K., ... Goding, C. R. 
(2018). A TFEB nuclear export signal integrates amino acid supply and glucose availability. Nature Communications, 9(1), 2685. https://doi.org/10.1038/s41467-018-04849-7

Li, S. C., Diakov, T. T., Xu, T., Tarsio, M., Zhu, W., Couoh-Cardel, S., ... Kane, P. M. (2014). The signaling lipid PI(3,5)P2 stabilizes V1-Vo sector interactions and activates the VATPase. Molecular Biology of the Cell, 25(8), 1251-1262. https://doi.org/10.1091/mbc.E13-10-0563

Li, Y., Xu, M., Ding, X., Yan, C., Song, Z., Chen, L., .. Yang, C. (2016). Protein kinase C controls lysosome biogenesis independently of mTORC1. Nature Cell Biology, 18(10), 1065-1077. https://doi.org/10.1038/ncb3407

Liggins, M. C., Flesher, J. L., Jahid, S., Vasudeva, P., Eby, V., Takasuga, S., ... Ganesan, A. K. (2018). PIKfyve regulates melanosome biogenesis. PLoS Genetics, 14(3), e1007290. https://doi.org/10.1371/journal.pgen.1007290

Machesky, L. M., \& Insall, R. H. (1998). Scar1 and the related Wiskott-Aldrich syndrome protein, WASP, regulate the actin cytoskeleton through the Arp2/3 complex. Current Biology, 8(25), 1347-1356. https://doi.org/10.1016/S0960-9822(98)00015-3

Mao, Y., Yamaga, M., Zhu, X., Wei, Y., Sun, H.-Q., Wang, J., ... Yin, H. L. (2009). Essential and unique roles of PIP5K- $\gamma$ and $-\alpha$ in Fc $\gamma$ receptor-mediated phagocytosis. The Journal of Cell Biology, 184(2), 281-296. https://doi.org/10.1083/jcb.200806121

Marchand, B., Arsenault, D., Raymond-Fleury, A., Boisvert, F. M., \& Boucher, M. J. (2015). Glycogen synthase kinase-3 (GSK3) inhibition induces prosurvival autophagic signals in human pancreatic cancer cells. Journal of Biological Chemistry, 290(9), 5592-5605. https://doi.org/10.1074/jbc.M114.616714

Martina, J. A., Diab, H. I., Brady, O. A., \& Puertollano, R. (2016). TFEB and TFE3 are novel components of the integrated stress response. The EMBO Journal, 35(5), 479-495. https://doi.org/10.15252/embj.201593428

Martina, J. A., Diab, H. I., Lishu, L., Jeong-A, L., Patange, S., Raben, N., \& Puertollano, R. (2014). The nutrient-responsive transcription factor TFE3 promotes autophagy, lysosomal biogenesis, and clearance of cellular debris. Science Signaling, 7(309), ra9-ra9. https://doi.org/10.1126/scisignal.2004754

Martina, J. A., \& Puertollano, R. (2013). Rag GTPases mediate amino acid-dependent recruitment of TFEB and MITF to lysosomes. Journal of Cell Biology, 200(4), 475-491. https://doi.org/10.1083/jcb.201209135

Martina, J. A., \& Puertollano, R. (2018). Protein phosphatase 2A stimulates activation of TFEB and TFE3 transcription factors in response to oxidative stress. The Journal of Biological Chemistry, jbc.RA118.003471. https://doi.org/10.1074/jbc.RA118.003471

Martinez, F. O., \& Gordon, S. (2014). The M1 and M2 paradigm of macrophage activation: time for reassessment. F1000Prime Reports, 6, 13. https://doi.org/10.12703/P6-13

Massari, M. E., \& Murre, C. (2000). Helix-Loop-Helix Proteins: Regulators of Transcription in Eucaryotic Organisms. Molecular and Cellular Biology, 20(2), 429-440. 
https://doi.org/10.1128/MCB.20.2.429-440.2000

Mathews, P. M., Martinie, J. B., \& Fambrough, D. M. (1992). The pathway and targeting signal for delivery of the integral membrane glycoprotein LEP100 to lysosomes. Journal of Cell Biology, 118(5), 1027-1040. https://doi.org/10.1083/jcb.118.5.1027

May, R. C., Caron, E., Hall, A., \& Machesky, L. M. (2000). Involvement of the Arp2/3 complex in phagocytosis mediated by FcyR or CR3. Nature Cell Biology, 2(4), 246-248. https://doi.org/10.1038/35008673

McBride, H. M., Rybin, V., Murphy, C., Giner, A., Teasdale, R., \& Zerial, M. (1999). Oligomeric complexes link Rab5 effectors with NSF and drive membrane fusion via interactions between EEA1 and syntaxin 13. Cell, 98(3), 377-386. https://doi.org/10.1016/S0092-8674(00)81966-2

Medina, D. L., Di Paola, S., Peluso, I., Armani, A., De Stefani, D., Venditti, R., ... Ballabio, A. (2015). Lysosomal calcium signalling regulates autophagy through calcineurin and TFEB. Nature Cell Biology, 17(3), 288-299. https://doi.org/10.1038/ncb3114

Medina, D. L., Fraldi, A., Bouche, V., Annunziata, F., Mansueto, G., Spampanato, C., ... Ballabio, A. (2011). Transcriptional activation of lysosomal exocytosis promotes cellular clearance. Developmental Cell, 21(3), 421-430. https://doi.org/10.1016/j.devcel.2011.07.016

Miller, A. J., Levy, C., Davis, I. J., Razin, E., \& Fisher, D. E. (2005). Sumoylation of MITF and its related family members TFE3 and TFEB. Journal of Biological Chemistry, 280(1), 146155. https://doi.org/10.1074/jbc.M411757200

Milne, S. B., Ivanova, P. T., DeCamp, D., Hsueh, R. C., \& Brown, H. A. (2005). A targeted mass spectrometric analysis of phosphatidylinositol phosphate species. Journal of Lipid Research, 46(8), 1796-1802. https://doi.org/10.1194/jlr.D500010-JLR200

Minogue, S. (2006). Phosphatidylinositol 4-kinase is required for endosomal trafficking and degradation of the EGF receptor. Journal of Cell Science, 119(3), 571-581. https://doi.org/10.1242/jcs.02752

Miyanishi, M., Tada, K., Koike, M., Uchiyama, Y., Kitamura, T., \& Nagata, S. (2007). Identification of Tim4 as a phosphatidylserine receptor. Nature, 450(7168), 435-439. https://doi.org/10.1038/nature06307

Monks, J., Rosner, D., Geske, F. J., Lehman, L., Hanson, L., Neville, M. C., \& Fadok, V. A. (2005). Epithelial cells as phagocytes: Apoptotic epithelial cells are engulfed by mammary alveolar epithelial cells and repress inflammatory mediator release. Cell Death and Differentiation, 12(2), 107-114. https://doi.org/10.1038/sj.cdd.4401517

Moon, K. D., Post, C. B., Durden, D. L., Zhou, Q., De, P., Harrison, M. L., \& Geahlen, R. L. (2005). Molecular basis for a direct interaction between the Syk protein-tyrosine kinase and phosphoinositide 3-kinase. Journal of Biological Chemistry, 280(2), 1543-1551. https://doi.org/10.1074/jbc.M407805200

Mu, F. T., Callaghan, J. M., Steele-Mortimer, O., Stenmark, H., Parton, R. G., Campbell, P. L., 
... Toh, B. H. (1995). EEA1, an early endosome-associated protein. EEA1 is a conserved $\alpha-$ helical peripheral membrane protein flanked by cysteine "fingers" and contains a calmodulin-binding IQ motif. Journal of Biological Chemistry, 270(22), 13503-13511. https://doi.org/10.1074/jbc.270.22.13503

Najibi, M., Labed, S. A., Visvikis, O., \& Irazoqui, J. E. (2016). An Evolutionarily Conserved PLC-PKD-TFEB Pathway for Host Defense. Cell Reports, 15(8), 1728-1742. https://doi.org/10.1016/j.celrep.2016.04.052

Naufer, A., Hipolito, V. E. B., Ganesan, S., Prashar, A., Zaremberg, V., Botelho, R. J., \& Terebiznik, M. R. (2018). pH of endophagosomes controls association of their membranes with Vps34 and PtdIns(3)P levels. Journal of Cell Biology, 217(1), 329-346. https://doi.org/10.1083/jcb.201702179

Nezich, C. L., Wang, C., Fogel, A. I., \& Youle, R. J. (2015). MiT/TFE transcription factors are activated during mitophagy downstream of Parkin and Atg5. Journal of Cell Biology, 210(3), 435-450. https://doi.org/10.1083/jcb.201501002

Nixon, R. A. (2013). The role of autophagy in neurodegenerative disease. Nature Medicine, 19(8), 983-997. https://doi.org/10.1038/nm.3232

Nordmann, M., Cabrera, M., Perz, A., Bröcker, C., Ostrowicz, C., Engelbrecht-Vandré, S., \& Ungermann, C. (2010). The Mon1-Ccz1 complex is the GEF of the late endosomal Rab7 homolog Ypt7. Current Biology, 20(18), 1654-1659. https://doi.org/10.1016/j.cub.2010.08.002

O’Connell, R. M., Rao, D. S., \& Baltimore, D. (2012). microRNA Regulation of Inflammatory Responses. Annual Review of Immunology, 30(1), 295-312. https://doi.org/10.1146/annurev-immunol-020711-075013

Odorizzi, G., Babst, M., \& Emr, S. D. (1998). Fab1p Ptdlns(3)P 5-kinase function essential for protein sorting in the multivesicular body. Cell, 95(6), 847-858. https://doi.org/10.1016/S0092-8674(00)81707-9

Ohno, H., Stewart, J., Fournier, M. C., Bosshart, H., Rhee, I., Miyatake, S., ... Bonifacino, J. S. (1995). Interaction of tyrosine-based sorting signals with clathrin-associated proteins. Science, 269(5232), 1872-1875. https://doi.org/10.1126/science.7569928

Oppelt, A., Haugsten, E. M., Zech, T., Danielsen, H. E., Sveen, A., Lobert, V. H., ... Wesche, J. (2014). PIKfyve, MTMR3 and their product PtdIns5 $P$ regulate cancer cell migration and invasion through activation of Rac1. Biochemical Journal, 461(3), 383-390. https://doi.org/10.1042/BJ20140132

Ouimet, M., Koster, S., Sakowski, E., Ramkhelawon, B., Van Solingen, C., Oldebeken, S., ... Moore, K. J. (2016). Mycobacterium tuberculosis induces the MIR-33 locus to reprogram autophagy and host lipid metabolism. Nature Immunology, 17(6), 677-686. https://doi.org/10.1038/ni.3434

Palmieri, M., Impey, S., Kang, H., di Ronza, A., Pelz, C., Sardiello, M., \& Ballabio, A. (2011). Characterization of the CLEAR network reveals an integrated control of cellular clearance 
pathways. Human Molecular Genetics, 20(19), 3852-3866.

https://doi.org/10.1093/hmg/ddr306

Palmieri, M., Pal, R., Nelvagal, H. R., Lotfi, P., Stinnett, G. R., Seymour, M. L., ... Sardiello, M. (2017). MTORC1-independent TFEB activation via Akt inhibition promotes cellular clearance in neurodegenerative storage diseases. Nature Communications, 8, 14338. https://doi.org/10.1038/ncomms14338

Pastore, N., Brady, O. A., Diab, H. I., Martina, J. A., Sun, L., Huynh, T., ... Puertollano, R. (2016). TFEB and TFE3 cooperate in the regulation of the innate immune response in activated macrophages. Autophagy, 12(8), 1240-1258. https://doi.org/10.1080/15548627.2016.1179405

Patel, P., \& Woodgett, J. R. (2017). Glycogen Synthase Kinase 3: A Kinase for All Pathways? Current Topics in Developmental Biology, 123, 277-302. https://doi.org/10.1016/bs.ctdb.2016.11.011

Pauker, M. H., Reicher, B., Joseph, N., Wortzel, I., Jakubowicz, S., Noy, E., ... Barda-Saad, M. (2014). WASp family verprolin-homologous protein-2 (WAVE2) and Wiskott-Aldrich Syndrome Protein (WASp) engage in distinct downstream signaling interactions at the T cell antigen receptor site. Journal of Biological Chemistry, $289(50)$, 34503-34519. https://doi.org/10.1074/jbc.M114.591685

Payne, D. M., Rossomando, A. J., Martino, P., Erickson, A. K., Her, J. H., Shabanowitz, J., ... Sturgill, T. W. (1991). Identification of the regulatory phosphorylation sites in pp42/mitogen-activated protein kinase (MAP kinase). The EMBO Journal, 10(4), 885-92. https://doi.org/10.1002/j.1460-2075.1995.tb00212.x

Pearson, R. B., Dennis, P. B., Han, J. W., Williamson, N. A., Kozma, S. C., Wettenhall, R. E., \& Thomas, G. (1995). The principal target of rapamycin-induced p70s6k inactivation is a novel phosphorylation site within a conserved hydrophobic domain. The EMBO Journal, 14(21), 5279-87. https://doi.org/10.1002/j.1460-2075.1991.tb08021.x

Pei, G., Repnik, U., Griffiths, G., \& Gutierrez, M. G. (2014). Identification of an immuneregulated phagosomal Rab cascade in macrophages. Journal of Cell Science, 127(9), 20712082. https://doi.org/10.1242/jcs. 144923

Peiser, L., De Winther, M. P. J., Makepeace, K., Hollinshead, M., Coull, P., Plested, J., ... Gordon, S. (2002). The class A macrophage scavenger receptor is a major pattern recognition receptor for Neisseria meningitidis which is independent of lipopolysaccharide and not required for secretory responses. Infection and Immunity, 70(10), 5346-5354. https://doi.org/10.1128/IAI.70.10.5346-5354.2002

Perera, R. M., Stoykova, S., Nicolay, B. N., Ross, K. N., Fitamant, J., Boukhali, M., ... Bardeesy, N. (2015). Transcriptional control of autophagy-lysosome function drives pancreatic cancer metabolism. Nature, 524(7565), 361-365. https://doi.org/10.1038/nature14587

Ploper, D., Taelman, V. F., Robert, L., Perez, B. S., Titz, B., Chen, H.-W., ... De Robertis, E. M. (2015). MITF drives endolysosomal biogenesis and potentiates Wnt signaling in melanoma 
cells. Proceedings of the National Academy of Sciences, 112(5), E420-E429.

https://doi.org/10.1073/pnas.1424576112

Pogenberg, V., Ogmundsdóttir, M. H., Bergsteinsdóttir, K., Schepsky, A., Phung, B., Deineko, V., ... Wilmanns, M. (2012). Restricted leucine zipper dimerization and specificity of DNA recognition of the melanocyte master regulator MITF. Genes \& Development, 26(23), 2647-58. https://doi.org/10.1101/gad.198192.112

Poirier, M. G., Eroglu, S., \& Marko, J. F. (2002). The bending rigidity of mitotic chromosomes. Molecular Biology of the Cell, 13(6), 2170-2179. https://doi.org/10.1091/mbc.01

Polito, V. a, Li, H., Martini-Stoica, H., Wang, B., Yang, L., Xu, Y., ... Zheng, H. (2014). Selective clearance of aberrant tau proteins and rescue of neurotoxicity by transcription factor EB. EMBO Molecular Medicine, 6(9), 1142-60. https://doi.org/10.15252/emmm.201303671

Porstmann, T., Santos, C. R., Griffiths, B., Cully, M., Wu, M., Leevers, S., ... Schulze, A. (2008). SREBP Activity Is Regulated by mTORC1 and Contributes to Akt-Dependent Cell Growth. Cell Metabolism, 8(3), 224-236. https://doi.org/10.1016/j.cmet.2008.07.007

Poteryaev, D., Datta, S., Ackema, K., Zerial, M., \& Spang, A. (2010). Identification of the Switch in Early-to-Late Endosome Transition. Cell, 141(3), 497-508. https://doi.org/10.1016/j.cell.2010.03.011

Proikas-Cezanne, T., Takacs, Z., Donnes, P., \& Kohlbacher, O. (2015). WIPI proteins: essential PtdIns3P effectors at the nascent autophagosome. Journal of Cell Science, 128(2), 207-217. https://doi.org/10.1242/jcs.146258

Puertollano, R., Ferguson, S. M., Brugarolas, J., \& Ballabio, A. (2018). The complex relationship between TFEB transcription factor phosphorylation and subcellular localization. The EMBO Journal, 37(11), e98804. https://doi.org/10.15252/embj.201798804

Rabinovitch, M. (1995). Professional and non-professional phagocytes: an introduction. Trends in Cell Biology, 5(3), 85-87. https://doi.org/10.1016/S0962-8924(00)88955-2

Rebsamen, M., Pochini, L., Stasyk, T., De Araújo, M. E. G., Galluccio, M., Kandasamy, R. K., ... Superti-Furga, G. (2015). SLC38A9 is a component of the lysosomal amino acid sensing machinery that controls mTORC1. Nature, 519(7544), 477-481. https://doi.org/10.1038/nature14107

Rink, J., Ghigo, E., Kalaidzidis, Y., \& Zerial, M. (2005). Rab conversion as a mechanism of progression from early to late endosomes. Cell, 122(5), 735-749. https://doi.org/10.1016/j.cell.2005.06.043

Roczniak-Ferguson, A., Petit, C. S., Froehlich, F., Qian, S., Ky, J., Angarola, B., ... Ferguson, S. M. (2012). The transcription factor TFEB links mTORC1 signaling to transcriptional control of lysosome homeostasis. Science Signaling, 5(228), ra42. https://doi.org/10.1126/scisignal.2002790

Rudge, S. A. (2003). Vacuole Size Control: Regulation of PtdIns(3,5)P2 Levels by the Vacuoleassociated Vac14-Fig4 Complex, a PtdIns(3,5)P2-specific Phosphatase. Molecular Biology 
of the Cell, 15(1), 24-36. https://doi.org/10.1091/mbc.E03-05-0297

Rutherford, A. C. (2006). The mammalian phosphatidylinositol 3-phosphate 5-kinase (PIKfyve) regulates endosome-to-TGN retrograde transport. Journal of Cell Science, 119(19), 39443957. https://doi.org/10.1242/jcs.03153

Saha, S., Panigrahi, D. P., Patil, S., \& Bhutia, S. K. (2018). Autophagy in health and disease: A comprehensive review. Biomedicine and Pharmacotherapy, 104, 485-495. https://doi.org/10.1016/j.biopha.2018.05.007

Sakurai, C., Hashimoto, H., Nakanishi, H., Arai, S., Wada, Y., Sun-Wada, G.-H., ... Hatsuzawa, K. (2012). SNAP-23 regulates phagosome formation and maturation in macrophages. Molecular Biology of the Cell, 23(24), 4849-4863. https://doi.org/10.1091/mbc.E12-010069

Samie, M., \& Cresswell, P. (2015). The transcription factor TFEB acts as a molecular switch that regulates exogenous antigen-presentation pathways. Nature Immunology, 16(7), 729-736. https://doi.org/10.1038/ni.3196

Sancak, Y., Bar-Peled, L., Zoncu, R., Markhard, A. L., Nada, S., \& Sabatini, D. M. (2010). Ragulator-rag complex targets mTORC1 to the lysosomal surface and is necessary for its activation by amino acids. Cell, 141(2), 290-303. https://doi.org/10.1016/j.cell.2010.02.024

Sancak, Y., Peterson, T. R., Shaul, Y. D., Lindquist, R. A., Thoreen, C. C., Bar-Peled, L., \& Sabatini, D. M. (2008). The rag GTPases bind raptor and mediate amino acid signaling to mTORC1. Science, 320(5882), 1496-1501. https://doi.org/10.1126/science.1157535

Sardiello, M., Palmieri, M., Ronza, A. Di, Medina, D. L., Valenza, M., Gennarino, V. A., ... Ballabio, A. (2009). A gene network regulating lysosomal biogenesis and function. Science, 325(5939), 473-477. https://doi.org/10.1126/science.1174447

Sarkar, S., Davies, J. E., Huang, Z., Tunnacliffe, A., \& Rubinsztein, D. C. (2007). Trehalose, a novel mTOR-independent autophagy enhancer, accelerates the clearance of mutant huntingtin and $\alpha$-synuclein. Journal of Biological Chemistry, 282(8), 5641-5652. https://doi.org/10.1074/jbc.M609532200

Sasaki, T., Takasuga, S., Sasaki, J., Kofuji, S., Eguchi, S., Yamazaki, M., \& Suzuki, A. (2009). Mammalian phosphoinositide kinases and phosphatases. Progress in Lipid Research, 48(6), 307-343. https://doi.org/10.1016/j.plipres.2009.06.001

Saveanu, L., \& Lotersztajn, S. (2016). New pieces in the complex puzzle of aberrant vacuolation. Focus on "Active vacuolar H + ATPase and functional cycle of Rab5 are required for the vacuolation defect triggered by PtdIns(3,5)P 2 loss under PIKfyve or Vps34 defic. American Journal of Physiology - Cell Physiology, 311(3), C363-C365. https://doi.org/10.1152/ajpcell.00215.2016

Saxton, R. A., Knockenhauer, K. E., Wolfson, R. L., Chantranupong, L., Pacold, M. E., Wang, T., ... Sabatini, D. M. (2016). Structural basis for leucine sensing by the Sestrin2-mTORC1 pathway. Science, 351(6268), 53-58. https://doi.org/10.1126/science.aad2087

Saxton, R. A., \& Sabatini, D. M. (2017). mTOR Signaling in Growth, Metabolism, and Disease. 
Cell, 168(6), 960-976. https://doi.org/10.1016/j.cell.2017.02.004

Sbrissa, D., Ikonomov, O. C., Fenner, H., \& Shisheva, A. (2008). ArPIKfyve Homomeric and Heteromeric Interactions Scaffold PIKfyve and Sac3 in a Complex to Promote PIKfyve Activity and Functionality. Journal of Molecular Biology, 384(4), 766-779. https://doi.org/10.1016/j.jmb.2008.10.009

Sbrissa, D., Ikonomov, O. C., \& Shisheva, A. (2002). Phosphatidylinositol 3-phosphateinteracting domains in PIKfyve. Binding specificity and role in PIKfyve endomembrane localization. Journal of Biological Chemistry, 277(8), 6073-6079. https://doi.org/10.1074/jbc.M110194200

Scacioc, A., Schmidt, C., Hofmann, T., Urlaub, H., Kühnel, K., \& Pérez-Lara, Á. (2017). Structure based biophysical characterization of the PROPPIN Atg18 shows Atg18 oligomerization upon membrane binding. Scientific Reports, 7(1), 14008. https://doi.org/10.1038/s41598-017-14337-5

Schlam, D., Bagshaw, R. D., Freeman, S. A., Collins, R. F., Pawson, T., Fairn, G. D., \& Grinstein, S. (2015). Phosphoinositide 3-kinase enables phagocytosis of large particles by terminating actin assembly through Rac/Cdc42 GTPase-activating proteins. Nature Communications, 6, 8623. https://doi.org/10.1038/ncomms9623

Schmid, A. C., Wise, H. M., Mitchell, C. A., Nussbaum, R., \& Woscholski, R. (2004). Type II phosphoinositide 5-phosphatases have unique sensitivities towards fatty acid composition and head group phosphorylation. FEBS Letters, 576(1-2), 9-13. https://doi.org/10.1016/j.febslet.2004.08.052

Schneider-Poetsch, T., Ju, J., Eyler, D. E., Dang, Y., Bhat, S., Merrick, W. C., ... Liu, J. O. (2010). Inhibition of eukaryotic translation elongation by cycloheximide and lactimidomycin. Nature Chemical Biology, 6(3), 209-217. https://doi.org/10.1038/nchembio.304

Schröder, B. A., Wrocklage, C., Hasilik, A., \& Saftig, P. (2010). The proteome of lysosomes. Proteomics, 10(22), 4053-4076. https://doi.org/10.1002/pmic.201000196

Schu, P. V., Takegawa, K., Fry, M. J., Stack, J. H., Waterfield, M. D., \& Emr, S. D. (1993). Phosphatidylinositol 3-kinase encoded by yeast VPS34 gene essential for protein sorting. Science, 260(5104), 88-91. https://doi.org/10.1126/science.8385367

Schulze, U., Vollenbröker, B., Kühnl, A., Granado, D., Bayraktar, S., Rescher, U., ... Weide, T. (2017). Cellular vacuolization caused by overexpression of the PIKfyve-binding deficient Vac14L156Ris rescued by starvation and inhibition of vacuolar-ATPase. Biochimica et Biophysica Acta - Molecular Cell Research, 1864(5), 749-759. https://doi.org/10.1016/j.bbamcr.2017.02.012

Scott, C. C., Dobson, W., Botelho, R. J., Coady-Osberg, N., Chavrier, P., Knecht, D. A., ... Grinstein, S. (2005). Phosphatidylinositol-4, 5-bisphosphate hydrolysis directs actin remodeling during phagocytosis. Journal of Cell Biology, 169(1), 139-149. https://doi.org/10.1083/jcb.200412162 
Seals, D. F., Eitzen, G., Margolis, N., Wickner, W. T., \& Price, A. (2000). A Ypt/Rab effector complex containing the Sec1 homolog Vps33p is required for homotypic vacuole fusion. Proceedings of the National Academy of Sciences, 97(17), 9402-9407. https://doi.org/10.1073/pnas.97.17.9402

Seaman, M. N. J., McCaffery, J. M., \& Emr, S. D. (1998). A membrane coat complex essential for endosome-to-Golgi retrograde transport in yeast. Journal of Cell Biology, 142(3), 665681. https://doi.org/10.1083/jcb.142.3.665

Settembre, C., Di Malta, C., Polito, V. A., Arencibia, M. G., Vetrini, F., Erdin, S., ... Ballabio, A. (2011). TFEB links autophagy to lysosomal biogenesis. Science, 332(6036), 1429-1433. https://doi.org/10.1126/science. 1204592

Settembre, C., Zoncu, R., Medina, D. L., Vetrini, F., Erdin, S., Erdin, S., ... Ballabio, A. (2012). A lysosome-to-nucleus signalling mechanism senses and regulates the lysosome via mTOR and TFEB. EMBO Journal, 31(5), 1095-1108. https://doi.org/10.1038/emboj.2012.32

Shen, J., Yu, W. M., Brotto, M., Scherman, J. A., Guo, C., Stoddard, C., .. Qu, C. K. (2009). Deficiency of MIP/MTMR14 phosphatase induces a muscle disorder by disrupting $\mathrm{Ca} 2+$ homeostasis. Nature Cell Biology, 11(6), 769-776. https://doi.org/10.1038/ncb1884

Shulga, Y. V., Anderson, R. A., Topham, M. K., \& Epand, R. M. (2012). Phosphatidylinositol-4phosphate 5-kinase isoforms exhibit acyl chain selectivity for both substrate and lipid activator. Journal of Biological Chemistry, 287(43), 35953-35963. https://doi.org/10.1074/jbc.M112.370155

Sigola, L. B., Fuentes, A. L., Millis, L. M., Vapenik, J., \& Murira, A. (2016). Effects of Toll-like receptor ligands on RAW 264.7 macrophage morphology and zymosan phagocytosis. Tissue and Cell, 48(4), 389-396. https://doi.org/10.1016/j.tice.2016.04.002

Simonsen, A., Gaullier, J. M., D’Arrigo, A., \& Stenmark, H. (1999). The Rab5 effector EEA1 interacts directly with syntaxin-6. Journal of Biological Chemistry, 274(41), 28857-28860. https://doi.org/10.1074/jbc.274.41.28857

Slade, L., \& Pulinilkunnil, T. (2017). The MiTF/TFE Family of Transcription Factors: Master Regulators of Organelle Signaling, Metabolism, and Stress Adaptation. Molecular Cancer Research, 15(12), 1637-1643. https://doi.org/10.1158/1541-7786.MCR-17-0320

Sobota, A., Strzelecka-Kiliszek, A., G adkowska, E., Yoshida, K., Mrozinska, K., \& Kwiatkowska, K. (2005). Binding of IgG-Opsonized Particles to Fc R Is an Active Stage of Phagocytosis That Involves Receptor Clustering and Phosphorylation. The Journal of Immunology, 175(7), 4450-4457. https://doi.org/10.4049/jimmunol.175.7.4450

Spampanato, C., Feeney, E., Li, L., Cardone, M., Lim, J. A., Annunziata, F., ... Raben, N. (2013). Transcription factor EB (TFEB) is a new therapeutic target for Pompe disease. EMBO Molecular Medicine, 5(5), 691-706. https://doi.org/10.1002/emmm.201202176

Stambolic, V., \& Woodgett, J. R. (1994). Mitogen inactivation of glycogen synthase kinase-3 beta in intact cells via serine 9 phosphorylation. The Biochemical Journal, 303 ( Pt 3, 7014. https://doi.org/10.1042/BJ3030701 
Steingrímsson, E., Copeland, N. G., \& Jenkins, N. A. (2004). Melanocytes and the Microphthalmia Transcription Factor Network. Annual Review of Genetics, 38(1), 365-411. https://doi.org/10.1146/annurev.genet.38.072902.092717

Stenmark, H., Aasland, R., Toh, B. H., \& D’Arrigo, A. (1996). Endosomal localization of the autoantigen EEA1 is mediated by a zinc- binding FYVE finger. Journal of Biological Chemistry, 271(39), 24048-24054. https://doi.org/10.1074/jbc.271.39.24048

Stenmark, H., Vitale, G., Ullrich, O., \& Zerial, M. (1995). Rabaptin-5 is a direct effector of the small GTPase Rab5 in endocytic membrane fusion. Cell, 83(3), 423-432. https://doi.org/10.1016/0092-8674(95)90120-5

Sun-Wada, G.-H., Tabata, H., Kawamura, N., Aoyama, M., \& Wada, Y. (2009). Direct recruitment of H+-ATPase from lysosomes for phagosomal acidification. Journal of Cell Science, 122(14), 2504-2513. https://doi.org/10.1242/jcs.050443

Sutherland, C., \& Cohen, P. (1994). The $\alpha$-isoform of glycogen synthase kinase-3 from rabbit skeletal muscle is inactivated by p70 S6 kinase or MAP kinase-activated protein kinase-1 in vitro. FEBS Letters, 338(1), 37-42. https://doi.org/10.1016/0014-5793(94)80112-6

Sutherland, C., Leighton, I. A., \& Cohen, P. (1993). Inactivation of glycogen synthase kinase-3fl by phosphorylation: new kinase connections in insulin and growth-factor signalling. Biochem. J, 296, 15-19. https://doi.org/10.1042/bj2960015

Suzuki, T., Kono, H., Hirose, N., Okada, M., Yamamoto, T., Yamamoto, K., \& Honda, Z. -i. (2000). Differential Involvement of Src Family Kinases in Fc Receptor-Mediated Phagocytosis. The Journal of Immunology, 165(1), 473-482. https://doi.org/10.4049/jimmunol.165.1.473

Swanson, J. A., \& Baer, S. C. (1995). Phagocytosis by zippers and triggers. Trends in Cell Biology, 5(3), 89-93. https://doi.org/10.1016/S0962-8924(00)88956-4

Szondy, Z., Sarang, Z., Kiss, B., Garabuczi, É., \& Köröskényi, K. (2017). Anti-inflammatory mechanisms triggered by apoptotic cells during their clearance. Frontiers in Immunology, 8(AUG), 909. https://doi.org/10.3389/fimmu.2017.00909

Thoreen, C. C., Kang, S. A., Chang, J. W., Liu, Q., Zhang, J., Gao, Y., .. Gray, N. S. (2009). An ATP-competitive mammalian target of rapamycin inhibitor reveals rapamycin-resistant functions of mTORC1. Journal of Biological Chemistry, 284(12), 8023-8032. https://doi.org/10.1074/jbc.M900301200

Tomasevic, N., Jia, Z., Russell, A., Fujii, T., Hartman, J. J., Clancy, S., ... Sakowicz, R. (2007). Differential regulation of WASP and N-WASP by Cdc42, Rac1, Nck, and PI(4,5)P2. Biochemistry, 46(11), 3494-3502. https://doi.org/10.1021/bi062152y

Tridandapani, S., Lyden, T. W., Smith, J. L., Carter, J. E., Coggeshall, K. M., \& Anderson, C. L. (2000). The adapter protein LAT enhances Fc $\gamma$ receptor-mediated signal transduction in myeloid cells. Journal of Biological Chemistry, 275(27), 20480-20487. https://doi.org/10.1074/jbc.M909462199

Tse, S. M. L., Furuya, W., Gold, E., Schreiber, A. D., Sandvig, K., Inman, R. D., \& Grinstein, S. 
(2003). Differential role of actin, clathrin, and dynamin in Fcy receptor-mediated endocytosis and phagocytosis. Journal of Biological Chemistry, 278(5), 3331-3338. https://doi.org/10.1074/jbc.M207966200

Vega-Rubin-de-Celis, S., Peña-Llopis, S., Konda, M., \& Brugarolas, J. (2017). Multistep regulation of TFEB by MTORC1. Autophagy, 13(3), 464-472. https://doi.org/10.1080/15548627.2016.1271514

Via, L. E., Deretic, D., Ulmer, R. J., Hibler, N. S., Huber, L. A., \& Deretic, V. (1997). Arrest of mycobacterial phagosome maturation is caused by a block in vesicle fusion between stages controlled by rab5 and rab7. Journal of Biological Chemistry, 272(20), 13326-13331. https://doi.org/10.1074/jbc.272.20.13326

Vidarsson, G., Dekkers, G., \& Rispens, T. (2014). IgG subclasses and allotypes: From structure to effector functions. Frontiers in Immunology, 5(OCT), 520. https://doi.org/10.3389/fimmu.2014.00520

Vieira, O. V., Botelho, R. J., Rameh, L., Brachmann, S. M., Matsuo, T., Davidson, H. W., ... Grinstein, S. (2001). Distinct roles of class I and class III phosphatidylinositol 3-kinases in phagosome formation and maturation. Journal of Cell Biology, 155(1), 19-25. https://doi.org/10.1083/jcb.200107069

Vieira, O. V., Bucci, C., Harrison, R. E., Trimble, W. S., Lanzetti, L., Gruenberg, J., ... Grinstein, S. (2003). Modulation of Rab5 and Rab7 Recruitment to Phagosomes by Phosphatidylinositol 3-Kinase. Molecular and Cellular Biology, 23(7), 2501-2514. https://doi.org/10.1128/MCB.23.7.2501-2514.2003

Visvikis, O., Ihuegbu, N., Labed, S. A., Luhachack, L. G., Alves, A. M. F., Wollenberg, A. C., ... Irazoqui, J. E. (2014). Innate host defense requires TFEB-mediated transcription of cytoprotective and antimicrobial genes. Immunity, 40(6), 896-909. https://doi.org/10.1016/j.immuni.2014.05.002

Vural, A., Al-Khodor, S., Cheung, G. Y. C., Shi, C.-S., Srinivasan, L., McQuiston, T. J., ... Kehrl, J. H. (2016). Activator of G-Protein Signaling 3-Induced Lysosomal Biogenesis Limits Macrophage Intracellular Bacterial Infection. The Journal of Immunology, 196(2), 846-856. https://doi.org/10.4049/jimmunol.1501595

Walker, D. M., Urbé, S., Dove, S. K., Tenza, D., Raposo, G., \& Clague, M. J. (2001). Characterization of MTMR3: An inositol lipid 3-phosphatase with novel substrate specificity. Current Biology, 11(20), 1600-1605. https://doi.org/10.1016/S09609822(01)00501-2

Walker, M. R., Lund, J., Thompson, K. M., \& Jefferis, R. (1989). Aglycosylation of human IgG1 and IgG3 monoclonal antibodies can eliminate recognition by human cells expressing Fc gamma RI and/or Fc gamma RII receptors. The Biochemical Journal, 259(2), 347-53. Retrieved from http://www.ncbi.nlm.nih.gov/pubmed/2524188

Wang, H., Sun, H.-Q., Zhu, X., Zhang, L., Albanesi, J., Levine, B., \& Yin, H. (2015). GABARAPs regulate PI4P-dependent autophagosome:lysosome fusion. Proceedings of the National Academy of Sciences, 112(22), 7015-7020. 
https://doi.org/10.1073/pnas.1507263112

Wang, S., Tsun, Z. Y., Wolfson, R. L., Shen, K., Wyant, G. A., Plovanich, M. E., ... Sabatini, D. M. (2015). Lysosomal amino acid transporter SLC38A9 signals arginine sufficiency to mTORC1. Science, 347(6218), 188-194. https://doi.org/10.1126/science.1257132

Wang, W., Gao, Q., Yang, M., Zhang, X., Yu, L., Lawas, M., ... Xu, H. (2015). Up-regulation of lysosomal TRPML1 channels is essential for lysosomal adaptation to nutrient starvation. Proceedings of the National Academy of Sciences, 112(11), E1373-E1381. https://doi.org/10.1073/pnas.1419669112

Wang, X., Zhang, X., Dong, X. P., Samie, M., Li, X., Cheng, X., ... Xu, H. (2012). TPC proteins are phosphoinositide- Activated sodium-selective ion channels in endosomes and lysosomes. Cell, 151(2), 372-383. https://doi.org/10.1016/j.cell.2012.08.036

Wang, Y. J., Wang, J., Sun, H. Q., Martinez, M., Sun, Y. X., Macia, E., ... Yin, H. L. (2003). Phosphatidylinositol 4 phosphate regulates targeting of clathrin adaptor AP-1 complexes to the Golgi. Cell, 114(3), 299-310. https://doi.org/10.1016/S0092-8674(03)00603-2

Wassmer, T., Attar, N., Bujny, M. V., Oakley, J., Traer, C. J., \& Cullen, P. J. (2006). A loss-offunction screen reveals SNX5 and SNX6 as potential components of the mammalian retromer. Journal of Cell Science, 120(1), 45-54. https://doi.org/10.1242/jcs.03302

Wilson, Z. N., Scott, A. L., Dowell, R. D., \& Odorizzi, G. (2018). PI(3,5)P 2 controls vacuole potassium transport to support cellular osmoregulation. Molecular Biology of the Cell, 29(13), 1718-1731. https://doi.org/10.1091/mbc.E18-01-0015

Wolfson, R. L., Chantranupong, L., Saxton, R. A., Shen, K., Scaria, S. M., Cantor, J. R., \& Sabatini, D. M. (2016). Sestrin2 is a leucine sensor for the mTORC1 pathway. Science, 351(6268), 43-48. https://doi.org/10.1126/science.aab2674

Wong, C. O., Gregory, S., Hu, H., Chao, Y., Sepúlveda, V. E., He, Y., ... Venkatachalam, K. (2017). Lysosomal Degradation Is Required for Sustained Phagocytosis of Bacteria by Macrophages. Cell Host and Microbe, 21(6), 719-730.e6. https://doi.org/10.1016/j.chom.2017.05.002

Xiao, Q., Yan, P., Ma, X., Liu, H., Perez, R., Zhu, A., ... Lee, J.-M. (2014). Enhancing Astrocytic Lysosome Biogenesis Facilitates A Clearance and Attenuates Amyloid Plaque Pathogenesis. Journal of Neuroscience, 34(29), 9607-9620. https://doi.org/10.1523/JNEUROSCI.3788-13.2014

Xiong, J., \& Zhu, M. X. (2016). Regulation of lysosomal ion homeostasis by channels and transporters. Science China Life Sciences, 59(8), 777-791. https://doi.org/10.1007/s11427016-5090-x

Yamamoto, A., DeWald, D. B., Boronenkov, I. V., Anderson, R. A., Emr, S. D., \& Koshland, D. (1995). Novel PI(4)P 5-kinase homologue, Fab1p, essential for normal vacuole function and morphology in yeast. Molecular Biology of the Cell, 6(5), 525-539. https://doi.org/10.1091/mbc.6.5.525

Yarmolinsky, M. B., \& Haba, G. L. (1959). Inhibition By Puromycin of Amino Acid 
Incorporation Into Protein. Proceedings of the National Academy of Sciences of the United States of America, 45(12), 1721-1729. https://doi.org/10.1073/pnas.45.12.1721

Yasuda, S., Morishita, S., Fujita, A., Nanao, T., Wada, N., Waguri, S., .. Nakamura, T. (2016). Mon1-Ccz1 activates Rab7 only on late endosomes and dissociates from the lysosome in mammalian cells. Journal of Cell Science, 129(2), 329-340.

https://doi.org/10.1242/jcs.178095

Yu, J. W., \& Lemmon, M. A. (2001). All Phox Homology (PX) Domains from Saccharomyces cerevisiae Specifically Recognize Phosphatidylinositol 3-Phosphate. Journal of Biological Chemistry, 276(47), 44179-44184. https://doi.org/10.1074/jbc.M108811200

Zhang, J., Wang, J., Zhou, Z., Park, J.-E., Wang, L., Wu, S., ... Shen, H.-M. (2018). Importance of TFEB acetylation in control of its transcriptional activity and lysosomal function in response to histone deacetylase inhibitors. Autophagy, 14(6), 1-17. https://doi.org/10.1080/15548627.2018.1447290

Zhang, Y., Zolov, S. N., Chow, C. Y., Slutsky, S. G., Richardson, S. C., Piper, R. C., ... Weisman, L. S. (2007). Loss of Vac14, a regulator of the signaling lipid phosphatidylinositol 3,5-bisphosphate, results in neurodegeneration in mice. Proceedings of the National Academy of Sciences, 104(44), 17518-17523. https://doi.org/10.1073/pnas.0702275104

Zhang, Z., Zhang, T., Wang, S., Gong, Z., Tang, C., Chen, J., \& Ding, J. (2014). Molecular mechanism for Rabex-5 GEF activation by Rabaptin-5. ELife, 2014(3), e02687. https://doi.org/10.7554/eLife.02687.001

Zhao, G. Q., Zhao, Q., Zhou, X., Mattei, M. G., \& de Crombrugghe, B. (1993). TFEC, a basic helix-loop-helix protein, forms heterodimers with TFE3 and inhibits TFE3-dependent transcription activation. Molecular and Cellular Biology, 13(8), 4505-4512. https://doi.org/10.1128/MCB.13.8.4505

Zolov, S. N., Bridges, D., Zhang, Y., Lee, W.-W., Riehle, E., Verma, R., ... Weisman, L. S. (2012). In vivo, Pikfyve generates PI(3,5)P2, which serves as both a signaling lipid and the major precursor for PI5P. Proceedings of the National Academy of Sciences, 109(43), 17472-17477. https://doi.org/10.1073/pnas.1203106109

Zoncu, R., Bar-Peled, L., Efeyan, A., Wang, S., Sancak, Y., \& Sabatini, D. M. (2011). mTORC1 senses lysosomal amino acids through an inside-out mechanism that requires the vacuolar H+-ATPase. Science, 334(6056), 678-683. https://doi.org/10.1126/science.1207056 